\title{
58. GEOCHEMISTRY OF IGNEOUS ROCKS FROM LEGS 127 AND 128, SEA OF JAPAN ${ }^{1}$
}

\author{
James F. Allan ${ }^{2,3}$ and Michael P. Gorton ${ }^{4}$
}

\begin{abstract}
Legs 127 and 128 of the Ocean Drilling Program cored basement samples from two sites in the Yamato Basin (Sites 794 and 797) and one site in the Japan Basin (Site 795) of the Japan Sea. These samples represent sills and lava flows erupted or shallowly intruded in a marine environment during backarc extension and spreading in the middle Miocene. In this paper, we describe the geochemical characteristics of these igneous units using 52 new instrumental neutron activation analyses (INAA), 8 new X-ray fluorescence (XRF) analyses, and previous shipboard XRF analyses.

The sills intruded into soft sediment at Sites 794 and 797 were subject to extensive hydrothermal activity, estimated at $<230^{\circ} \mathrm{C}$ under subgreenschist facies conditions, which heavily to totally altered the fine-grained unit margins and moderately to heavily altered the coarse-grained unit interiors. Diagenesis further altered the composition of these igneous bodies and lava flows at Sites 794, 795, and 797, most intensely at unit margins. Our study of two well-sampled units shows that Mg, $\mathrm{Ca}, \mathrm{Sr}$, and the large-ion lithophile elements (LILE) mobilized during alteration, and that the concentrations of $\mathrm{Y}, \mathrm{Yb}$, and $\mathrm{Lu}$ decreased and $\mathrm{Ce}$ increased in the most severely altered samples. Nevertheless, our study shows that the rare-earth elements (REE) were relatively immobile in the majority of the samples, even where secondary mixed-layer clays comprised the great majority of the rock.

Fresher Yamato Basin samples are compositionally heterogenous tholeiitic basalts and dolerites. At Site 794 in the north-central portion of the basin, Units 1 to 5 (upper basement) comprise mildly light rare-earth element (LREE) enriched basalts and dolerites (chondrite-normalized La/Sm of 1.4-1.8), while the stratigraphically lower Units 6 to 9 are less enriched dolerites with $(\mathrm{La} / \mathrm{Sm})_{\mathrm{N}}$ of $0.7-1.3$. All Site 794 samples lack $\mathrm{Nb}$ and Ta depletions and LILE enrichments, lacking a strong subduction-related incompatible element geochemical signature. At Site 797 in the western margin of the basin, two stratigraphically-definable unit groups also occur. The upper nine units are incompatible-element depleted tholeiitic sills and flows with strong depletions of $\mathrm{Nb}$ and Ta relative to normal mid-ocean ridge basalt (N-MORB). The lower twelve sills represent LREE-enriched tholeiites (normalized $\mathrm{La} / \mathrm{Sm}$ ranges from 1.1 to 1.8 ), with distinctly higher LILE and high field-strength element (HFSE) contents. At Site 795 at the northern margin of the Japan Sea, three eruptive units consist of basaltic andesite to calc-alkaline basalt (normalized La/Sm of 1.1 to 1.5 ) containing moderate depletions of the HFSE relative to N-MORB. The LILE-depleted nature of these samples precludes their origin in a continental arc, indicating that they more likely erupted within a rifting oceanic arc system.

The heterogenous nature of the Japan Sea rocks indicate that they were derived at each site from multiple parental magmas generated from a compositionally heterogenous mantle source. Their chemistry is intermediate in character between arc basalts, MORB, and intraplate basalts, and implies little involvement of continental crust at any point in their genesis. Their flat chondrite-normalized, medium-to-heavy rare earth patterns indicate that the primary magmas which produced them last equilibrated with and segregated from spinel lherzolite at shallow depths $(<30 \mathrm{kbar})$. In strong contrast to their isotopic compositional arrays, subduction-related geochemical signatures are usually poorly defined. No basin-wide temporal or geographic systematics of rock chemistry may be confidently detailed; instead, the data show both intimate (site-specific) and widespread backarc mantle heterogeneity over a narrow ( $2 \mathrm{Ma}$ or so) range in time, with mantle heterogeneity most closely resembling a "plum-pudding" model.
\end{abstract}

\section{INTRODUCTION}

The purpose of Legs 127 and 128 of the Ocean Drilling Program (ODP) was to determine the basinal history of the Japan Sea. The Japan Sea, one of several marginal basins that lie at the western edge of the Pacific Ocean, is generally understood to have formed by means of the rifting of a continental volcanic arc in the mid- Tertiary (Tamaki, Pisciotto, Allan, et al., 1990; Tamaki, 1985; Celaya and McCabe, 1987; Hilde and Wageman, 1973; Karig, 1971, 1974; Lallemand and Jolivet, 1986; Shimazu et al., 1990). A primary goal of the leg was to determine the nature and age of the crust underlying this backarc basin and to determine its spreading history. The Japan Sea actually comprises two basins, the Yamato and Japan basins, separated by the rifted continental crust of the Yamato Rise (Fig. 1). Basement coring during

${ }^{1}$ Tamaki, K., Suyehiro, K., Allan, J., McWilliams, M., et al., 1992. Proc. ODP, Sci. Results, 127/128, Pt. 2: College Station, TX (Ocean Drilling Program).

${ }^{2}$ Ocean Drilling Program, Texas A\&M University, 1000 Discovery Drive, College Station, TX 77845, U.S.A. U.S.A.

Also at: Department Of Geology, Texas A\&M University, College Station, TX 77843 ,

${ }^{4}$ Department of Geology, University of Toronto, Toronto, ON M5S 1 A7 Canada the legs was focused at two sites within the Yamato Basin: one at the western basinal margin (Site 797), and one in the north central margin of the basin (Site 794). Basement was also cored at Site 795, at the northeastern margin of the Japan Basin (Fig. 1).

In this report, we present the initial results of new neutron activation analyses (INAA) and X-ray fluorescence analyses (XRF) of whole-rock powders from samples collected during Legs 127 and 128 , and integrate these data with those collected on the JOIDES Resolution by XRF (Tamaki, Pisciotto, Allan, et al., 1990; Ingle, Suyehiro, von Breymann et al., 1990). Our manuscript will serve two purposes: to characterize the compositional nature of the igneous rocks collected during the two legs, and to highlight the compositional changes in the igneous units resulting from pervasive alteration during emplacement and diagenesis. A discussion of the isotopic composition of these samples and the resultant source constraints is given in Cousens and Allan (this volume), and a more complete discussion of certain mineralogical and compositional effects of alteration and diagenesis is given in Allan (this volume). Readers should refer to the Leg 127 and 128 Initial Reports volumes (Tamaki, Pisciotto, Allan, et al., 1990; Ingle, Suyehiro, von Breymann et al., 1990) for supplementary shipboard whole-rock major and trace-element X-ray fluorescence (XRF) analyses and for basic hand-specimen and petrographic descriptions of the analyzed samples. 


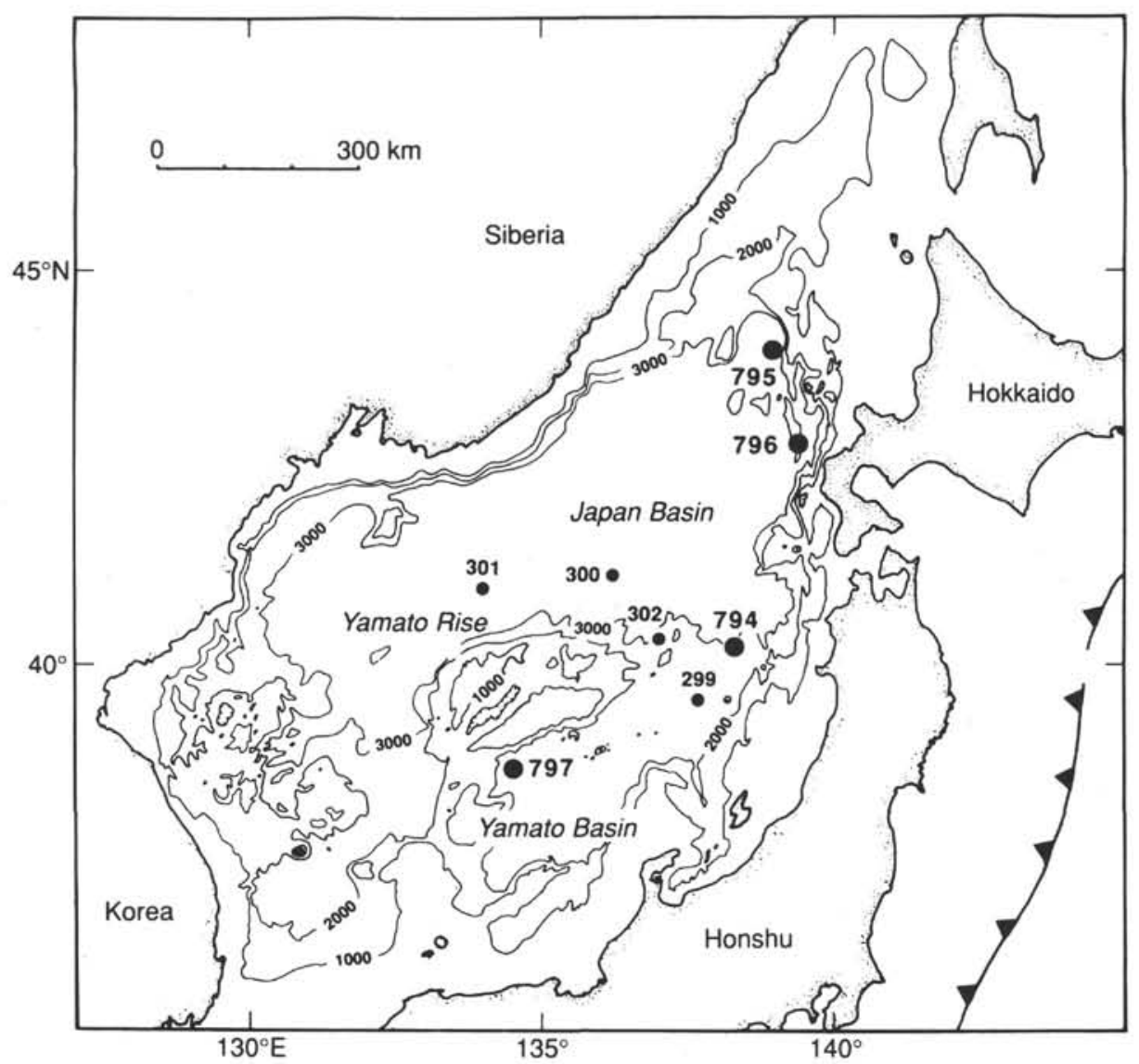

Figure 1. Map showing location of basement Sites 794, 795, and 797; DSDP sites are also shown. Figure is from Tamaki, Pisciotto, Allan, et al. (1990).

\section{DESCRIPTION OF BASEMENT UNITS}

\section{Site 794}

Coring during Legs 127 and 128 recovered nine igneous units, as interpreted by the respective shipboard parties (Figs. 2 and 3). Until further analysis of additional samples on the cored rock can be made, Units 1 to 5 will be considered to have been defined by the Leg 127 shipboard party (Tamaki, Pisciotto, Allan, et al., 1990), and Units 6 to 9 will be considered to have been defined by the Leg 128 shipboard party (Ingle, Suyehiro, von Breymann et al., 1990). In addition, the Leg 128 party defined a unit (Leg 128 Unit 2) not recovered (or recognized) by Leg 127; this unit will be designated here as Unit 2-D.

Units 1 to 6 were interpreted by the Leg 127 shipboard party as sills intruded into soft, tuffaceous, and silty claystones. This interpretation was based on: (1) the presence of baked sediments above and below unit margins, including welding of tuffaceous sediments immediately above Unit 6; (2) the thick nature of the units; (3) the outward fining of unit grain size, from doleritic interiors to finegrained basalts on the unit margins; (4) the lack of rubbly zones at the unit margins; (5) the intense nature of hydrothermal alteration of the units, with the symmetrical alteration at the unit margins that approached or equaled $100 \%$; and (6) the laterally extensive nature of individual horizontal reflectors visible at the top of "acoustic basement" in seismic sections. The shipboard party for Leg 128 came to similar conclusions for some units, but considered others to represent extrusive lavas (Units 1, 2-D, 6, 7, and 8). We comment that if emplacement of the Site 794 sills into shallow, unconsolidated soft sediment followed the model of Einsele (1982) for intrusion of sills in the Guaymas Basin, then the distinction between extrusive lava flows and intrusive sills is likely to be tenuous and difficult to ascertain. ${ }^{40} \mathrm{Ar} /{ }^{39} \mathrm{Ar}$ and ${ }^{40} \mathrm{Ar} /{ }^{36} \mathrm{Ar}$ radiometric determinations of Site 794 units indicate ages of around $20 \mathrm{Ma}$ (Kaneoka et al., this volume; Pouclet and Bellon, this volume). In contrast, the Leg 127 shipboard party estimated the age of the immediately overlying sediments to be 14.8-16.2 Ma based on extrapolation from paleontological control (Tamaki, Pisciotto, Allan, et al., 1990). The thickness of sediment between the units is uncertain, due to poor sediment recovery.

\section{Site 795}

Site 795 recovered three units of moderately to highly altered, brecciated to massive, vesicular, sparsely plagioclase and pyroxene phyric basalts and basaltic andesites (Fig. 4) that the Leg 127 shipboard party (Tamaki, Pisciotto, Allan, et al., 1990) interpreted as representing three eruptions (Units 1 and 2, 3A, and 3B). Unit 2 likely represents the silicified base of Unit $1 .{ }^{40} \mathrm{Ar} /{ }^{39} \mathrm{Ar}$ release patterns from Site 795 samples show disturbed spectra and provide a range of age estimates of 13-24 Ma (Kaneoka et al., this volume). Paleontological data provide estimates of about $14 \mathrm{Ma}$ for the overlying sediments (Tamaki, Pisciotto, Allan, et al., 1990). The eruption depth was probably below $100-200 \mathrm{~m}$, as indicated by the character of the overlying low-energy sedimentary structures. Units 1,2 , and $3 \mathrm{~B}$ underwent autobrecciation upon extrusion, shown by their brecciated character.

\section{Site 797}

Twenty-one igneous units were recovered by coring during Leg 127 (Fig. 5, after Tamaki, Pisciotto, Allan, et al., 1990). Units 1, 2, and 4 were interpreted to be lava flows, as they lack chilled margins 


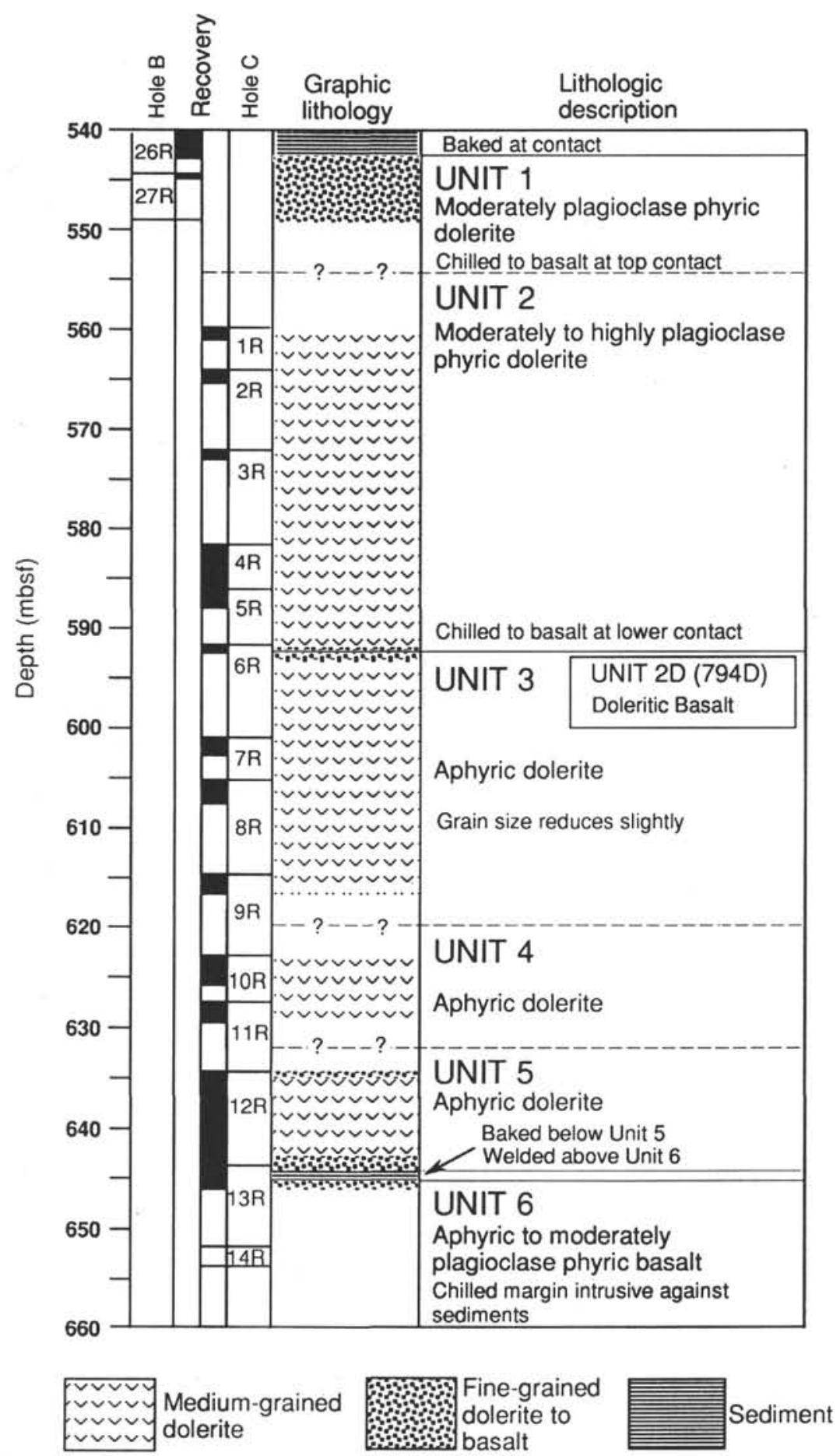

Figure 2. Igneous rock lithology of Site 794 as summarized by Leg 127 shipboard scientific party (Tamaki, Pisciotto, Allan, et al., 1990), with the position of Unit 2D (defined by Ingle, Suyehiro, von Breymann, et al., 1990; see text) shown (mbsf = meters below seafloor).

and are typically highly fractured and brecciated throughout. Unit 1 likely represents the rubbly top of Unit 2 . In contrast, Units 3 and 5 to 21 were interpreted as sills intruded into soft, interlaminated sandstones, siltstones, and silty claystones. This interpretation was based upon the following: (1) the presence of baked sediments above and below unit margins (including the reversal of sediment magnetic orientation); (2) the massive nature of the units; (3) the transition from doleritic or coarse basaltic interiors to fine-grained basalts at the unit margins; (4) the lack of rubbly zones at the unit margins; and (5) the laterally extensive nature of individual horizontal reflectors visible at the top of acoustic basement. Many of these units were intensely altered as well, with the amount of alteration increasing at the unit margins. Extrusion and intrusion likely took place around $18-20 \mathrm{Ma}$, from ${ }^{40} \mathrm{Ar} /{ }^{39} \mathrm{Ar}$ dating of sills (Kaneoka et al., this volume) and the estimated age of the immediately overlying sediments (Tamaki, Pisciotto, Allan, et al., 1990). 


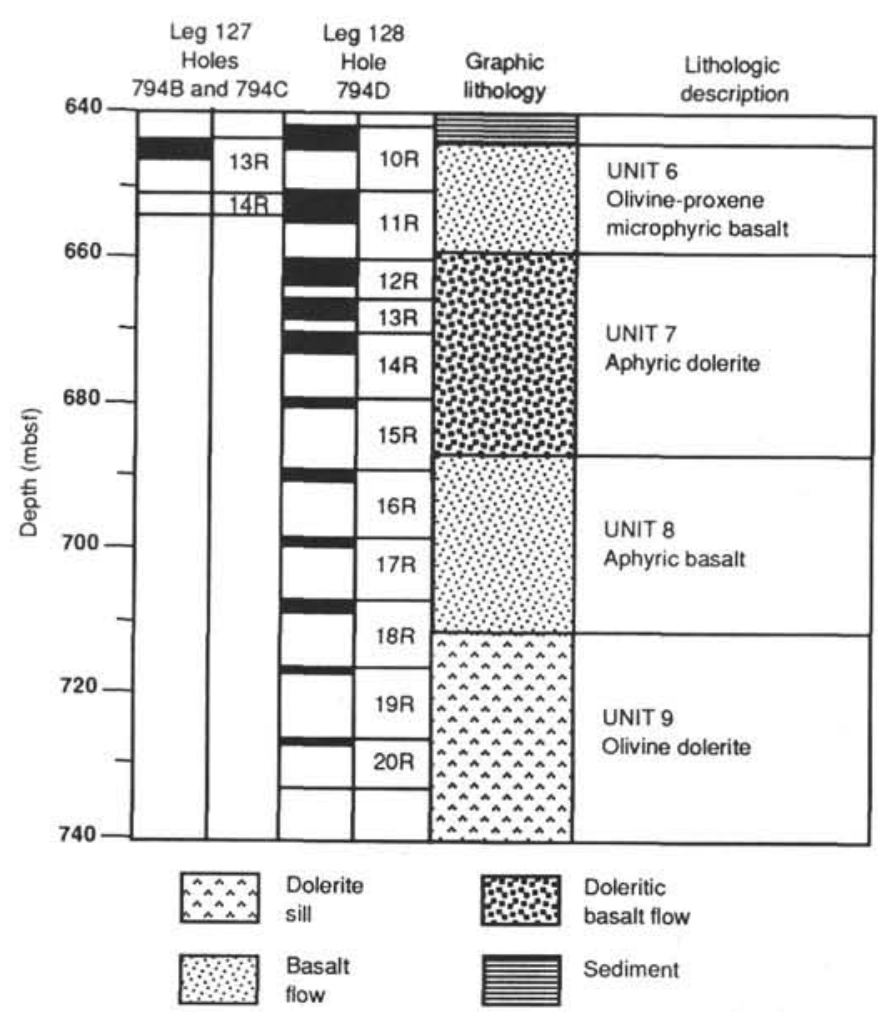

Figure 3. Lower igneous rock lithology of Site 794 as summarized by Leg 128 shipboard party (Ingle, Suyehiro, von Breymann, et al., 1990). Note that igneous units in the tables and figures follow the unit designation scheme of Tamaki, Pisciotto, Allan, et al. (1990) for Units 1 to 5 and Ingle, Suyehiro, von Breymann, et al. (1990) for Units 6 to 9, with the exception that Unit 2 as defined in Hole 794D is referred to as "Unit 2D."

Acoustic basement was determined by Leg 127 to consist of sill/sediment complexes as at Site 794. A better recovery of intervening sediment at Site 797 allowed a more accurate determination of unit boundaries. Drilling extended below the visible seismic reflectors at the site, indicating that the thickness of sill/sediment complexes in the Yamato Basin may be considerably greater than estimated from seismic profiles.

\section{ANALYTICAL METHODS}

All analyzes were of powder split derived from a $10-$ to $20-\mathrm{cm}^{3}$ (pre-crushing volume) whole-rock sample. Samples were sanded with garnet paper to remove saw marks, broken in plastic bags with a hammer to pea-sized material, and then pulverized for 2-5 minutes in a Spex alumina ceramic shatterbox. Acetone was used in grinding when sample caking was a problem. Samples were not ashed after crushing, so comparison to shipboard XRF analyses should take into account changes in weight resulting from shipboard loss-on-ignition procedures.

Powdered samples between 190 and $250 \mathrm{mg}$ each were weighed with a Cahn 4200 electronic balance and sealed into $2 \times 2-\mathrm{cm}$ polyethylene sachets (bags). The sachets were irradiated in the lowflux reactor SLOWPOKE II at the University of Toronto, and counted at 7 and 40 days after irradiation at facilities in the Department of Geology, University of Toronto. The irradiation and analysis procedures generally follow those of Barnes and Gorton (1984) and Stix and Gorton (1990). The samples were calibrated against international rock standards and against pure elements where necessary. The performance of the system was monitored by the in-house basalt standard UTB1 (Barnes and Gorton, 1984). Samples were counted from 3 to 9 hours, with samples containing lower amounts of the rare earth elements (REE) counted for longer periods of time. Aptec coaxial intrinsic germanium detectors of $25 \%$ efficiency were used to count the samples; these have a resolution of $0.8 \mathrm{kev}$ at $122 \mathrm{kev}$. To compensate for the low neutron flux of the reactor and resultant low activity of the samples, a sample/detector distance of $1 \mathrm{~cm}$ was utilized. Geometric problems were minimized in this configuration by using flat sample sachets of less than $1 \mathrm{~mm}$ thickness; relative errors caused by sample geometry are judged to be always below $5 \%$.

After initial analysis of the data had been completed, one of us (JFA) realized that the Cahn balance used for weighing had become uncalibrated because of an electrical power failure prior to sample weighing, resulting in weighing errors of approximately $11 \%$. By weighing with standard weights, this error was determined to be linear in nature. As a result, 26 samples were re-weighed at the University of Toronto and rerun, with results given in Table 1. This second data set contains multiple samples from all earlier dates of weighing and determined that the weighing error was constant. After correction for background and peak overlap interferences, the intensity of gamma ray emission in irradiated samples is directly related to the relative amount of the element of interest (Laul, 1978). Therefore, simple corrections to overcome the weighing error can be made by directly comparing the two data sets. Table 1 also gives the corrected results from the earlier data set, with these samples marked with an asterisk.

Other relative sources of error may exist for both shipboard XRF and shore-based INAA measurements. Compositional variation within a unit may result from crushed samples not being of sufficient volume to fully represent the sampled rock, particularly where the sample is coarse grained. This was not judged to be a problem in the shore-based study, but shipboard analyses were based on crushed material of as little as 6 $\mathrm{cm}^{3}$ in volume. In addition, it should be emphasized that the shore-based studies did not sample the exact interval analyzed by the shipboard studies, although care was taken to obtain samples located adjacent to the sites of shipboard sampling. In the more intensely altered rock, sample heterogeneity could therefore introduce uncertainty.

In addition to the INAA analyses, eight samples from Site 797 were analyzed by X-ray fluorescence by M. Rhodes at the University of Massachusetts and Steve Nelson at Tulane University (Table 2). These samples represent units that were not analyzed on-board ship because of end-of-Leg time constraints, and represent splits of powders analyzed by INAA. A discussion of the analytical procedures and estimated errors is given in Rhodes (1988). Sample powders were ashed before analysis, with loss on ignition (LOI) determined by J. Allan at the Ocean Drilling Program, Texas A\&M University by ashing the rock powder at $1000^{\circ} \mathrm{C}$ for $1 \mathrm{hr}$. Adiscussion of the shipboard XRF procedures is given in Tamaki, Pisciotto, Allan, et al. (1990).

\section{RELATIVE MOBILITY OF ELEMENTS DURING ALTERATION}

\section{Unit 5, Site 794}

All units from Site 794 are variably altered, with the LOI ranging from $1.7 \%$ to $5.6 \%$. Unit 5, a 9.8-m-thick sill, was cored with full recovery in Core $127-794 \mathrm{C}-12 \mathrm{R}$, offering a unique opportunity to see the effects of alteration on rock chemistry. Most alteration likely occurred during and immediately after intrusion of the unit into soft, tuffaceous sediments during unit cooling, but also occurred during diagenesis after unit emplacement (Tamaki, Pisciotto, Allan, et al., 1990; Allan, this volume). The unit varies from a highly altered, coarse, highly vesicular, yet aphyric dolerite in the interior to a nearly totally altered, felty spherulitic, olivine plagioclase phyric basalt at the margin (Fig. 6). The unit interior is dominated by subhedral laths of plagioclase intergrown with ophitic and subophitic clinopyroxene, with most of the clinopyroxene and all of the interstitial mesostasis replaced by secondary brown mixed-layer clays. Secondary irregular blebs of pyrite are common, and secondary adularia as well as brown clays have replaced plagioclase in the unit margins. Figures 7 and 8 show the compositional variation with LOI observed in the shipboard 


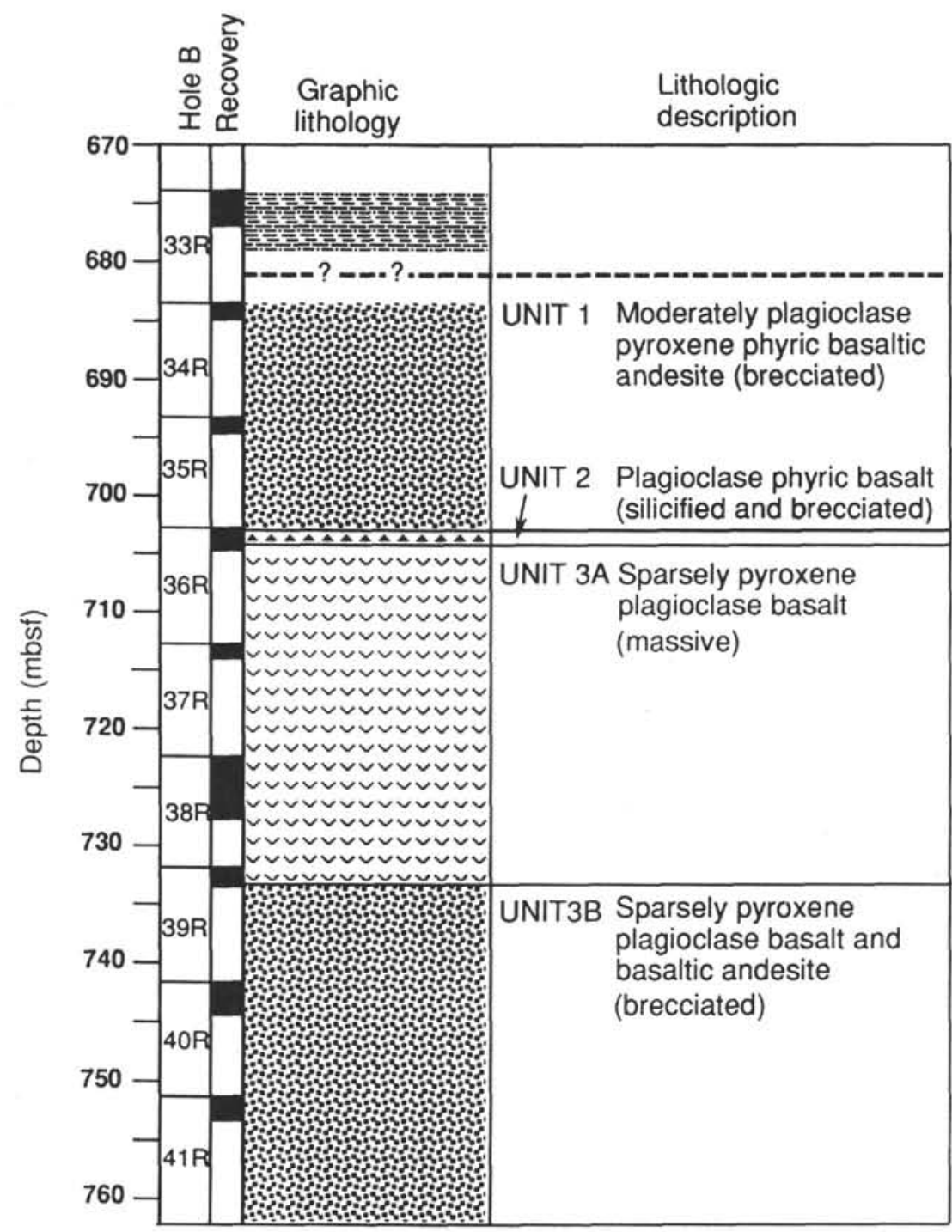

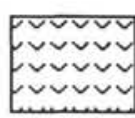

Silicified lava

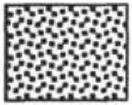

Brecciated lava

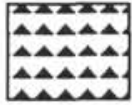

Massive lava

Figure 4. Igneous rock lithology of Site 795 as summarized by Leg 127 shipboard scientific party (Tamaki, Pisciotto, Allan, et al., 1990). Unit 2 likely represents the silicified base of Unit 1.

XRF analyses, while Figure 9 shows the variation in REE and other selected elements from our INAA analyses. Shipboard petrographic observations show that the LOI is a useful proxy for the degree of alteration within a given unit, with samples having higher LOI also having a higher percentage of secondary minerals (Tamaki, Pisciotto, Allan, et al., 1990). In this context, the LOI represents the dehydration of secondary clays and zeolites and the volatilization of secondary carbonate minerals. Large changes in relative abundance of elements with LOI therefore should reflect mobility of elements during alteration, although smaller changes in absolute abundance may be caused by crystal settling or floatation during emplacement of the units.

Table 1 and Figure 7 show that $\mathrm{K}_{2} \mathrm{O}, \mathrm{MgO}$, and $\mathrm{CaO}$ are strikingly affected by alteration, resulting in profound within-unit variation. The coupled increase in $\mathrm{MgO}$ and the decrease in $\mathrm{CaO}$ can be related to the formation of mixed-layer secondary clays from the alteration of either glassy mesostasis or olivine and pyroxene, with $\mathrm{CaO}$ being excluded from the clay mineral structure (Allan, this volume; Seyfried, 1987). This alteration results in the rock $\mathrm{Mg}$ number $\left(\mathrm{Mg}^{2+} /\left(\mathrm{Mg}+\mathrm{Fe}^{2+}\right)\right.$ ratio strongly increasing with LOI (Fig. 7), despite the fact that Fe increases slightly with alteration as well. $\mathrm{Sr}$, geochemically similar to $\mathrm{Ca}$, also decreases sharply with LOI, as does Co (Figs. 8 and 9). The increase in $\mathrm{K}_{2} \mathrm{O}$ is also likely associated with the formation of these clays; several shipboard XRD analyses of clay separates suggest the presence of both mixed-layer and kaolinitic clays (Tamaki, Pisciotto, Allan, et al., 1990). The large-ion lithophile element (LILE) Rb shows a particularly strong increase with increasing LOI (Fig. 8), a relationship that has been previously noted for low-temperature alteration of basalt by seawater (Hart et al., 1974). Y and Sc show much larger variability than expected, as they are not considered to be easily mobilized by fluids (e.g. Pearce, 1983; Tatsumi et al., 1986). In particular, Y shows several sharp changes 


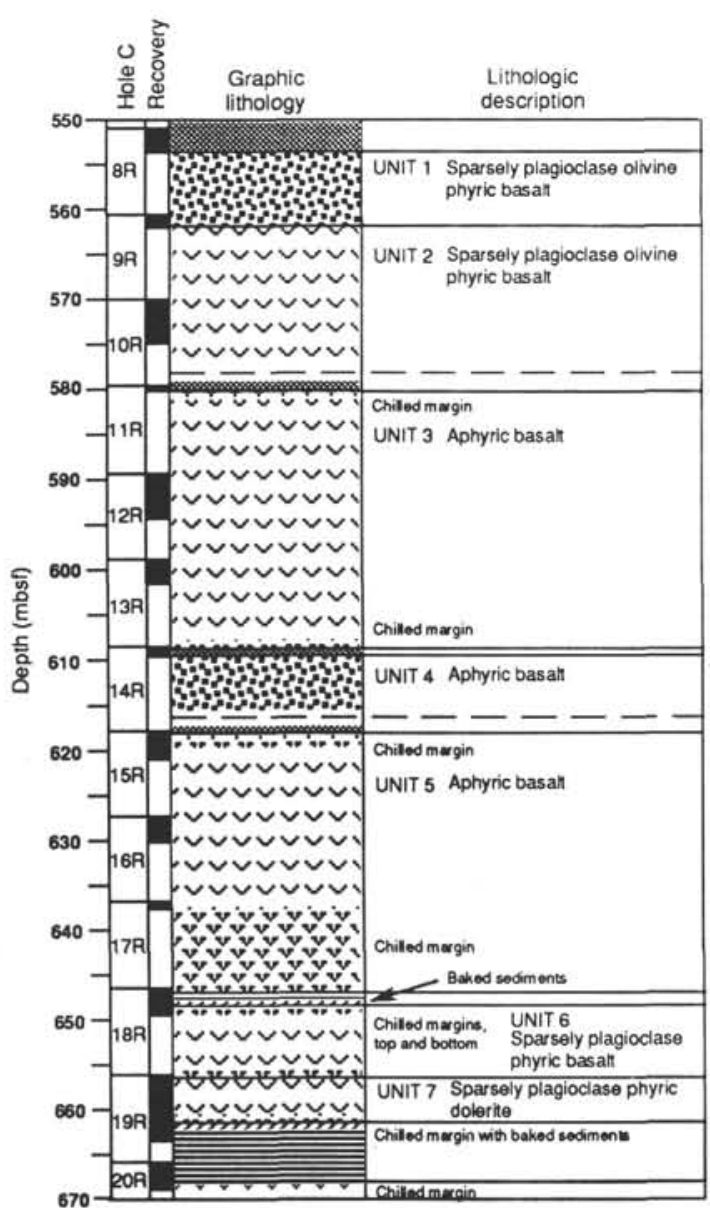

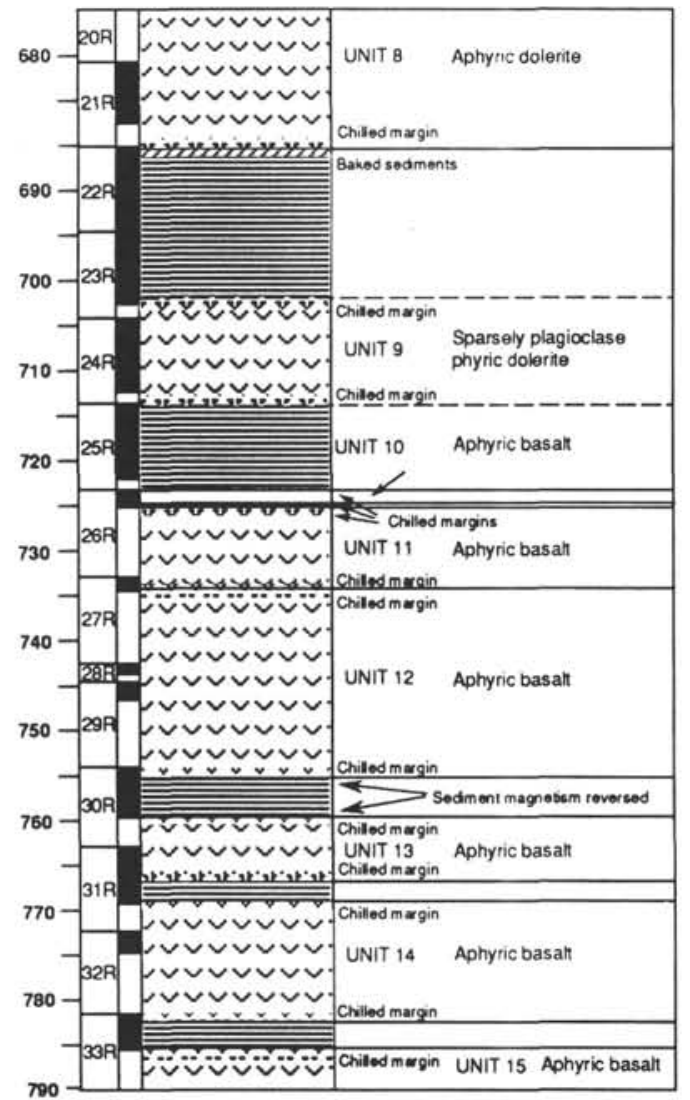

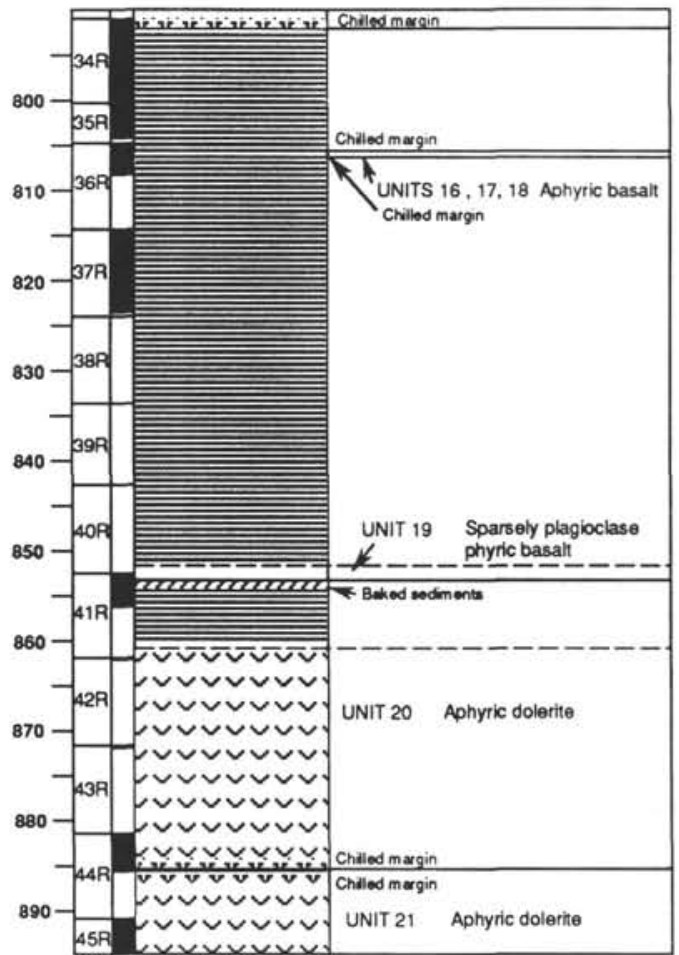

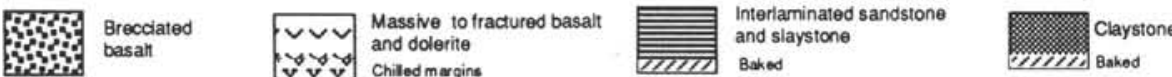

Figure 5. Igneous rock lithology of Site 797 as summarized by Leg 127 shipboard scientific party (Tamaki, Pisciotto, Allan, et al., 1990). 
in content within a few units, shown effectively by changes in $\mathrm{Zr} / \mathrm{Y}$ (Tables 1 and 2; Fig. 10). However, Bienvenu et al. (1990) also found evidence for $\mathrm{Y}$ mobility during low-temperature seawater alteration of glassy MORB. A key observation is that the elemental variations due to alteration are expressed reciprocally in the respective concentrations in sediment pore waters above the intrusive units, with strong gradients of enrichments in pore water $\mathrm{Sr}$ and $\mathrm{Ca}$ and depletions in $\mathrm{Mg}$ and $\mathrm{K}$ above the igneous units (Tamaki, Pisciotto, Allan, et al., 1990). These data indicate that the dolerites and basalts serve as both a sink and a source for elemental exchange with aqueous fluids in the overlying sediments.

Other elements show little or no variation with LOI. These elements include most high-field strength elements (HFSE; $\mathrm{Zr}, \mathrm{Nb}, \mathrm{Hf}$, and $\mathrm{Ta}$ ), $\mathrm{Th}, \mathrm{Ni}$, and $\mathrm{Cr}$ (Figs. 8 and 9). As a result of their relatively immobile nature, Bienvenu et al. (1990) have recently proposed that the highly incompatible/moderately incompatible element ratios $\mathrm{Nb} / \mathrm{Zr}$ and $\mathrm{Ta} / \mathrm{Hf}$ are potentially more useful in altered basalts than the REE ratio $\mathrm{La} / \mathrm{Sm}$, and indeed ratios of these elements appear constant in Unit 5 (Fig. 10; Table 1). Relative absolute abundance variations of up to $20 \%$ exist for the REE within Unit 5 of Site 794 (Fig. 9), but the light-to-medium rare-earth elements (LREE to MREE) in a given sample covary in abundance (i.e., the chondrite-normalized REE patterns given in Figure 15 are generally parallel from $\mathrm{La}$ to $\mathrm{Tb}$ ). Slight depletions in the heavy rare-earth elements (HREE) are noted for the most altered sample (Section 127-794C-13R-1, $19 \mathrm{~cm}$; Fig. 9). There is no relation of this relative abundance variation to either LOI or stratigraphic position.

One approach to determining the relative mobility of a given element is to normalize its concentration against an element that is "conserved" during alteration, a method used previously to study problems of rock alteration (Hynes, 1980; Fowler, 1990) and analogous to the use of Pearce element ratios in modeling magmatic liquid lines of descent (Pearce, 1968; Russell and Nicholls, 1988; Nicholls and Russell, 1990). This approach should circumvent problems of volume change caused by infilling of vesicles with alteration products, and the volume change associated with alteration of the sample to lower-density, hydrous minerals. Hf is analyzed with high precision by INAA (Laul, 1978), and has proved to be highly immobile during alteration processes by numerous studies (e.g., Bienvenu et al., 1990; Humphris and Thompson, 1978; Wood et al., 1979). Figure 10 shows normalization of the REE and Th to Hf, to see if any systematic changes with LOI are present. Th appears to have been lowered slightly by alteration processes, although the magnitude is small $(\approx 15 \%)$. Small variations exist between the REE, with all normalized REE abundances except Ce slightly declining with alteration and the HREE showing the greatest decline. The chondrite-normalized ratio $\mathrm{La} / \mathrm{Yb}$ (Fig. 10) records the slight relative HREE depletion as compared to the LREE. However, the chondrite-normalized ratio $\mathrm{La} / \mathrm{Sm}$ remains fairly constant.

Overall, the REE do not appear to have been highly mobile during these conditions of alteration, determined by Allan (this volume) to be subgreenschist in nature at conditions of less than $230^{\circ} \mathrm{C}$ in temperature, with alteration temperatures in Units 6 to 8 of Hole 794D determined from mineralogical and oxygen isotope data to be $65^{\circ}-$ $180^{\circ} \mathrm{C}$ (Proust et al, this volume). This finding of non-mobile REE behavior agrees well with the higher temperature $\left(500^{\circ}-600^{\circ} \mathrm{C}\right)$ experimental results of Hajash (1984), but contrasts strongly with the findings and suggestions of Frey et al. (1974), Ludden and Thompson (1979), and Bienvenu et al. (1990), who report significant additions and depletions in relative LREE and MREE abundance during longterm, low-temperature (ambient seawater) alteration of glassy midocean ridge basalt (MORB) flow rinds. Juteau et al. (1979) studied basalts from DSDP Hole 417A with an intensity of alteration similar to those of Site 794 and found strong LREE enrichments associated with alteration, at strong variance with our results presented here.

\section{Unit 3, Site 797}

Alteration of units from Site 797 vary, with LOI ranging from $1.6 \%$ to $8.4 \%$. Unit 3 , a $28-\mathrm{m}$ - thick sill intruded into laminated claystones at Site 797, allows another opportunity to look at element variation with alteration within a given igneous unit (Tamaki, Pisciotto, Allan, et al., 1990). In contrast to Unit 5 at Site 794, the unit is defined over several core lengths with relatively poor recovery (Fig. 5), making it a less-reliable indicator of elemental variation. The interior of Unit 3 consists of a slightly altered, fine-to-medium grained, aphyric basalt (Fig. 6). Clinopyroxene exhibits subophitic and radiating sheaf and comb textures around and between plagioclase laths and subhedral olivine and magnetite. The interstitial mesostasis is partially to completely replaced by green clays, as are the silicate phases to a much smaller extent. The margins are very highly to totally altered spherulitic to microlitic basalts, with the plagioclase microlites replaced by sodic plagioclase and the plagioclase phenocrysts by adularia. Conditions of alteration are similar to those of Unit 5, Site 794 (Allan, this volume).

Figures 11, 12, and 13 show the elemental variations within Unit 3 of Site $797 . \mathrm{MgO}$ and $\mathrm{CaO}$ again show strong coupled enrichment and depletion with LOI. However, $\mathrm{FeO}$ shows a decline with decreasing LOI, and $\mathrm{K}_{2} \mathrm{O}$ again increases with LOI at the unit margin. Most HFSE show little change, but Co shows a strong enrichment with LOI, completely opposite to that shown by Unit 5 of Site 794 (Fig. 13). Rb again also shows a strong enrichment with LOI (Fig. 12). Y appears to be mobile, with significant variation in $\mathrm{Zr} / \mathrm{Y}$ (Fig. 14).

The effects on the REE as compared to Unit 5 of Site 794 are more pronounced. Ce is enriched and the HREE Yb and Lu (geochemically similar to Y) are strongly depleted in the most-altered sample from the unit, resulting in an unusual chondrite-normalized REE pattern that crosses those of the other Unit 3 samples (Fig. 19). Frey et al. (1974) found similar evidence of HREE mobility during alteration. Evidence for the mobility of Ce during low-temperature alteration of basalt by seawater has been cited by previous authors (Frey et al., 1974; Ludden and Thompson, 1979; Juteau et al., 1979; and Bienvenu et al., 1990), and the normalized Ce/Hf ratio (Fig. 14) as well as the absolute abundance data (Table 1) argues strongly that it has indeed been enriched in the Unit 3 altered margin. The depletion in the HREE $\mathrm{Yb}$ and $\mathrm{Lu}$ is also strikingly shown by their normalized ratios relative to $\mathrm{Hf}$ and by their chondrite-normalized $\mathrm{La} / \mathrm{Yb}$ ratio (Fig. 14).

\section{Discussion}

There are two results from this overview of the effects of alteration and diagenesis on Leg 127/128 rock chemistry. First, it is clear that the effects of rock alteration on the chemistry of the Leg 127 and Leg 128 rocks are profound, and that key petrogenetic indicators such as the large-ion lithophile elements (LILE), $\mathrm{Ca}, \mathrm{Mg}$, and $\mathrm{Sr}$ have been somewhat redistributed in even the fresher samples. In the most altered samples, key elements such as $\mathrm{Y}, \mathrm{Ce}, \mathrm{Yb}$, and $\mathrm{Lu}$ were also mobile. Although not specifically discussed, Units 7 and 8 from Hole 797C (Table 2) also allow for a comparison between totally altered and moderate- to highly-altered samples from the same unit. These two examples show that the LREE may increase during conditions of extreme alteration. As Allan (this volume) demonstrated for several specific Leg 127 igneous units, investigators must be cautious before interpreting that any sample from these units closely approximates the composition of a silicate liquid. As a result, it is problematical to construct useful liquid-line-of-descent calculations using the contents of most major elements and many trace elements of units from Legs 127 and 128. Second, excepting the most altered unit margins, the HFSE and the REE do not appear to have been highly mobile under these conditions of subgreenschist alteration and diagenesis. This 
Table 1. Whole-rock geochemical data.

\begin{tabular}{|c|c|c|c|c|c|c|c|c|c|c|c|c|c|}
\hline Hole & $794 B$ & $794 C$ & $794 \mathrm{C}$ & $794 \mathrm{C}$ & 794D & 794D & $794 \mathrm{C}$ & $794 \mathrm{C}$ & $794 \mathrm{C}$ & 794D & 794D & $794 \mathrm{C}$ & $794 \mathrm{C}$ \\
\hline Section & $26 \mathrm{R}-2$ & IR-I & 2R-1 & $4 \mathrm{R}-\mathrm{I}$ & $3 R-3$ & $4 \mathrm{R}-1$ & $7 \mathrm{R}-1$ & $8 \mathrm{R}-1$ & 9R-I & 7R-2 & 9R-2 & $12 \mathrm{R}-1$ & $12 \mathrm{R}-3$ \\
\hline XRF Interval & $101-103$ & $48-50$ & $34-36$ & $42-44$ & $89-94$ & $32-37$ & $46-48$ & $65-67$ & $130-132$ & $8-10$ & $16-20$ & $25-27$ & $71-73$ \\
\hline INNA Interval & $101-103$ & $47-49 *$ & $35-38$ & $47-51^{*}$ & $84-86^{*}$ & $44-46^{*}$ & $24-27$ & $57-61^{*}$ & $131-136$ & $2-4^{*}$ & $9-11^{*}$ & $12-15$ & $68-73$ \\
\hline Unit & 1 & 2 & 2 & 2 & 2 & $2 \mathrm{D}$ & 3 & 3 & 3 & 4 & 4 & 5 & 5 \\
\hline \multicolumn{14}{|l|}{ XRF } \\
\hline $\mathrm{SiO}_{2}$ & 51.67 & 50.82 & 50.71 & 51.21 & 50.04 & 52.10 & 51.53 & 51.65 & 51.46 & 51.27 & 51.23 & 51.35 & 50.06 \\
\hline $\mathrm{TiO}_{2}$ & 1.49 & 1.37 & 1.37 & 1.09 & 1.27 & 1.06 & 1.26 & 1.13 & 1.18 & 1.44 & 1.40 & 1.26 & 1.19 \\
\hline $\mathrm{Al}_{2} \mathrm{O}_{3}$ & 18.23 & 18.45 & 18.77 & 20.23 & 18.11 & 17.36 & 17.80 & 17.70 & 17.86 & 16.56 & 17.23 & 17.08 & 17.69 \\
\hline $\mathrm{Fe}_{2} \mathrm{O}_{3}$ & 9.62 & 10.23 & 9.00 & 8.68 & 10.01 & 8.56 & 8.70 & 8.20 & 8.98 & 9.13 & 9.11 & 9.09 & 9.51 \\
\hline $\mathrm{MnO}$ & 0.09 & 0.17 & 0.13 & 0.13 & 0.12 & 0.23 & 0.15 & 0.14 & 0.16 & 0.12 & 0.13 & 0.10 & 0.13 \\
\hline $\mathrm{MgO}$ & 8.48 & 7.02 & 5.68 & 5.98 & 6.57 & 11.04 & 9.81 & 9.81 & 11.46 & 10.63 & 11.28 & 12.00 & 10.19 \\
\hline $\mathrm{CaO}$ & 3.47 & 6.83 & 9.33 & 10.60 & 9.50 & 5.41 & 6.21 & 7.18 & 5.80 & 8.18 & 6.77 & 5.28 & 9.06 \\
\hline $\mathrm{Na}_{2} \mathrm{O}$ & 4.05 & 3.44 & 3.78 & 3.39 & 3.25 & 3.24 & 3,89 & 3.35 & 3.48 & 2.98 & 3.25 & 3.27 & 2.67 \\
\hline $\mathrm{K}_{2} \mathrm{O}$ & 2.68 & 1.50 & 0.68 & 0.60 & 0.62 & 1.54 & 0.82 & 0.73 & 0.67 & 0.59 & 0.64 & 1.40 & 0.28 \\
\hline $\mathrm{P}_{2} \mathrm{O}_{5}$ & 0.30 & 0.26 & 0.30 & 0.22 & 0.21 & 0.17 & 0.20 & 0.20 & 0.19 & 0.21 & 0.20 & 0.23 & 0.21 \\
\hline Total & 100.08 & 100.09 & 99.75 & 102.13 & 99.70 & 100.71 & 100.37 & 100.09 & 101.24 & 101.11 & 101.24 & 101.06 & 100.99 \\
\hline LOI & 4.22 & 2.23 & 1.72 & 1.86 & 1.73 & 4.70 & 2.52 & 3.17 & 3.68 & 2.60 & 3.02 & 3.53 & 2.52 \\
\hline $\mathrm{Mg} \#$ & 0.66 & 0.60 & 0.58 & 0.60 & 0.54 & 0.69 & 0.71 & 0.73 & 0.73 & 0.67 & 0.69 & 0.74 & 0.70 \\
\hline $\mathrm{Cr}$ & 33 & 40 & 51 & 63 & 79 & 358 & 229 & 259 & 364 & 216 & 266 & 236 & 235 \\
\hline $\mathrm{Ni}$ & 15 & 11 & 15 & 17 & 21 & 182 & 100 & 132 & 165 & 88 & 126 & 69 & 91 \\
\hline V & 270 & 263 & 245 & 212 & 238 & 257 & 309 & 259 & 297 & 289 & 278 & 262 & 245 \\
\hline $\mathrm{Cu}$ & 22 & 77 & 44 & 40 & 34 & 39 & 62 & 53 & 56 & 56 & 51 & 95 & 51 \\
\hline $\mathrm{Zn}$ & 45 & 286 & 75 & 66 & 49 & 57 & 69 & 67 & 71 & 36 & 49 & 52 & 59 \\
\hline $\mathrm{Rb}$ & 11.3 & 21 & 7.1 & 7 & 7 & 9 & 10.3 & 11 & 7.3 & 4 & 5 & 8.8 & 1.2 \\
\hline $\mathrm{Sr}$ & 257 & 349 & 387 & 405 & 370 & 238 & 273 & 260 & 255 & 133 & 277 & 238 & 278 \\
\hline $\mathrm{Y}$ & 18.6 & 20.4 & 21.2 & 17.6 & 20.0 & 21.0 & 17.9 & 17.5 & 17.0 & 22.0 & 20.0 & 21.7 & 19.8 \\
\hline $\mathrm{Zr}$ & 103 & 97 & 103 & 88 & 90 & 85 & 92 & 90 & 83 & 100 & 99 & 101 & 92 \\
\hline $\mathrm{Nb}$ & 8 & 8 & 8 & 7 & 7 & 4 & 5 & 5 & 4 & 11 & 9 & 8 & 8 \\
\hline $\mathrm{Ba}$ & 166 & 274 & 162 & 135 & 154 & 143 & 166 & 160 & 154 & 133 & 121 & 142 & 98 \\
\hline $\mathrm{Ce}$ & 23 & 21 & 27 & 28 & 14 & 19 & 28 & 26 & 16 & 35 & 22 & 21 & 18 \\
\hline \multicolumn{14}{|l|}{ INNA } \\
\hline $\mathrm{Na}_{2} \mathrm{O}$ & 3.37 & 2.77 & 3.35 & 2.96 & 3.00 & 2.66 & 3.13 & 3.00 & 3.11 & 2.69 & 2.76 & 3.04 & 2.55 \\
\hline $\mathrm{Fe}_{2} \mathrm{O}_{3} 7$ day & 9.17 & 9.77 & 8.13 & 7.55 & 9.09 & 7.79 & 8.63 & 7.84 & 8.69 & 7.60 & 7.99 & 8.36 & 7.84 \\
\hline $\mathrm{Fe}_{2} \mathrm{O}_{3} 40$ day & 9.12 & 9.74 & 8.30 & 7.56 & 9.02 & 7.73 & 8.60 & 7.85 & 8.62 & 7.61 & 7.97 & 8.37 & 7.57 \\
\hline $\mathrm{Sc}$ & 29.1 & 29.8 & 29.6 & 27.6 & 32.8 & 28.4 & 30.7 & 29.7 & 33.2 & 34.6 & 31.3 & 34.9 & 33.8 \\
\hline $\mathrm{Cr}$ & 58 & 56 & 69 & 101 & 119 & 308 & 276 & 318 & 358 & 302 & 284 & 249 & 263 \\
\hline Co & 25.0 & 29.6 & 28.1 & 23.1 & 30.2 & 43.1 & 26.1 & 37.2 & 25.2 & 29.8 & 33.3 & 35.8 & 35.4 \\
\hline $\mathrm{Ni}$ & 5 & & & & & & 92 & & 132 & & & 54 & 92 \\
\hline $\mathrm{Zn}$ & 49 & 288 & 74 & 60 & 61 & 70 & 61 & 64 & 80 & 62 & 68 & 60 & 50 \\
\hline $\mathrm{Rb}$ & 9.6 & 21 & 6.3 & 4 & 7 & 2 & 7.1 & 14 & 10.4 & 7 & 7 & 11.3 & 1.8 \\
\hline $\mathrm{Sr}$ & 267 & 305 & 377 & 357 & 377 & 195 & 233 & 200 & 228 & 250 & 195 & 235 & 216 \\
\hline $\mathrm{Ba}$ & 197 & 221 & 139 & 123 & 118 & 92 & 115 & 141 & 131 & 105 & 116 & 111 & 70 \\
\hline $\mathrm{La}$ & 8.99 & 8.51 & 9.15 & 7.37 & 7.99 & 6.39 & 6.46 & 6.46 & 7.42 & 8.89 & 7.93 & 8.95 & 7.12 \\
\hline $\mathrm{Ce}$ & 21.4 & 21.0 & 22.6 & 17.6 & 18.8 & 16.5 & 14.8 & 13.8 & 17.3 & 21.3 & 18.5 & 21.1 & 17.8 \\
\hline Nd & 12.0 & 12.3 & 11.1 & 10.0 & 11.9 & 9.5 & 8.1 & 8.3 & 10.4 & 11.7 & 9.0 & 11.2 & 8.5 \\
\hline $\mathrm{Sm}$ & 3.31 & 3.34 & 3.33 & 2.90 & 3.15 & 2.80 & 2.39 & 2.54 & 2.89 & 3.18 & 2.76 & 3.31 & 2.75 \\
\hline $\mathrm{Eu}$ & 1.25 & 1.20 & 1.30 & 1.14 & 1.25 & 1.03 & 0.91 & 0.98 & 1.08 & 1.12 & 0.98 & 1.17 & 0.95 \\
\hline $\mathrm{Tb}$ & 0.545 & 0.632 & 0.575 & 0.483 & 0.551 & 0.578 & 0.420 & 0.478 & 0.534 & 0.562 & 0.465 & 0.602 & 0.454 \\
\hline $\mathrm{Yb}$ & 1.53 & 2.04 & 2.07 & 1.55 & 1.81 & 1.82 & 2.14 & 1.87 & 2.06 & 1.91 & 1.03 & 2.28 & 1.66 \\
\hline Lu & 0.253 & 0.312 & 0.315 & 0.261 & 0.310 & 0.272 & 0.298 & 0.274 & 0.315 & 0.305 & 0.273 & 0.330 & 0.275 \\
\hline $\mathrm{Hf}$ & 2.08 & 2.12 & 2.18 & 1.70 & 1.92 & 1.84 & 1.87 & 1.80 & 2.09 & 2.31 & 1.86 & 2.23 & 1.91 \\
\hline $\mathrm{Ta}$ & 0.501 & 0.548 & 0.561 & 0.465 & 0.492 & 0.287 & 0.227 & 0.244 & 0.265 & 0.561 & 0.533 & 0.471 & 0.413 \\
\hline $\mathrm{Au}(\mathrm{ppb})$ & 7.17 & 1.76 & 9.45 & 1.32 & 0.60 & 1.62 & 8.89 & 1.14 & 8.32 & 0.83 & 1.91 & 8.97 & 8.23 \\
\hline Th & 0.87 & 0.89 & 0.82 & 0.66 & 0.64 & 0.95 & 0.91 & 0.90 & 0.95 & 1.03 & 0.97 & 0.98 & 0.90 \\
\hline $\mathrm{U}$ & 0.41 & 0.34 & 0.20 & 0.18 & 0.18 & 0.27 & 0.20 & 0.16 & 0.10 & 0.24 & 0.22 & 0.62 & 0.24 \\
\hline $\mathrm{Fe} / \mathrm{Mn}$ & 97 & 54 & 63 & 60 & 75 & 34 & 52 & 53 & 51 & 69 & 63 & 82 & 66 \\
\hline $\mathrm{Ti} / \mathrm{Zr}$ & 86.7 & 84.7 & 79.7 & 74.3 & 84.6 & 74.8 & 82.1 & 75.3 & 85.2 & 86.3 & 84.8 & 74.8 & 77.5 \\
\hline $\mathrm{Zr} / \mathrm{Y}$ & 5.54 & 4.75 & 4.86 & 5.00 & 4.50 & 4.05 & 5.14 & 5.14 & 4.88 & 4.55 & 4.95 & 4.65 & 4.65 \\
\hline$(\mathrm{Nb} / \mathrm{Zr})_{\mathrm{N}}$ & 1.16 & 1.11 & 1.15 & 1.08 & 1.11 & 0.67 & 0.70 & 0.79 & 0.67 & 1.56 & 1.29 & 1.11 & 1.17 \\
\hline$(\mathrm{Ta} / \mathrm{Hf})_{\mathrm{N}}$ & 1.88 & 2.02 & 2.00 & 2.13 & 2.00 & 1.21 & 0.95 & 1.06 & 0.99 & 1.89 & 2.23 & 1.64 & 1.68 \\
\hline$(\mathrm{La} / \mathrm{Sm})_{\mathrm{N}}$ & 1.66 & 1.55 & 1.67 & 1.55 & 1.55 & 1.39 & 1.65 & 1.55 & 1.56 & 1.70 & 1.75 & 1.65 & 1.58 \\
\hline$(\mathrm{La} / \mathrm{Yb})_{\mathrm{N}}$ & 3.87 & 2.75 & 2.91 & 3.13 & 2.91 & 2.31 & 1.99 & 2.28 & 2.37 & 3.07 & 5.05 & 2.59 & 2.83 \\
\hline $\mathrm{Ce} / \mathrm{Ta}$ & 43 & 38 & 40 & 38 & 38 & 57 & 65 & 57 & 65 & 38 & 35 & 45 & 43 \\
\hline $\mathrm{Eu} / \mathrm{Eu}^{*}$ & 1.05 & 1.02 & 1.06 & 1.07 & 1.06 & 1.02 & 1.05 & 1.05 & 1.04 & 1.02 & 1.03 & 1.02 & 1.02 \\
\hline
\end{tabular}


Table 1 (continued).

\begin{tabular}{|c|c|c|c|c|c|c|c|c|c|c|c|c|c|}
\hline Hole & $794 \mathrm{C}$ & $794 \mathrm{C}$ & $794 \mathrm{C}$ & $794 \mathrm{C}$ & $794 \mathrm{D}$ & $794 \mathrm{D}$ & $794 \mathrm{D}$ & $794 \mathrm{D}$ & $794 \mathrm{D}$ & $794 \mathrm{D}$ & $795 B$ & $795 \mathrm{~B}$ & $795 \mathrm{~B}$ \\
\hline Section & $12 R-4$ & $12 \mathrm{R}-7$ & $13 \mathrm{R}-1$ & 13R-2 & $11 \mathrm{R}-1$ & 13R-1 & $16 \mathrm{R}-1$ & $18 \mathrm{R}-1$ & $19 \mathrm{R}-1$ & $20 \mathrm{R}-1$ & $34 \mathrm{R}-1$ & $38 \mathrm{R}-1$ & $39 \mathrm{R}-1$ \\
\hline XRF Interval & $85-86$ & $17-19$ & $21-23$ & 93-95 & $89-95$ & $81-84$ & $67-70$ & $44-47$ & $11-14$ & $20-23$ & $121-123$ & $23-25$ & $9-11$ \\
\hline INNA Interval & $84-89$ & $19-24$ & $19-24$ & $92-96^{*}$ & $86-88$ & $83-85$ & $59-62 *$ & $39-41^{*}$ & $2-4^{*}$ & $24-26 *$ & $121-125^{*}$ & $24-28$ & $9-11$ \\
\hline Unit & 5 & 5 & 5 & 6 & 6 & 7 & 8 & 8 & 9 & 9 & 1 & $3 \mathrm{~A}$ & $3 A$ \\
\hline \multicolumn{14}{|l|}{ XRF } \\
\hline $\mathrm{SiO}_{2}$ & 50.96 & 50.47 & 50.69 & 48.78 & 48.58 & 48.64 & 50.01 & 49.37 & 48.42 & 47.73 & 54.50 & 50.51 & 51.46 \\
\hline $\mathrm{TiO}_{2}$ & 1.32 & 1.16 & 1.14 & 1.21 & 1.11 & 1.51 & 1.63 & 1.67 & 1.51 & 1.48 & 0.99 & 1.00 & 0.99 \\
\hline $\mathrm{Al}_{2} \mathrm{O}_{3}$ & 16.97 & 16.39 & 16.32 & 16.86 & 16.15 & 15.04 & 20.11 & 17.80 & 17.30 & 16.65 & 21.16 & 19.25 & 19.15 \\
\hline $\mathrm{Fe}_{2} \mathrm{O}_{3}$ & 8.18 & 9.83 & 9.78 & 11.34 & 10.36 & 11.55 & 8.49 & 8.68 & 10.53 & 10.64 & 7.50 & 8.62 & 7.77 \\
\hline $\mathrm{MnO}$ & 0.16 & 0.12 & 0.09 & 0.15 & 0.16 & 0.18 & 0.22 & 0.27 & 0.20 & 0.21 & 0.14 & 0.24 & 0.22 \\
\hline $\mathrm{MgO}$ & 9.42 & 14.00 & 14.69 & 13.25 & 12.43 & 8.26 & 7.65 & 7.39 & 7.99 & 9.36 & 4.08 & 6.01 & 6.02 \\
\hline $\mathrm{CaO}$ & 9.09 & 4.52 & 2.54 & 3.40 & 7.75 & 10.63 & 7.91 & 10.76 & 10.61 & 10.18 & 7.21 & 9.84 & 9.73 \\
\hline $\mathrm{Na}_{2} \mathrm{O}$ & 2.84 & 2.85 & 2.53 & 2.65 & 2.49 & 2.83 & 3.34 & 3.02 & 2.91 & 2.82 & 3.95 & 3.05 & 3.45 \\
\hline $\mathrm{K}_{2} \mathrm{O}$ & 0.71 & 1.52 & 3.30 & 1.72 & 0.25 & 0.24 & 0.45 & 0.22 & 0.20 & 0.22 & 0.36 & 0.49 & 0.30 \\
\hline $\mathrm{P}_{2} \mathrm{O}_{5}$ & 0.23 & 0.19 & 0.19 & 0.20 & 0.15 & 0.16 & 0.25 & 0.19 & 0.17 & 0.17 & 0.33 & 0.26 & 0.27 \\
\hline Total & 99.88 & 101.05 & 101.27 & 99.56 & 99.43 & 99.04 & 100.06 & 99.37 & 99.84 & 99.46 & 100.22 & 99.27 & 99.36 \\
\hline LOI & 2.05 & 4.09 & 5.45 & 5.56 & 4.02 & 0.76 & 4.57 & 2.26 & 2.44 & 2.18 & 4.75 & 2.84 & 3.04 \\
\hline Mg\# & 0.72 & 0.76 & 0.77 & 0.72 & 0.68 & 0.56 & 0.61 & 0.59 & 0.57 & 0.61 & 0.55 & 0.61 & 0.63 \\
\hline $\mathrm{Cr}$ & 214 & 223 & 224 & 316 & 377 & 324 & 329 & 319 & 312 & 291 & 0 & 25 & 25 \\
\hline $\mathrm{Ni}$ & 80 & 81 & 97 & 132 & 147 & 129 & 113 & 124 & 137 & 124 & 5 & 26 & 29 \\
\hline V & 294 & 252 & 233 & 271 & 256 & & 278 & 281 & 232 & 234 & 103 & 324 & 296 \\
\hline $\mathrm{Cu}$ & 60 & 31 & 58 & 60 & 54 & 63 & 55 & 74 & 60 & 56 & 8 & 51 & 58 \\
\hline $\mathrm{Zn}$ & 67 & 74 & 72 & 68 & 47 & 85 & 108 & 83 & 81 & 53 & 98 & 91 & 98 \\
\hline $\mathrm{Rb}$ & 6.0 & 9.9 & 17.6 & 9 & 0.4 & 3.0 & 5 & 3 & 3 & 2 & 5 & 11.3 & 4.1 \\
\hline $\mathrm{Sr}$ & 275 & 204 & 139 & 190 & 277 & 194 & 263 & 200 & 185 & 173 & 336 & 259 & 243 \\
\hline $\mathrm{Y}$ & 23.0 & 16.8 & 13.8 & 12.2 & 16.0 & 31.0 & 29.0 & 33.0 & 29.0 & 28.0 & 29.5 & 20.4 & 22.1 \\
\hline $\mathrm{Zr}$ & 108 & 84 & 83 & 41 & 41 & 90 & 131 & 107 & 92 & 92 & 159 & 69 & 67 \\
\hline $\mathrm{Nb}$ & 9 & 7 & 7 & 3 & 2 & 3 & 4 & 3 & 2 & 2 & 8 & 4 & 3 \\
\hline $\mathrm{Ba}$ & 160 & 115 & 152 & 89 & 57 & 21 & 58 & 6 & 12 & 53 & 89 & 79 & 53 \\
\hline $\mathrm{Ce}$ & 23 & 23 & 17 & 8 & 18 & 19 & 33 & 31 & 22 & 12 & 30 & 16 & 19 \\
\hline \multicolumn{14}{|l|}{ INNA } \\
\hline $\mathrm{Na}_{2} \mathrm{O}$ & 2.72 & 2.50 & 1.96 & 1.94 & 2.27 & 2.92 & 3.25 & 3.21 & 3.03 & 2.73 & 3.26 & 3.44 & 3.16 \\
\hline $\mathrm{Fe}_{2} \mathrm{O}_{3} 7$ day & 7.66 & 8.52 & 8.78 & 9.31 & 9.25 & 11.13 & 8.50 & 8.37 & 9.95 & 10.12 & 6.70 & 7.41 & 7.52 \\
\hline $\mathrm{Fe}_{2} \mathrm{O}_{3} 40$ day & 7.54 & 8.63 & 8.96 & 9.23 & 9.20 & 11.13 & 8.49 & 8.40 & 9.87 & 9.98 & 6.79 & 7.42 & 7.44 \\
\hline $\mathrm{Sc}$ & 37.7 & 31.9 & 31.7 & 34.3 & 34.2 & 39.0 & 36.7 & 37.8 & 37.3 & 35.5 & 22.5 & 34.4 & 36.5 \\
\hline $\mathrm{Cr}$ & 324 & 266 & 259 & 460 & 458 & 377 & 314 & 356 & 374 & 343 & 4 & 44 & 54 \\
\hline $\mathrm{Co}$ & 33.3 & 32.5 & 20.5 & 38.4 & 42.3 & 44.1 & 39.2 & 46.4 & 43.1 & 42.2 & 10.7 & 25.6 & 25.1 \\
\hline $\mathrm{Ni}$ & 65 & 94 & 77 & & 150 & 130 & & & & & & 23 & 18 \\
\hline $\mathrm{Zn}$ & 67 & 82 & 75 & 74 & 73 & 81 & 78 & 86 & 84 & 72 & 93 & 87 & 76 \\
\hline $\mathrm{Rb}$ & 3.3 & 8.6 & 20.1 & 11 & 5.3 & & 3 & 4 & 2 & 1 & 2 & 4.3 & 4.6 \\
\hline $\mathrm{Sr}$ & & 227 & 85 & 117 & 227 & 168 & 236 & 183 & 165 & 165 & 326 & 217 & 287 \\
\hline $\mathrm{Ba}$ & 135 & 119 & 153 & 70.7 & 55 & 34 & 66 & 44 & 26 & 67 & 103 & 62 & 74 \\
\hline $\mathrm{La}$ & 8.69 & 7.27 & 6.99 & 3.58 & 3.72 & 4.45 & 7.43 & 4.56 & 4.38 & 4.23 & 8.57 & 5.91 & 6.32 \\
\hline $\mathrm{Ce}$ & 20.0 & 16.6 & 18.0 & 8.17 & 9.0 & 12.1 & 20.2 & 14.8 & 13.2 & 13.5 & 28.4 & 13.0 & 14.2 \\
\hline Nd & 10.0 & 9.0 & 8.3 & 5.5 & 5.2 & 9.1 & 12.9 & 10.2 & 8.8 & 10.3 & 17.8 & 7.9 & 8.9 \\
\hline $\mathrm{Sm}$ & 3.24 & 2.61 & 2.53 & 1.72 & 1.88 & 3.37 & 3.94 & 3.89 & 3.62 & 3.50 & 4.89 & 2.40 & 2.63 \\
\hline $\mathrm{Eu}$ & 1.14 & 0.90 & 0.94 & 0.77 & 0.82 & 1.29 & 1.29 & 1.39 & 1.32 & 1.29 & 1.65 & 0.96 & 0.99 \\
\hline $\mathrm{Tb}$ & 0.612 & 0.444 & 0.481 & 0.303 & 0.379 & 0.714 & 0.698 & 0.810 & 0.764 & 0.705 & 0.857 & 0.481 & 0.490 \\
\hline $\mathrm{Yb}$ & 2.00 & 1.65 & 1.46 & 1.11 & 1.33 & 2.95 & 2.90 & 3.37 & 2.80 & 2.68 & 3.92 & 2.04 & 2.18 \\
\hline $\mathrm{Lu}$ & 0.323 & 0.262 & 0.226 & 0.190 & 0.213 & 0.465 & 0.408 & 0.470 & 0.434 & 0.443 & 0.608 & 0.319 & 0.381 \\
\hline $\mathrm{Hf}$ & 2.09 & 2.02 & 1.92 & 1.01 & 1.00 & 2.23 & 2.58 & 2.51 & 2.39 & 2.31 & 3.40 & 1.48 & 1.33 \\
\hline $\mathrm{Ta}$ & 0.476 & 0.396 & 0.397 & 0.176 & 0.193 & 0.145 & 0.303 & 0.143 & 0.211 & 0.200 & 0.390 & 0.209 & 0.192 \\
\hline $\mathrm{Au}(\mathrm{ppb})$ & 4.03 & 5.68 & 10.30 & 1.22 & 5.10 & 8.26 & 0.60 & 0.04 & 1.89 & 1.45 & 1.16 & 8.80 & 6.20 \\
\hline Th & 1.03 & 0.84 & 0.82 & 0.28 & 0.27 & 0.27 & 0.52 & 0.23 & 0.19 & 0.28 & 1.18 & 0.44 & 0.45 \\
\hline $\mathrm{U}$ & 0.18 & 0.16 & 0.21 & 0.08 & 0.12 & 0.17 & 0.18 & 0.14 & 0.03 & 0.01 & 0.52 & 0.11 & 0.12 \\
\hline $\mathrm{Fe} / \mathrm{Mn}$ & 46 & 74 & 98 & 68 & 58 & 58 & 35 & 29 & 48 & 46 & 48 & 32 & 32 \\
\hline $\mathrm{Ti} / \mathrm{Zr}$ & 73.3 & 82.8 & 82.3 & 176.9 & 162.3 & 100.6 & 74.6 & 93.6 & 98.4 & 96.4 & 37.3 & 86.9 & 88.6 \\
\hline $\mathrm{Zr} / \mathrm{Y}$ & 4.70 & 5.00 & 6.01 & 3.36 & 2.56 & 2.90 & 4.52 & 3.24 & 3.17 & 3.29 & 5.39 & 3.38 & 3.03 \\
\hline$(\mathrm{Nb} / \mathrm{Zr})_{\mathrm{N}}$ & 1.13 & 1.10 & 1.22 & 1.11 & 0.69 & 0.47 & 0.43 & 0.40 & 0.31 & 0.31 & 0.69 & 0.76 & 0.62 \\
\hline$(\mathrm{Ta} / \mathrm{Hf})_{\mathrm{N}}$ & 1.77 & 1.53 & 1.61 & 1.36 & 1.51 & 0.51 & 0.91 & 0.44 & 0.37 & 0.67 & 0.89 & 1.10 & 1.12 \\
\hline$(\mathrm{La} / \mathrm{Sm})_{\mathrm{N}}$ & 1.63 & 1.70 & 1.68 & 1.27 & 1.21 & 0.81 & 1.15 & 0.71 & 0.74 & 0.74 & 1.07 & 1.50 & 1.46 \\
\hline$(\mathrm{La} / \mathrm{Yb})_{\mathrm{N}}$ & 2.86 & 2.90 & 3.15 & 2.13 & 1.84 & 0.99 & 1.69 & 0.89 & 1.03 & 1.04 & 1.44 & 1.91 & 1.91 \\
\hline $\mathrm{Ce} / \mathrm{Ta}$ & 42 & 42 & 45 & 46 & 47 & 30 & 67 & 103 & 63 & 68 & 73 & 62 & 74 \\
\hline $\mathrm{Eu} / \mathrm{Eu}^{*}$ & 1.01 & 1.02 & 1.03 & 1.14 & 1.10 & 1.03 & 0.99 & 1.00 & 1.01 & 1.02 & 1.00 & 1.06 & 1.04 \\
\hline
\end{tabular}


Table 1 (continued).

\begin{tabular}{|c|c|c|c|c|c|c|c|c|c|c|c|c|c|}
\hline Hole & $795 B$ & $797 \mathrm{C}$ & $797 \mathrm{C}$ & $797 \mathrm{C}$ & $797 \mathrm{C}$ & 797C & $797 \mathrm{C}$ & $797 \mathrm{C}$ & $797 \mathrm{C}$ & $797 \mathrm{C}$ & $797 \mathrm{C}$ & $797 \mathrm{C}$ & $797 \mathrm{C}$ \\
\hline Section & $40 \mathrm{R}-2$ & $8 \mathrm{R}-2$ & IOR-1 & IIR-1 & $12 \mathrm{R}-2$ & 13R-2 & $14 \mathrm{R}-1$ & $15 R-1$ & 19R-2 & 19R-4 & 20R-2 & 2IR-I & $24 R-6$ \\
\hline XRF Interval & $123-125$ & $126-130$ & $90-92$ & $72-74$ & $81-83$ & $72-73$ & $70-72$ & $77-79$ & $42-44$ & $61-64$ & 104-108 & $82-85$ & $38-40$ \\
\hline INNA Interval & $116-121$ & $127-130^{*}$ & $89-91$ & $73-75$ & $72-74$ & $63-65$ & $65-67$ & $79-81^{*}$ & $61-63 *$ & $57-60^{*}$ & $98-101 *$ & $80-84 *$ & $41-45$ \\
\hline Unit & 3B & 1 & 2 & 3 & 3 & 3 & 4 & 5 & 7 & 7 & 8 & 8 & 9 \\
\hline \multicolumn{14}{|l|}{ XRF } \\
\hline $\mathrm{SiO}_{2}$ & 54.82 & 49.81 & 50.16 & 50.63 & 47.33 & 48.67 & 48.36 & 48.99 & 49.14 & 45.37 & 46.39 & 47.86 & 47.98 \\
\hline $\mathrm{TiO}_{2}$ & 0.93 & 1.27 & 1.12 & 1.13 & 0.95 & 0.98 & 1.24 & 1.22 & 0.88 & 1.06 & 1.10 & 0.88 & 0.92 \\
\hline $\mathrm{Al}_{2} \mathrm{O}_{3}$ & 17.79 & 21.17 & 19.08 & 19.49 & 17.61 & 18.26 & 20.96 & 21.11 & 17.97 & 20.69 & 20.53 & 18.06 & 17.40 \\
\hline $\mathrm{Fe}_{2} \mathrm{O}_{3}$ & 7.73 & 6.98 & 7.50 & 6.26 & 8.29 & 8.04 & 7.76 & 7.22 & 7.19 & 7.88 & 9.11 & 7.83 & 8.52 \\
\hline $\mathrm{MnO}$ & 0.17 & 0.21 & 0.41 & 0.08 & 0.17 & 0.31 & 0.43 & 0.37 & 0.28 & 0.74 & 0.45 & 0.19 & 0.18 \\
\hline $\mathrm{MgO}$ & 5.83 & 7.25 & 6.21 & 10.63 & 9.76 & 7.48 & 8.96 & 9.05 & 8.12 & 8.41 & 12.65 & 8.80 & 8.75 \\
\hline $\mathrm{CaO}$ & 8.92 & 7.51 & 10.85 & 6.34 & 11.13 & 11.70 & $\quad 6.17$ & 7.09 & 11.57 & 10.45 & 4.60 & 10.30 & 11.59 \\
\hline $\mathrm{Na}_{2} \mathrm{O}$ & 3.36 & 4.32 & 3.75 & 3.55 & 3.15 & 3.28 & 3.86 & 3.67 & 3.09 & 3.55 & 2.81 & 3.40 & 2.80 \\
\hline $\mathrm{K}_{2} \mathrm{O}$ & 0.22 & 0.32 & 0.11 & 1.03 & 0.06 & 0.04 & 0.17 & 0.18 & 0.07 & 0.08 & 0.40 & 0.13 & 0.12 \\
\hline $\mathrm{P}_{2} \mathrm{O}_{5}$ & 0.34 & 0.17 & 0.14 & 0.12 & 0.11 & 0.10 & 0.12 & 0.12 & 0.08 & 0.09 & 0.10 & 0.09 & 0.10 \\
\hline Total & 100.11 & 99.01 & 99.33 & 99.26 & 98.56 & 98.86 & 98.03 & 99.02 & 98.39 & 98.32 & 98.14 & 97.54 & 98.36 \\
\hline LOI & 3.49 & 4.16 & 2.39 & 4.78 & 1.94 & 2.33 & 5.26 & 5.02 & 2.40 & 7.86 & 8.44 & 4.17 & 2.12 \\
\hline $\mathrm{Mg} \#$ & 0.62 & 0.70 & 0.65 & 0.79 & 0.72 & 0.67 & 0.72 & 0.73 & 0.71 & 0.70 & 0.75 & 0.71 & 0.69 \\
\hline $\mathrm{Cr}$ & 19 & 315 & 272 & 277 & 216 & 245 & 323 & 325 & 317 & 396 & 314 & 272 & 293 \\
\hline $\mathrm{Ni}$ & 24 & 143 & 150 & 160 & 163 & 145 & 172 & 140 & 101 & 106 & 153 & 143 & 94 \\
\hline V & 225 & 220 & 214 & 192 & 166 & 166 & 248 & 250 & 200 & 260 & 215 & 183 & 187 \\
\hline $\mathrm{Cu}$ & 43 & 98 & 89 & 92 & 81 & 88 & 110 & 89 & 83 & 86 & 76 & 67 & 73 \\
\hline $\mathrm{Zn}$ & 91 & 95 & 63 & 40 & 53 & 59 & 89 & 96 & 61 & 65 & 68 & 59 & 57 \\
\hline $\mathrm{Rb}$ & 5.3 & 3 & 1.0 & 8.0 & 1.0 & & 1.0 & 2 & 0 & 1 & 9 & 1 & 1.0 \\
\hline $\mathrm{Sr}$ & 223 & 314 & 302 & 242 & 239 & 229 & 229 & 252 & 179 & 189 & 138 & 189 & 218 \\
\hline $\mathrm{Y}$ & 27.5 & 22.0 & 21.0 & 18.0 & 19.0 & 21.0 & 19.0 & 19.0 & 21.0 & 21.0 & 15.0 & 20.0 & 20.0 \\
\hline $\mathrm{Zr}$ & 63 & 110 & 97 & 92 & 77 & 73 & 89 & 87 & 54 & 62 & 67 & 61 & 67 \\
\hline $\mathrm{Nb}$ & 3 & 1 & I & 1 & & & & & & & & 1 & 1 \\
\hline $\mathrm{Ba}$ & & 23 & 25 & 31 & 14 & 11 & 12 & 25 & 17 & 12 & 15 & 26 & 4 \\
\hline $\mathrm{Ce}$ & 27 & 16 & 13 & 19 & 10 & 6 & 15 & 9 & 9 & 14 & 15 & 5 & 10 \\
\hline INNA & & & & & & & & & & 3.20 & 2.72 & 3.01 & 2.73 \\
\hline $\mathrm{Na}_{2} \mathrm{O}$ & 2.88 & 3.57 & 3.21 & 2.88 & 2.92 & 2.91 & 3.34 & 3.43 & 3.14 & 6.37 & 7.43 & 7.76 & 8.45 \\
\hline $\mathrm{Fe}_{2} \mathrm{O}_{3} 7$ day & 7.93 & 6.80 & 7.36 & 6.16 & 8.06 & 6.96 & 6.40 & 7.19 & 7.67 & 6.42 & 7.39 & 7.82 & 8.53 \\
\hline $\mathrm{Fe}_{2} \mathrm{O}_{3} 40$ day & 7.95 & 6.74 & 7.55 & 6.16 & 8.01 & 7.18 & 6.40 & 7.23 & 7.66 & 36.7 & 36.9 & 32.5 & 33.2 \\
\hline $\mathrm{Sc}$ & 32.2 & 35.4 & 32.8 & 30.2 & 31.3 & 31.7 & 37.0 & 37.4 & 36.3 & 401 & 381 & 384 & 362 \\
\hline $\mathrm{Cr}$ & 42 & 321 & 297 & 320 & 290 & 309 & 365 & 345 & 391 & 41.3 & 46.9 & 41.3 & 39.2 \\
\hline $\mathrm{Co}$ & 22.9 & 47.3 & 43.3 & 50.5 & 42.3 & 42.4 & 52.2 & 52.9 & 43.7 & & & & 80 \\
\hline $\mathrm{Ni}$ & 14 & & 127 & 140 & 146 & 119 & 148 & & & 75 & 94 & 69 & 53 \\
\hline $\mathrm{Zn}$ & 84 & 94 & 59 & 35 & 58 & 56 & 66 & 98 & 63 & & 4 & 1 & 1.0 \\
\hline $\mathrm{Rb}$ & 9.0 & 2 & 1.9 & 7.3 & 0.6 & 2.2 & 1.0 & 2 & & 179 & 110 & 144 & 229 \\
\hline $\mathrm{Sr}$ & 174 & 291 & 272 & 206 & 225 & 231 & 201 & 216 & 146 & 5 & 19 & 29 & 14 \\
\hline $\mathrm{Ba}$ & 46 & 17 & 10 & 44 & 27 & 17 & 27 & 11 & 8 & 3.15 & 2.73 & 2.01 & 2.82 \\
\hline $\mathrm{La}$ & 6.38 & 4.20 & 3.51 & 3.39 & 3.12 & 2.49 & 4.23 & 2.68 & 1.51 & 7.0 & 8.8 & 5.4 & 8.8 \\
\hline $\mathrm{Ce}$ & 14.8 & 13.6 & 11.5 & 10.7 & 8.9 & 8.0 & 11.7 & 9.5 & 5.6 & 6.2 & 6.2 & 5.0 & 6.3 \\
\hline $\mathrm{Nd}$ & 8.0 & 10.4 & 7.7 & 6.9 & 7.7 & 6.8 & 8.8 & 7.4 & 6.3 & 2.53 & 2.55 & 1.99 & 2.33 \\
\hline $\mathrm{Sm}$ & 2.56 & 2.90 & 2.64 & 2.77 & 2.53 & 2.33 & 2.79 & 2.70 & 2.52 & 1.03 & 0.99 & 0.82 & 0.94 \\
\hline $\mathrm{Eu}$ & 0.98 & 1.13 & 1.03 & 1.05 & 0.97 & 0.96 & 1.15 & 1.08 & 0.99 & 0.495 & 0.499 & 0.438 & 0.543 \\
\hline $\mathrm{Tb}$ & 0.481 & 0.569 & 0.567 & 0.526 & 0.514 & 0.489 & 0.526 & 0.502 & 0.510 & 2.11 & 1.81 & 1.80 & 1.96 \\
\hline $\mathrm{Yb}$ & 2.22 & 2.01 & 2.12 & 1.30 & 2.03 & 2.04 & 1.76 & 1.87 & 2.27 & 0.292 & 0.255 & 0.295 & 0.310 \\
\hline Lu & 0.345 & 0.306 & 0.315 & 0.202 & 0.337 & 0.303 & 0.286 & 0.286 & 0.333 & 1.43 & 1.64 & 1.31 & 1.42 \\
\hline Hf & 1.30 & 2.29 & 1.93 & 1.84 & 1.72 & 1.64 & 1.86 & 1.95 & 1.50 & 0.090 & 0.069 & 0.105 & 0.083 \\
\hline Ta & 0.167 & 0.131 & 0.120 & 0.069 & 0.059 & 0.013 & 0.019 & 0.065 & 0.030 & 0.45 & 1.00 & 0.04 & 6.30 \\
\hline $\mathrm{Au}(\mathrm{ppb})$ & 8.60 & 1.76 & 9.30 & 8.20 & 5.90 & 8.70 & 5.50 & 1.57 & 0.31 & 0.12 & 0.10 & & 0.09 \\
\hline Th & 0.37 & 0.08 & 0.13 & 0.10 & 0.09 & & 0.12 & 6.60 & 0.07 & 0.05 & 0.09 & 0.03 & 0.10 \\
\hline $\mathrm{U}$ & 0.19 & 0.08 & 0.01 & 0.09 & 0.12 & & & 0.09 & 0.10 & & & & \\
\hline $\mathrm{Fe} / \mathrm{Mn}$ & 41 & 30 & 17 & 71 & 44 & 23 & 16 & 18 & 23 & 10 & 18 & 37 & 43 \\
\hline $\mathrm{Ti} / \mathrm{Zr}$ & 88.5 & 69.2 & 69.2 & 73.6 & 74.0 & 80.5 & 83.5 & 84.1 & 97.7 & 102.5 & 98.4 & 86.5 & 82.3 \\
\hline $\mathrm{Zr} / \mathrm{Y}$ & 2.29 & 5.00 & 4.62 & 5.11 & 4.05 & 3.48 & 4.68 & 4.58 & 2.57 & 2.95 & 4.47 & 3.05 & 3.35 \\
\hline$(\mathrm{Nb} / \mathrm{Zr})_{\mathrm{N}}$ & 0.63 & 0.13 & 0.15 & 0.15 & & & & & & & & 0.23 & 0.21 \\
\hline$(\mathrm{Ta} / \mathrm{Hf})_{\mathrm{N}}$ & 1.00 & 0.45 & 0.48 & 0.29 & 0.27 & 0.06 & 0.08 & 0.26 & 0.16 & 0.49 & 0.33 & 0.62 & 0.46 \\
\hline$(\mathrm{La} / \mathrm{Sm})_{\mathrm{N}}$ & 1.52 & 0.88 & 0.81 & 0.75 & 0.75 & 0.65 & 0.92 & 0.61 & 0.37 & 0.76 & 0.65 & 0.62 & 0.74 \\
\hline$(\mathrm{La} / \mathrm{Yb})_{\mathrm{N}}$ & 1.89 & 1.38 & 1.09 & 1.72 & 1.01 & 0.80 & 1.58 & 0.94 & 0.44 & 0.98 & 0.99 & 0.74 & 0.95 \\
\hline $\mathrm{Ce} / \mathrm{Ta}$ & 89 & 104 & 96 & 155 & 150 & 613 & 616 & 145 & 187 & 78 & 127 & 52 & 106 \\
\hline $\mathrm{Eu} / \mathrm{Eu}^{*}$ & 1.04 & 1.05 & 1.03 & 1.04 & 1.04 & 1.06 & 1.07 & 10.6 & 1.04 & 1.06 & 1.05 & 1.06 & 1.04 \\
\hline
\end{tabular}


Table 1 (continued).

\begin{tabular}{|c|c|c|c|c|c|c|c|c|c|c|c|c|c|}
\hline Hole & $797 \mathrm{C}$ & $797 \mathrm{C}$ & $797 \mathrm{C}$ & $797 \mathrm{C}$ & $797 \mathrm{C}$ & $797 \mathrm{C}$ & $797 \mathrm{C}$ & $797 \mathrm{C}$ & $797 \mathrm{C}$ & $797 \mathrm{C}$ & $797 \mathrm{C}$ & $797 \mathrm{C}$ & $797 \mathrm{C}$ \\
\hline Section & 26R-1 & $27 \mathrm{R}-1$ & $28 \mathrm{R}-1$ & 29R-1 & $31 \mathrm{R}-2$ & $32 R-2$ & $33 R-1$ & $33 R-4$ & $36 \mathrm{R}-2$ & $36 \mathrm{R}-3$ & $41 R-1$ & $44 R-3$ & $45 \mathrm{R}-1$ \\
\hline XRF Interval & $76-78$ & $30-32$ & $49-51$ & $69-70$ & $36-38$ & $15-17$ & $63-65$ & $5-7$ & $12-14$ & $2-4$ & $23-26$ & $15-18$ & \\
\hline INNA Interval & $81-83$ & $23-26^{*}$ & $45-47$ & $50-53$ & $30-33$ & $18-20 *$ & $72-76^{*}$ & $3-5$ & $12-14^{*}$ & $2-4^{*}$ & $23-26 *$ & $15-18^{*}$ & $81-86$ \\
\hline Unit & 10 & 11 & 12 & 12 & 13 & 14 & 14 & 15 & 16 & 17 & 19 & 20 & 21 \\
\hline \multicolumn{14}{|l|}{ XRF } \\
\hline $\mathrm{SiO}_{2}$ & 51.43 & 49.35 & 52.53 & 52.31 & 50.24 & 49.16 & 52.10 & & & & & & \\
\hline $\mathrm{TiO}_{2}^{2}$ & 2.99 & 1.87 & 2.18 & 2.02 & 1.65 & 1.75 & 2.00 & 2.01 & & & & & \\
\hline $\mathrm{Al}_{2} \mathrm{O}_{3}$ & 15.03 & 15.91 & 14.34 & 14.62 & 14.91 & 16.51 & 15.59 & & & & & & \\
\hline $\mathrm{Fe}_{2} \mathrm{O}_{3}$ & 11.63 & 10.73 & 10.51 & 10.40 & 10.28 & 9.96 & 11.51 & & & & & & \\
\hline $\mathrm{MnO}$ & 0.32 & 0.20 & 0.18 & 0.18 & 0.19 & 0.31 & 0.49 & & & & & & \\
\hline $\mathrm{MgO}$ & 5.64 & 7.85 & 6.19 & 5.89 & 6.13 & 7.10 & 8.52 & & & & & & \\
\hline $\mathrm{CaO}$ & 3.88 & 6.57 & 5.27 & 6.53 & 10.04 & 8.78 & 1.35 & & & & & & \\
\hline $\mathrm{Na}_{2} \mathrm{O}$ & 4.48 & 3.90 & 4.42 & 4.14 & 3.55 & 3.72 & 3.75 & & & & & & \\
\hline $\mathrm{K}_{2} \mathrm{O}$ & 1.03 & 0.38 & 1.17 & 1.07 & 0.52 & 0.48 & 2.42 & & & & & & \\
\hline $\mathrm{P}_{2} \mathrm{O}_{5}$ & 0.43 & 0.25 & 0.33 & 0.29 & 0.24 & 0.24 & 0.33 & & & & & & \\
\hline Total & 96.86 & 97.01 & 97.12 & 97.45 & 97.75 & 98.01 & 98.06 & & & & & & \\
\hline LOI & 3.06 & 3.42 & 2.53 & 2.08 & 1.64 & 2.54 & 4.37 & & & & & & \\
\hline Mg\# & 0.52 & 0.62 & 0.56 & 0.56 & 0.57 & 0.61 & 0.62 & & & & & & \\
\hline $\mathrm{Cr}$ & 14 & 169 & 11 & 26 & 134 & 195 & 85 & 87 & & & & & \\
\hline $\mathrm{Ni}$ & 11 & 39 & 14 & 18 & 36 & 60 & 29 & 31 & & & & & \\
\hline V & 409 & 328 & 306 & 303 & 266 & 293 & 283 & 304 & & & & & \\
\hline $\mathrm{Cu}$ & 39 & 39 & 46 & 45 & 59 & 60 & 42 & 50 & & & & & \\
\hline $\mathrm{Zn}$ & 126 & 62 & 109 & 99 & 87 & 69 & 92 & 86 & & & & & \\
\hline $\mathrm{Rb}$ & 7.0 & 2 & 15.0 & 14.0 & 3.0 & 2 & 32 & 11.0 & & & & & \\
\hline $\mathrm{Sr}$ & 305 & 298 & 265 & 275 & 276 & 329 & 110 & 295 & & & & & \\
\hline $\mathrm{Y}$ & 53.0 & 39.0 & 45.0 & 39.0 & 34.0 & 33.0 & 38.0 & 37.0 & & & & & \\
\hline $\mathrm{Zr}$ & 283 & 162 & 231 & 207 & 160 & 159 & 224 & 180 & & & & & \\
\hline $\mathrm{Nb}$ & 11 & 6 & 8 & 8 & 6 & 6 & 9 & 7 & & & & & \\
\hline $\mathrm{Ba}$ & 217 & 127 & 242 & 242 & 181 & 145 & 282 & 159 & & & & & \\
\hline $\mathrm{Ce}$ & 57 & 31 & 41 & 38 & 40 & 32 & 44 & 43 & & & & & \\
\hline \multicolumn{14}{|l|}{ INNA } \\
\hline $\mathrm{Na}_{2} \mathrm{O}$ & 4.17 & 3.46 & 3.73 & 3.70 & 3.35 & 3.36 & 4.18 & 3.39 & 3.32 & 3.93 & 2.97 & 4.19 & 2.55 \\
\hline $\mathrm{Fe}_{2} \mathrm{O}_{3} 7$ day & 11.29 & 9.78 & 9.94 & 10.07 & 9.97 & 9.70 & 10.74 & 10.35 & 10.41 & 11.56 & 10.87 & 10.36 & 10.57 \\
\hline $\mathrm{Fe}_{2} \mathrm{O}_{3} 40$ day & 11.21 & 9.77 & 10.02 & 10.05 & 9.91 & 9.69 & 10.71 & 10.39 & 10.56 & 11.48 & 10.93 & 10.35 & 10.57 \\
\hline $\mathrm{Sc}$ & 33.3 & 37.0 & 30.9 & 32.1 & 36.7 & 38.2 & 34.0 & 34.3 & 46.3 & 44.1 & 41.4 & 28.2 & 39.3 \\
\hline $\mathrm{Cr}$ & 33 & 198 & 33 & 44 & 197 & 228 & 95 & 125 & 296 & 294 & 174 & 32 & 130 \\
\hline Co & 38.4 & 32.0 & 27.5 & 24.7 & 33.8 & 36.0 & 34.3 & 29.6 & 34.1 & 40.9 & 36.0 & 26.3 & 33.9 \\
\hline $\mathrm{Ni}$ & 27 & & & 6 & 35 & & & 19 & & & & & 22 \\
\hline $\mathrm{Zn}$ & 113 & 92 & 114 & 94 & 79 & 86 & 121 & 88 & 130 & 160 & 94 & 84 & 86 \\
\hline $\mathrm{Rb}$ & 9.4 & 4 & 13.4 & 12.7 & 5.6 & & 32 & 13.5 & 3 & 2 & 5 & 19 & 7.1 \\
\hline $\mathrm{Sr}$ & 297 & 292 & 259 & 285 & 247 & 281 & 67 & 317 & 92 & 95 & 222 & 104 & 197 \\
\hline $\mathrm{Ba}$ & 220 & 72 & 182 & 203 & 88 & 111 & 448 & 145 & 44 & 70 & 98 & 212 & 96 \\
\hline $\mathrm{La}$ & 20.6 & 9.24 & 16.5 & 16.0 & 11.0 & 10.49 & 16.7 & 14.0 & 9.03 & 8.21 & 9.54 & 16.06 & 9.79 \\
\hline $\mathrm{Ce}$ & 48.8 & 25.1 & 40.2 & 39.2 & 29.5 & 27.3 & 42.4 & 34.7 & 24.9 & 21.6 & 23.3 & 42.2 & 25.9 \\
\hline $\mathrm{Nd}$ & 28.1 & 14.8 & 21.8 & 19.6 & 13.8 & 16.0 & 20.7 & 18.0 & 16.1 & 13.6 & 15.1 & 23.9 & 16.9 \\
\hline $\mathrm{Sm}$ & 7.74 & 4.62 & 6.16 & 6.06 & 4.63 & 4.51 & 5.79 & 5.25 & 4.92 & 4.12 & 5.29 & 6.63 & 5.25 \\
\hline $\mathrm{Eu}$ & 2.24 & 1.55 & 1.77 & 1.73 & 1.54 & 1.54 & 1.31 & 1.60 & 1.87 & 1.52 & 1.64 & 1.92 & 1.70 \\
\hline $\mathrm{Tb}$ & 1.38 & 0.885 & 1.10 & 1.08 & 1.00 & 0.775 & 1.09 & 0.980 & 0.954 & 0.860 & 0.982 & 1.16 & 1.05 \\
\hline $\mathrm{Yb}$ & 4.79 & 3.60 & 4.27 & 3.89 & 3.45 & 2.90 & 3.58 & 3.70 & 3.90 & 3.65 & 4.15 & 4.35 & 3.88 \\
\hline $\mathrm{Lu}$ & 0.750 & 0.553 & 0.601 & 0.593 & 0.538 & 0.521 & 0.536 & 0.531 & 0.649 & 0.522 & 0.593 & 0.671 & 0.637 \\
\hline $\mathrm{Hf}$ & 5.90 & 3.45 & 4.76 & 4.52 & 3.37 & 3.37 & 4.86 & 4.17 & 3.21 & 3.11 & 3.49 & 5.08 & 3.74 \\
\hline $\mathrm{Ta}$ & 0.707 & 0.426 & 0.581 & 0.536 & 0.419 & 0.407 & 0.711 & 0.483 & 0.427 & 0.333 & 0.387 & 0.652 & 0.410 \\
\hline $\mathrm{Au}(\mathrm{ppb})$ & 7.90 & 0.46 & 9.20 & 9.40 & 9.90 & 0.89 & 0.37 & 10.50 & 1.03 & 0.25 & 0.17 & & 5.50 \\
\hline Th & 2.77 & 1.36 & 2.96 & 2.82 & 1.51 & 1.35 & 2.36 & 1.74 & 0.48 & 0.37 & 0.73 & 2.59 & 0.71 \\
\hline U & 0.58 & 0.38 & 0.80 & 0.68 & 0.50 & 0.26 & 0.70 & 0.44 & 0.18 & 0.22 & 0.30 & 0.47 & 0.31 \\
\hline $\mathrm{Fe} / \mathrm{Mn}$ & 33 & 48 & 53 & 52 & 49 & 29 & 21 & & & & & & \\
\hline $\mathrm{Ti} / \mathrm{Zr}$ & 63.3 & 69.2 & 56.6 & 58.5 & 61.8 & 66.0 & 53.5 & 66.9 & & & & & \\
\hline $\mathrm{Zr} / \mathrm{Y}$ & 5.34 & 4.15 & 5.13 & 5.31 & 4.71 & 4.82 & 5.89 & 4.86 & & & & & \\
\hline$(\mathrm{Nb} / \mathrm{Zr})_{\mathrm{N}}$ & 0.55 & 0.53 & 0.49 & 0.55 & 0.53 & 0.54 & 0.57 & 0.55 & & & & & \\
\hline$(\mathrm{Ta} / \mathrm{Hf})_{\mathrm{N}}$ & 0.93 & 0.96 & 0.95 & 0.92 & 0.97 & 0.94 & 1.14 & 0.90 & 1.04 & 0.83 & 0.86 & 1.00 & 0.85 \\
\hline$(\mathrm{La} / \mathrm{Sm})_{\mathrm{N}}$ & 1.62 & 1.22 & 1.63 & 1.61 & 1.45 & 1.42 & 1.76 & 1.63 & 1.12 & 1.21 & 1.10 & 1.48 & 1.14 \\
\hline$(\mathrm{La} / \mathrm{Yb})_{\mathrm{N}}$ & 2.83 & 1.69 & 2.55 & 2.71 & 2.10 & 2.38 & 3.07 & 2.49 & 1.53 & 1.48 & 1.51 & 2.43 & 1.66 \\
\hline $\mathrm{Ce} / \mathrm{Ta}$ & 69 & 59 & 69 & 73 & 70 & 67 & 60 & 72 & 58 & 65 & 60 & 65 & 63 \\
\hline $\mathrm{Eu} / \mathrm{Eu}^{*}$ & 0.96 & 0.99 & 0.95 & 0.95 & 0.98 & 1.01 & 0.87 & 0.96 & 1.03 & 1.01 & 0.97 & 0.96 & 0.98 \\
\hline
\end{tabular}

Note: Interval refers to core interval (in centimeters) from which the sample was taken. All trace elements are in ppm exept for Au, which is in ppb. Methods and estimated errors for the shipboard X-Ray Fluorescence (XRF) data are discussed in Tamaki, Pisciotto, Allan, et al., 1990; D. Sims was the analyst. Methods for the shore-based neutron activation analyses (INAA) are discussed in the text; M. Gorton was the analyst. Estimated counting errors (in ppm unless given otherwise) for the INAA are illustrated by giving them for Sample 127-797C-28R-1, 45-47 cm: $\mathrm{Na}_{2} \mathrm{O}, 0.009 \% ; 7$-day $\mathrm{Fe}_{2} \mathrm{O}_{3}, 0.01 \% ; 40$-day $\mathrm{Fe}_{2} \mathrm{O}_{3}, 0.02 \% ; \mathrm{Sc}, 0.02 \% ; \mathrm{Cr}, 1 ; \mathrm{Co}, 0.1 \% ; \mathrm{Ni}, 7$ (a typical figure for all samples); $\mathrm{Zn}, 2 ; \mathrm{Rb}$, $0.7 ; \mathrm{Sr}, 14 ; \mathrm{Ba}, 6 ; \mathrm{La}, 0.06 ; \mathrm{Ce}, 0.3 ; \mathrm{Nd}, 0.3 ; \mathrm{Sm}, 0.007 ; \mathrm{Eu}, 0.01 ; \mathrm{Tb}, 0.015 ; \mathrm{Yb}, 0.025 ; \mathrm{Lu}, 0.004 ; \mathrm{Hf}, 0.05 ; \mathrm{Ta}, 0.01 ; \mathrm{Au}, 0.1 \mathrm{ppb} ; \mathrm{Th}, 0.02 ;$ and U, 0.02. LOI = loss on ignition; $\mathrm{Mg} \#=\mathrm{Mg} /\left(\mathrm{Mg}+\mathrm{Fe}^{2+}\right)$; and $\mathrm{Nb} / \mathrm{Zr}, \mathrm{Ta} / \mathrm{Hf}, \mathrm{La} / \mathrm{Sm}$, and $\mathrm{La} / \mathrm{Yb}$ ratios are chondrite-normalized, with chondritic values for $\mathrm{Nb}$ and $\mathrm{Zr}$ from Erlank and $\mathrm{Kable}(1976), \mathrm{Ta}$ and Hf from Anders and Ebihara (1982), and La, Sm, and $\mathrm{Yb}$ from Matsuda et al., 1973. Eu/Eu* refers to observed Eu/expected Eu, where the expected Eu is calculated using the "effective" ionic radii of trivalent REE in sixfold coordination (Shannon, 1976). Comparisons of the XRF and INAA data sets should take into account that the XRF samples were run on ignited samples, whereas the INAA samples were run on unignited samples. Samples with asterisks are corrected for weighing errors as explained in text. As a result, element abundances are slightly less precise than for other samples, precision being degraded by a few percent. 
Table 2. Shore-based X-ray fluorescence (XRF), whole-rock data for units not run onboard ship due to time constraints. Methods are discussed in the text.

\begin{tabular}{|c|c|c|c|c|c|c|c|c|}
\hline Hole & $797 \mathrm{C}$ & $797 \mathrm{C}$ & $797 \mathrm{C}$ & $797 \mathrm{C}$ & $797 \mathrm{C}$ & $797 \mathrm{C}$ & $797 \mathrm{C}$ & $797 \mathrm{C}$ \\
\hline Section & $33 R-4$ & $34 R-1$ & $36 \mathrm{R}-2$ & $36 \mathrm{R}-3$ & $36 \mathrm{R}-3$ & 4IR-1 & $44 R-3$ & $45 \mathrm{R}-1$ \\
\hline Interval $(\mathrm{cm})$ & $3-5$ & $40-43$ & $4-7$ & $2-4$ & $12-14$ & $23-26$ & $15-18$ & $81-86$ \\
\hline Unit & 15 & 15 & 16 & 17 & 18 & 19 & 20 & 21 \\
\hline $\mathrm{SiO}_{2}$ & 52.63 & 50.56 & 47.75 & 49.27 & 52.40 & 51.29 & 53.95 & 51.69 \\
\hline $\mathrm{TiO}_{2}$ & 2.02 & 1.93 & 1.96 & 1.79 & 1.68 & 2.02 & 2.09 & 2.10 \\
\hline $\mathrm{Al}_{2} \mathrm{O}_{3}$ & 15.19 & 15.71 & 18.76 & 17.33 & 16.45 & 15.41 & 14.06 & 4.61 \\
\hline $\mathrm{Fe}_{2} \mathrm{O}_{3}$ & 9.96 & 9.98 & 10.31 & 11.05 & 11.77 & 10.13 & 10.13 & 10.39 \\
\hline $\mathrm{MnO}^{2}$ & 0.25 & 0.52 & 0.41 & 0.45 & 0.37 & 0.34 & 0.27 & 0.24 \\
\hline $\mathrm{MgO}$ & 6.79 & 6.33 & 10.11 & 11.65 & 9.94 & 8.10 & 6.28 & 7.52 \\
\hline $\mathrm{CaO}$ & 7.77 & 9.47 & 5.37 & 3.13 & 2.18 & 8.10 & 5.06 & 8.77 \\
\hline $\mathrm{Na}_{2} \mathrm{O}$ & 3.69 & 3.55 & 3.73 & 3.58 & 3.65 & 3.23 & 4.78 & 2.87 \\
\hline $\mathrm{K}_{2} \mathrm{O}$ & 0.81 & 0.72 & 0.33 & 0.38 & 0.42 & 0.43 & 1.68 & 0.81 \\
\hline $\mathrm{P}_{2} \mathrm{O}_{5}$ & 0.28 & 0.27 & 0.24 & 0.21 & 0.20 & 0.27 & 0.36 & 0.26 \\
\hline Total & 100.50 & 100.15 & 100.11 & 100.07 & 100.37 & 100.45 & 100.32 & 100.41 \\
\hline $\mathrm{Mg \#}$ & 0.60 & 0.58 & 0.68 & 0.70 & 0.65 & 0.64 & 0.58 & 0.61 \\
\hline LOI & 6.12 & 6.31 & 7.90 & 7.36 & 5.98 & 5.31 & 7.29 & 4.89 \\
\hline $\mathrm{Cr}^{*}$ & 98 & 156 & 218 & 309 & 173 & 144 & 22 & 86 \\
\hline $\mathrm{Ni} *$ & 40 & 50 & 50 & 55 & 48 & 45 & 27 & 42 \\
\hline$V^{*}$ & 363 & 304 & 596 & 480 & 651 & 390 & 506 & 411 \\
\hline $\mathrm{Cu}^{*}$ & 37 & 35 & 62 & 70 & 60 & 42 & 24 & 50 \\
\hline $\mathrm{Zn}^{*}$ & 71 & 74 & 136 & 90 & 123 & 76 & 85 & 81 \\
\hline $\mathrm{Rb}$ & 11 & 8 & 2 & 3 & 3 & 4 & 27 & 9 \\
\hline $\mathrm{Sr}$ & 285 & 290 & 196 & 153 & 120 & 248 & 121 & 239 \\
\hline $\mathrm{Y}$ & 35 & 31 & 41 & 35 & 25 & 38 & 45 & 40 \\
\hline $\mathrm{Zr}^{*}$ & 159 & 138 & 111 & 121 & 105 & 140 & 183 & 146 \\
\hline $\mathrm{Pb}$ & 10 & 5 & 3 & 3 & 4 & 3 & 3 & 4 \\
\hline $\mathrm{Ga}$ & 19 & 20 & 20 & 18 & 18 & 19 & 20 & 20 \\
\hline $\mathrm{Fe} / \mathrm{Mn}$ & 36 & 17 & 23 & 22 & 29 & 27 & 34 & 39 \\
\hline $\mathrm{Ti} / \mathrm{Zr}$ & 76.2 & 83.8 & 105.9 & 88.7 & 95.9 & 86.5 & 68.5 & 86.2 \\
\hline
\end{tabular}

Note: M. Rhodes, University of Massachusetts, was the analyst for the unstarred elements; S. Nelson, Tulane University, was the analyst for the elements with asterisks.

finding provides support for the use of these elements in fresher samples for testing of petrogenetic hypothesis. Further work is needed to better delineate the conditions of apparent elemental mobility exhibited by the altered unit margins, particularly the apparent mobility of the HREE.

\section{GEOCHEMICAL CHARACTERISTICS OF LEG 127/128 IGNEOUS UNITS}

In this section we present the results of our INAA analyses on the Leg 127/128 igneous rocks. Due to our belief that the major-element geochemistry of these units does not accurately reflect that of the original silicate liquids, we will not attempt direct liquid-line-of-descent estimations or calculations. We refer the reader to the shipboard science volumes (Tamaki, Pisciotto, Allan, et al., 1990; Ingle, Suyehiro, von Breymann et al., 1990) and to Thy (this volume) and Yamashita et al. (this volume) for discussions involving the trends exhibited by the major elements. We will instead concentrate on the minor and trace elements, focusing primarily on the elements that exhibited little mobility during alteration. It should be noted that our preliminary studies support the unit boundaries adopted from the reports of both shipboard parties, with the possible exception of Unit 8 defined in Hole 794D (Tamaki, Pisciotto, Allan, et al., 1990; Ingle, Suyehiro, von Breymann et al., 1990).

\section{Site 794, Northern Yamato Basin}

All units are interpreted to be tholeiitic basalts, based on their chemistry and quench-margin (near-liquidus) pre-alteration mineralogy of plagioclase, olivine, and Cr-spinel (Tamaki, Pisciotto, Allan, et al., 1990; Ingle, Suyehiro, von Breymann et al., 1990; Allan et al., 1990). Some units appear to be quite primitive, but caution must be exercised as the $\mathrm{MgO}$ contents have been affected by alteration. Chondrite-normalized (Masuda, 1973) REE compositions of samples from Site 794 are plotted in Figure 15, and selected elements from these samples normalized against normal mid-ocean ridge basalt (N-MORB) abundances (Pearce et al., 1981) are plotted in Figure 16.

Tamaki, Pisciotto, Allan, et al. (1990) and Ingle, Suyehiro, von Breymann et al. (1990) recognized that several spatially and compositionally distinct groups of units occur. Site 794 units were classified by Ingle, Suyehiro, von Breymann et al. (1990) into an upper complex (Units 1 to 5) and a lower complex (Units 7 to 9), with a primitive intermediate Unit 6. All samples in the upper group show slight enrichment in the light rare-earth elements (LREE; Fig. 15), with chondrite-normalized La/Sm ratios of around 1.6 (Table 1). Moderate positive Eu anomalies exist for Units 1 to 3 (Eu/Eu* ranges from 1.04 to 1.06; Table 1). The patterns for the normalized medium-to-heavy rare-earth elements (MREE to HREE) are essentially flat, with the more altered samples (794B-26R-2, 101-103 cm of Unit 1 and 794C-13R-1, 19-24 cm of Unit 5) showing MREE-HREE patterns that cross those of other samples. These crossing patterns likely represent HREE loss during alteration. The effects of alteration on Unit 5 chemistry are effectively shown by the "spider" plot of Figure 16, where increased large-ion lithophile elements (LILE) and decreased $\mathrm{Sr}, \mathrm{Y}$, and $\mathrm{Yb}$ contents with increased alteration are visible. This plot also illustrates that simple division of elements into elements that are "mobile" (left side of the spider plot) and "immobile" (right side of the spider plot) by aqueous fluids (e.g., Pearce et al., 1981) may not always be valid. Units 1,2 , and 5 show very similar patterns of relative enrichment and depletion (Fig. 16), with no sharp relative enrichments of any normalized element. However, Unit 3 contains substantially less relative $\mathrm{Nb}$ and $\mathrm{Ta}$, yielding significantly lower $(\mathrm{Nb} / \mathrm{Zr})_{\mathrm{N}}$ and $(\mathrm{Ta} / \mathrm{Hf})_{\mathrm{N}}$ (Table 1). As these pattern shapes and incompatible element ratios will not be significantly affected by fractional 

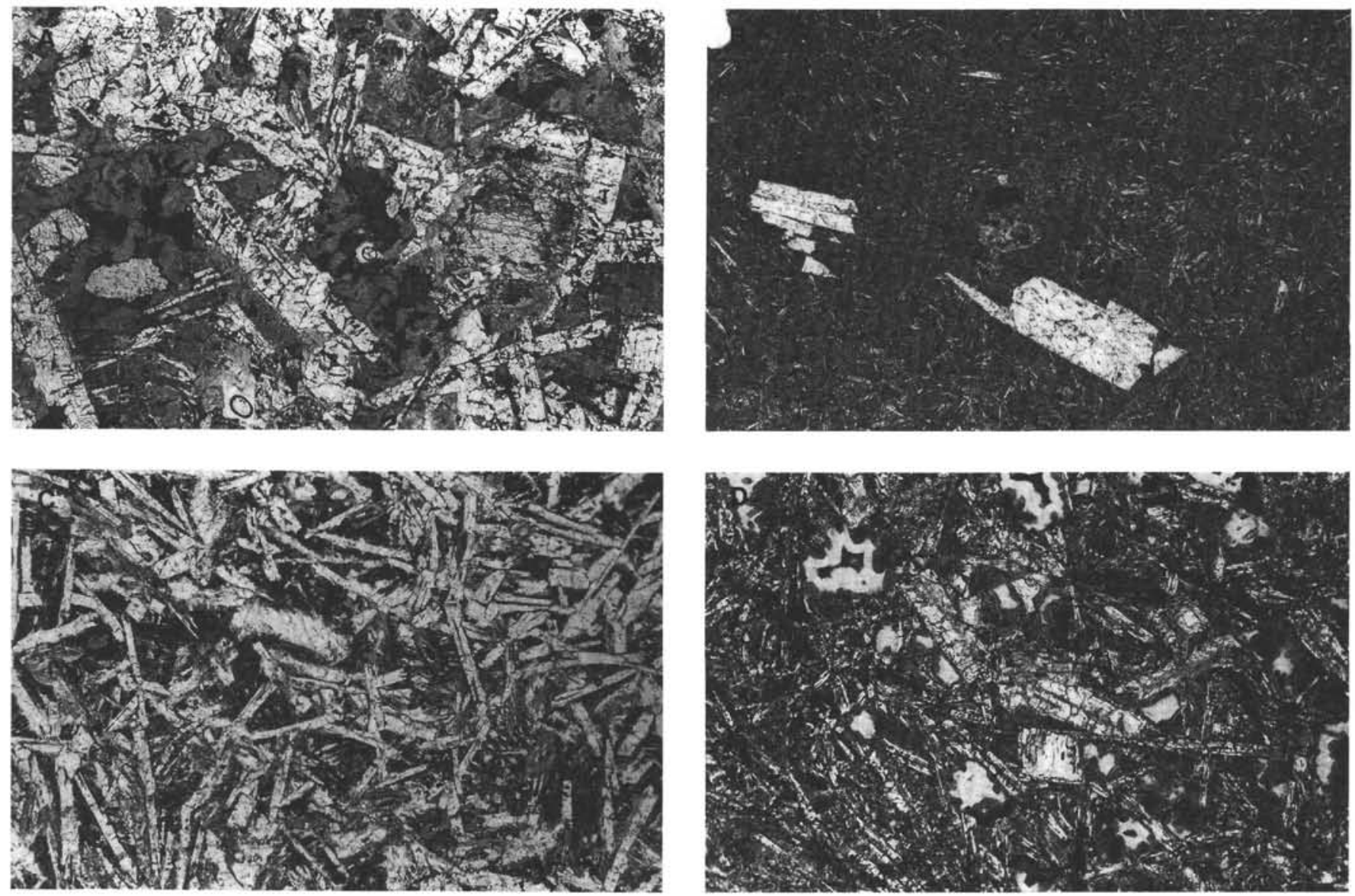

Figure 6. Photomicrographs of Unit 5, Site 794, and Unit 3, Site 797; each picture is $4 \mathrm{~mm}$ across. A. Coarse, doleritic interior of Unit 5 (127-794C-12R-4, 85-87 cm), with thick laths of plagioclase being incipiently altered to brown clays. Mesostasis and olivine are totally altered to mixed-layer brown clays, while ophitic and subophitic clinopyroxene is partially altered to these brown clays. Note the large fresh clinopyroxene in the center right of the photo; it is partially surrounded by alteration products. Anhedral magnetite is also visible. B. Totally altered quench margin of Unit 5 (Sample 127-794C-13R-1, 21-23 cm). The plagioclase phenocrysts have been replaced by adularia, while the olivine crystals in the center of the photograph have been replaced by dark greenish-brown mixed layer clays, as has the mesostasis. The Cr-spinel inclusions in the olivine have been replaced by pyrite. C. Coarse interior of Unit 3 (Sample 127-797C-12R-4,35-37 cm); the plumose and sheaf-radiating clinopyroxenes are partially replaced by secondary clays, as is the mesostasis. D. Highly altered margin of Unit 3 (Sample 127-797C-11R-1, 72-74 cm); the mesostasis is replaced by greenish-brown clays and the plagioclase is heavily altered to brown clays. Larger plagioclase crystals are replaced by adularia (more calcic originally and so more susceptible to low-temperature exchange?). Note that the vesicle infillings contain two generations of clays: an outer, lighter greenish-brown generation and an inner, dark-brown generation, probably reflecting variations in oxidation during different stages of alteration.

crystallization (Pearce, 1983; Wood et al., 1979; Erlank and Kable, 1976), several distinct magma batches must have been required.

As noted by Ingle, Suyehiro, von Breymann et al. (1990), most samples from Units 6 to 9 are strikingly different in composition to those stratigraphically above, with substantially lower amounts of all incompatible elements (Table 1; Figs. 15 and 16, where Sample 127-794D-13R$1,83-86 \mathrm{~cm}$, of Unit 7 represents the lower complex of Units 7 to 9). These units are characterized by relatively flat chondrite-normalized LREE to MREE ratios that cross those of the upper units (Fig. 15), with $(\mathrm{La} / \mathrm{Sm})_{\mathrm{N}}$ ranging from about 1.2 (Unit 6) to 0.8 (Unit 7). The MREE to HREE again show relatively flat patterns, with the exception of Unit 6 having a sharp, substantial positive Eu anomaly (Table 1). Overall, these units show a much smaller relative increase in the LILE relative to N-MORB than do Units 1 to 5 (Fig. 16), with Unit 7 giving a normalized pattern very similar to N-MORB with the exception of a slight relative depletion in Ta. Unit 6, in contrast, shows significant relative depletions in the HFSE $\mathrm{Zr}$ and $\mathrm{Hf}$, as well as low $\mathrm{Y}$ and $\mathrm{Yb}$. The relative lack of $\mathrm{Y}$ and $\mathrm{Yb}$ in this sample may partially reflect its high degree of alteration (LOI = 4.02; Table 1).

An exception to the above generalizations about Units 6 to 9 comes from Sample 127-794D-16R-1, 59-62 cm (Table 1). Our INAA data show that this sample is more enriched in the LREE than other samples from Units 6 to 9, and also more enriched than the other analyzed sample from Unit 8 (Table 1). Although this sample is highly altered (LOI of 4.57), it is doubtful that hydrothermal alteration could increase $\mathrm{La}, \mathrm{Ce}, \mathrm{Nd}$, and the relatively immobile element Ta simultaneously. Instead, it is possible that an additional unit exists, perhaps a thin dike difficult to map in core of poor recovery.

To produce the range in chemistry exhibited between the lower and upper sills, multiple parental lavas of differing composition derived from a somewhat heterogenous mantle source are required. Variations in partial melting of a single source cannot produce the strongly different N-MORB normalized elemental patterns seen in the "upper" and "lower" complexes (Pearce et al., 1981; Pearce, 1983), in particular the relatively depleted $\mathrm{Zr}$ and $\mathrm{Hf}$ of Unit 6. Nevertheless, the two complexes have similar $\mathrm{Sr}, \mathrm{Nd}$, and $\mathrm{Pb}$ isotopic compositions (Cousens and Allan, this volume; Nohda et al., this volume; Pouclet and Bellon, this volume). The presence of positive Eu anomalies in some samples (Eu/Eu*, Table 1) may be a possible result of plagioclase accumulation in the melt (Frey et al., 1974). The relatively high $\mathrm{Al}_{2} \mathrm{O}_{3}$ contents of these samples is consistent with this hypothesis. 

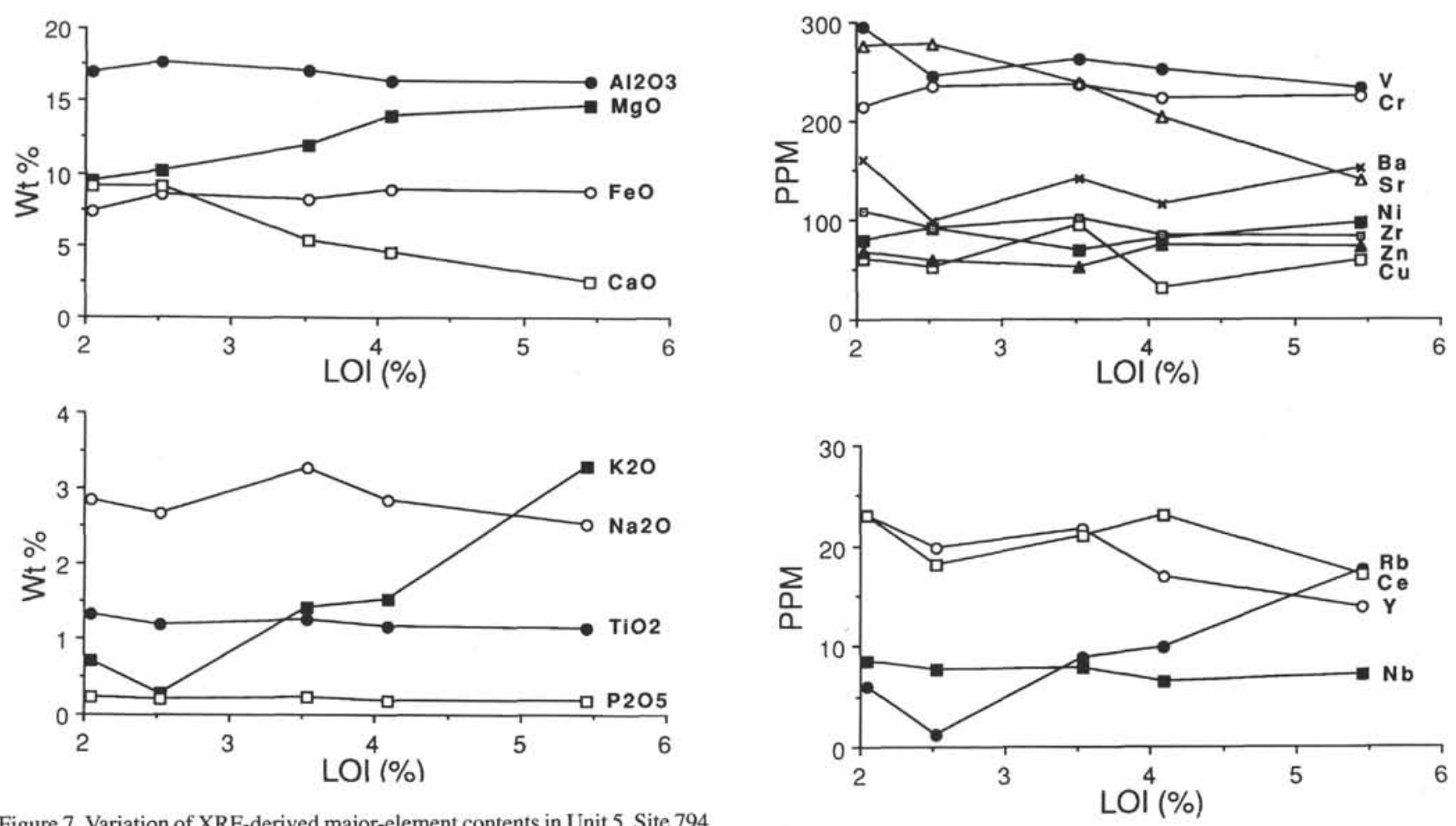

Figure 7. Variation of XRF-derived major-element contents in Unit 5, Site 794 with LOI (loss on ignition), a proxy for alteration. The most altered sample consists of $100 \%$ alteration minerals; higher LOI samples are located on the unit margins. Note opposite trends of $\mathrm{MgO}$ and $\mathrm{CaO}$, and strong correlation of $\mathrm{K}_{2} \mathrm{O}$ with LOI. Analytic precision ranges from $1 \%$ to $2.5 \%$ for the above elements and is $4 \%$ for LOI.

Taken as a group, the Site 794 samples are quite similar in chemistry and mineralogy to basaltic flows and doleritic sills of middle Miocene age found in the Akita-Yamagata oil fields and surrounding areas of coastal northwestern Honshu (Tsuchiya, 1988, 1989, 1990). These "oil field" basalts and dolerites were erupted and emplaced subaqueously during the initial opening of the Japan Sea (Tsuchiya, 1990), and show strikingly similar patterns of enrichments and depletions in N-MORBnormalized incompatible elements to the Site 794 rocks. Igneous rocks from both the oil fields and from Site 794 lack extensive $\mathrm{Nb}$ and $\mathrm{Ta}$ depletions; fresher samples exhibit smooth patterns of steady increases in normalized concentrations from $\mathrm{Yb}$ (less incompatible) to $\mathrm{Ba}$ (more incompatible). As noted by Tsuchiya (1990), these patterns are reminiscent of transitional-type MORB and less enriched oceanic island tholeiitic basalts (Sun et al., 1979; Pearce, 1983; Wilson, 1989), but are lower in incompatible elements and $(\mathrm{Sm} / \mathrm{Yb})_{\mathrm{N}}$ than most intraplate tholeitic lavas (e.g., Basaltic Volcanism Study Project, 1981; Menzies et al., 1984). Overall, they fall within the general range exhibited by backarc basin basalts (Saunders and Tarney, 1984; Pearce et al., 1981), lacking the strong "sawtooth" patterns caused by relative HFSE depletion and LILE enrichment of continental arc and many island arc basalts (Perfit et al., 1980; Pearce, 1982, 1983; Gill, 1981; Hickey et al., 1986; Arculus and Powell, 1986; Wood et al., 1979; Briqueu et al., 1984; Dupuy etal., 1982).

\section{Site 795, Northeastern Japan Basin}

Lavas from Site 795 fall into three basic eruptive groups (Fig. 4): an upper silicified and brecciated, relatively evolved moderately phyric clinopyroxene plagioclase basaltic andesite (Units 1 and 2); a massive, sparsely phyric clinopyroxene plagioclase basalt (Unit 3A), and a brecciated, sparsely phyric pyroxene plagioclase basalt to basaltic andesite (Unit 3B). All units are heavily altered, with the LOI ranging from $4.75 \%-5.99 \%, 2.84 \%-4.82 \%$, and $3.49 \%-5.88 \%$, re-

Figure 8. Variation of XRF-derived trace element contents in Unit 5, Site 794 with LOI. Note that $\mathrm{Sr}$ and $\mathrm{Rb}$ follow $\mathrm{CaO}$ and $\mathrm{K}_{2} \mathrm{O}$, respectively, in their behavior, and that $\mathrm{Y}$ declines with LOI. Analytical precision is $<3 \%$ for $\mathrm{V}, \mathrm{Cr}$, $\mathrm{Ni}, \mathrm{Sr}$, and $\mathrm{Zr}$, and $5 \%$ to $10 \%$ for $\mathrm{Zn}, \mathrm{Cu}, \mathrm{Ba}, \mathrm{Ce}, \mathrm{Y}, \mathrm{Rb}$, and $\mathrm{Nb}$.

spectively (Tamaki, Pisciotto, Allan, et al., 1990). Secondary green clays (celadonitic?) augmented by zeolites and cryptocrystalline silica fill the abundant vesicles, fractures, and voids between clasts, and secondary green clays replace the cores of most plagioclase phenocrysts. Unfortunately, the highly fractured and brecciated nature of the units precludes an analysis of element mobility similar to that conducted for Sites 794 and 797, although no consistent compositional trends with LOI were noted for the XRF data. Tamaki, Pisciotto, Allan, et al. (1990) found that the Site 795 lavas exhibited trends in $\mathrm{MgO}$ variation diagrams ( $\mathrm{Fe}$ and $\mathrm{V}$ depletion trends, and $\mathrm{Al}$ and $\mathrm{Si}$ enrichment trends) and a mineralogy (clinopyroxene and plagioclase phenocrysts; magnetite microphenocrysts in Unit 1) consistent with a calc-alkaline liquid line of descent. The samples are characterized by low amounts of the compatible elements $\mathrm{Ni}, \mathrm{Cr}$, and $\mathrm{MgO}$ (Table 1 ), indicating that they are all relatively evolved. In our discussion, we will concentrate on the fresher, more primitive samples of Units $1,3 \mathrm{~A}$ and $3 \mathrm{~B}$, with the chondrite-normalized REE plotted in Figure 17 and a range of N-MORB normalized incompatible elements plotted in Figure 18.

The REE contents of both Units $3 \mathrm{~A}$ and $3 \mathrm{~B}$ are very similar, exhibiting slight relative enrichment in the LREE and relatively flat patterns from Sm to Lu (Fig. 17). Slight Eu anomalies exist, with Eu/Eu* ranging from 1.04 to 1.06 . The "spider" plots of N-MORB normalized elements for Site 795 samples are also similar, with divergence between samples visible in the LILE (Fig. 18). These differences in the easily mobilized LILE could have resulted from variable alteration. The slight variability seen within the data could allow for both Units $3 \mathrm{~A}$ and $3 \mathrm{~B}$ to be part of the same eruptive event, with minor geochemical variation occurring during eruption. The normalized REE plot of Sample 127-795B-34R-1, 121-123 cm, from the more evolved Unit 1 is elevated and subparallel for most elements compared to those of Units $3 \mathrm{~A}$ and $3 \mathrm{~B}$. In contrast, a Eu anomaly is lacking (perhaps due to plagioclase fractionation), and a noticeable 

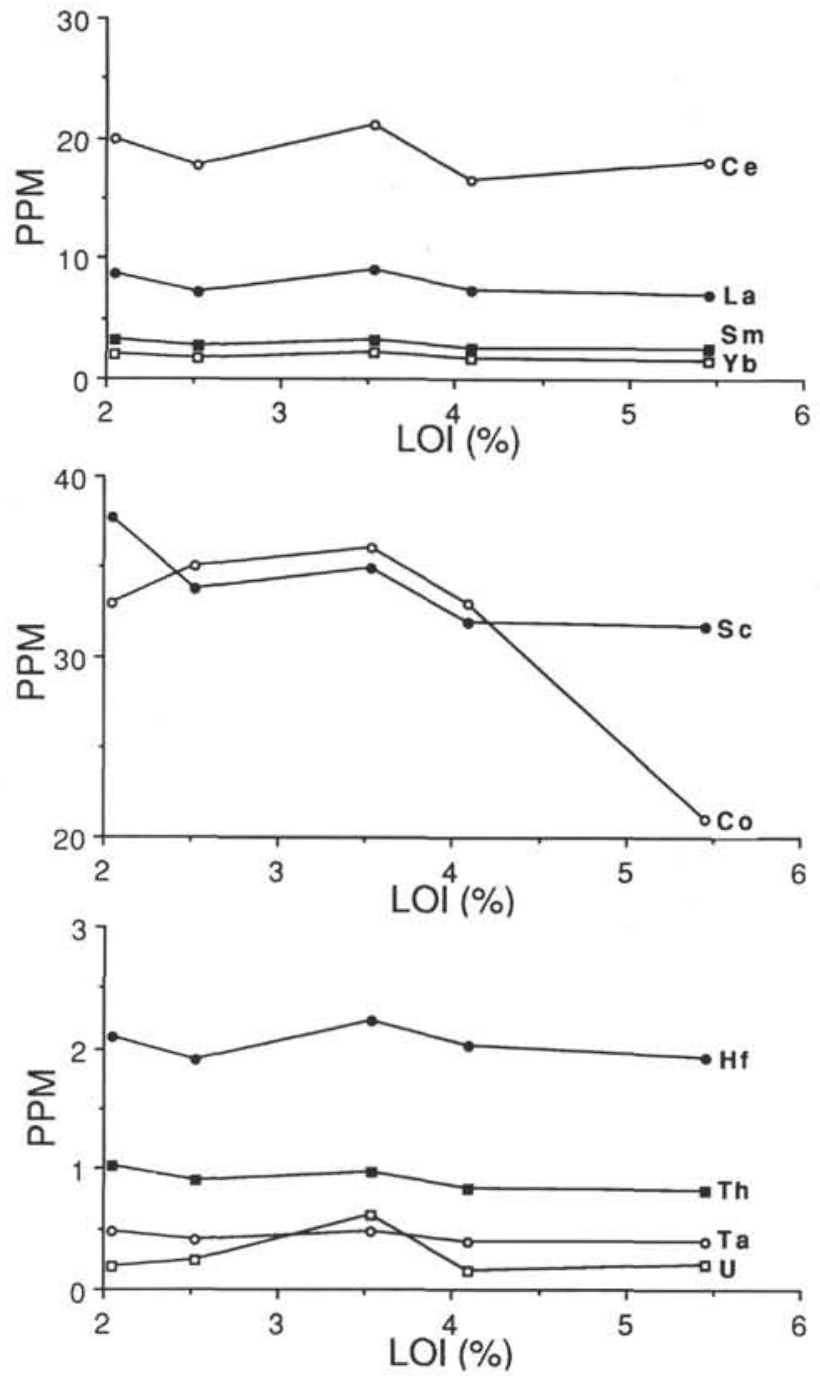

Figure 9. Variation of INAA-derived trace-element contents in Unit 5, Site 794 with LOI. Note that the REE in particular seem little affected by alteration, while Co strongly decreases in concentration with increasing LOI. Counting uncertainties for these elements are typically on the order of or smaller than the plotting symbols used ( $3 \%$ or less, except for $\mathrm{U}$ ).

dip in La as compared to Ce occurs. This unusual-looking dip could be due to $\mathrm{Ce}$ enrichment or La loss during alteration.

Figure 18 shows that the Unit 3A and 3B Site 795 lavas are lightly enriched in P, Ce, and the LILE compared to N-MORB, but are otherwise poor in incompatible elements. The depleted nature of these lavas is underscored by their relatively evolved character. Other key features include a moderate $\mathrm{Nb}$ and Ta depletion, and relatively low amounts of the HFSE Zr, Hf, and Ti. Unit 1 (Sample 127-795B-34R-1, $121-123 \mathrm{~cm}$ ) shows a different normalized element pattern, with less relative HFSE depletion. This difference in relative element enrichment and depletion rules out a parent-daughter genetic relationship between Unit 1 and Units $3 \mathrm{~A}$ and $3 \mathrm{~B}$. The samples lack the strong LILE enrichment and strong negative $\mathrm{Nb}$ and $\mathrm{Ta}$ anomalies typically associated with continental arc and more K-enriched island arc volcanics (e.g., Perfit et al., 1980; Pearce, 1982, 1983; Hickey et al., 1986; Arculus and Powell, 1986; Wood et al., 1979). Instead, they are more similar in composition to low-K, depleted oceanic island arc and backarc tholeiites and calc-alkaline basalts (Pearce et al., 1981; Saunders and Tarney, 1984; Wilson, 1989; Hawkins et al., 1990).
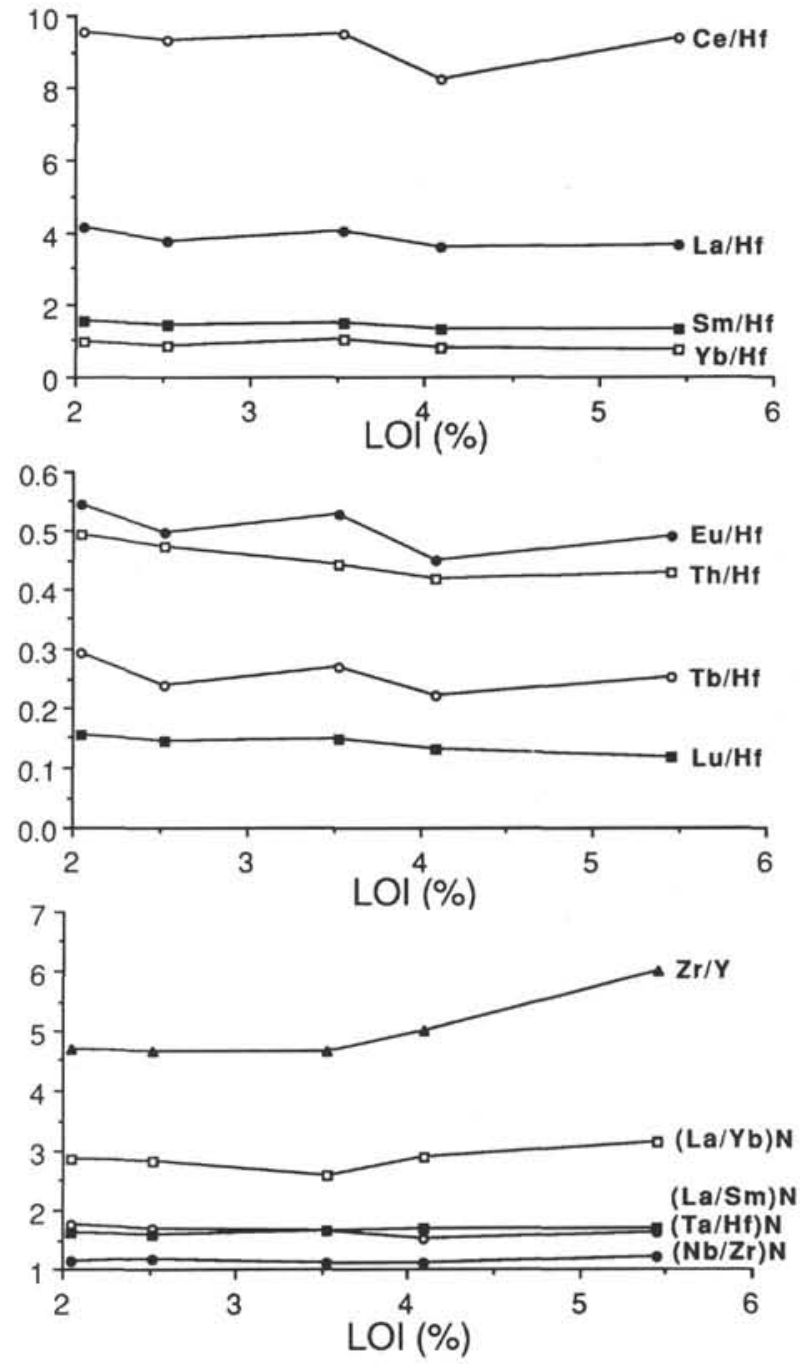

Figure 10. Site 794, Unit 5. The upper two graphs show REE and Th normalized by $\mathrm{Hf}$, assumed to be immobile (see text). This normalization removes the problem associated with rock volume changes during alteration, and allows a more quantitative approach toward elemental mobility. Note that the normalized elemental concentrations do not change with alteration except for minor declines by $\mathrm{Yb} / \mathrm{Hf}, \mathrm{Lu} / \mathrm{Hf}$, and $\mathrm{Th} / \mathrm{Hf}$ that represent a minor loss of the numerator elements. Errors in ratios from counting uncertainties are typically less than $1 \%$. The lower graph shows commonly used elemental ratios (from Table 1), with sharp increase in $\mathrm{Zr} / \mathrm{Y}$ representing the loss of $\mathrm{Y}$ in the more altered margins of the unit. The other elemental ratios show little change with LOI. Errors from counting uncertainties in these ratios are typically less than $1 \%$, except for $\mathrm{Zr} / \mathrm{Y}(4 \%)$ and $(\mathrm{Nb} / \mathrm{Zr})_{\mathrm{N}}(10 \%)$.

\section{Site 797, Western Yamato Basin}

Site 797 offered the deepest penetration ( $903 \mathrm{mbsf}$ ), the most units defined in core (21), and the most complex geochemistry of all the Japan Sea sites. Shipboard XRF analyses (Tamaki, Pisciotto, Allan, et al., 1990) together with our new INAA analyses are presented in Table 1 for Units 1 to 13, with INAA analyses for Units 15 and 21 also presented. As drilling proceeded until the very end of Leg 127, the shipboard party was unable to analyze all of the recovered units. $\mathrm{XRF}$ analyses from these lower units (Units 14 to 21 ) are presented in Table 2. Chondrite-normalized REE plots of the Site 797 units are plotted in Figure 19, and N-MORB normalized trace-element abundances are plotted in Figure 20. 

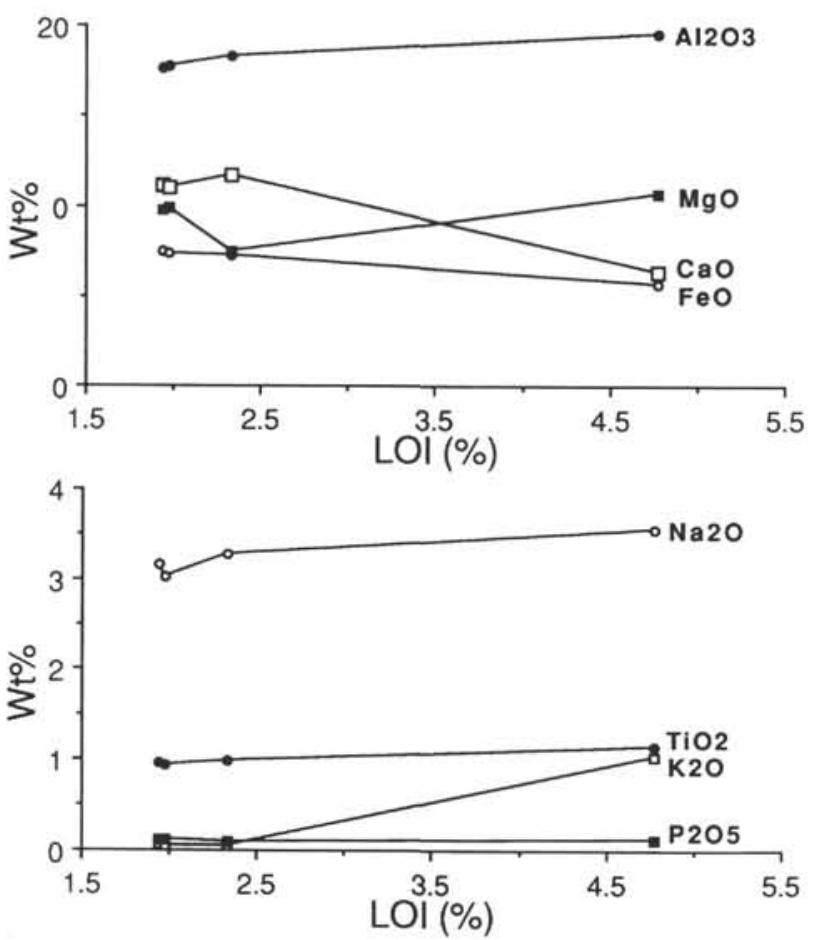

Figure 11. Variation of XRF-derived major-element contents in Unit 3, Site 797 with LOI (loss on ignition), a proxy for alteration. The most altered sample consists of nearly $100 \%$ alteration minerals; higher LOI samples are located nearer the unit margins. Note increasing $\mathrm{MgO}$ and $\mathrm{Al}_{2} \mathrm{O}_{3}$ and decreasing $\mathrm{CaO}$ and $\mathrm{FeO}$ trends with increasing LOI; these elements appear highly mobile with alteration. Note the lack of enrichment in $\mathrm{K}_{2} \mathrm{O}$; microprobe analysis of the unit margins show that $\mathrm{K}_{2} \mathrm{O}$ does indeed increase further toward the unit margins. Analytic precision ranges from $1 \%$ to $2.5 \%$ for the above elements and is $4 \%$ for LOI.

Tamaki, Pisciotto, Allan, et al. (1990) found two spatially-defined geochemical groups. The uppermost nine units were tentatively classified as high- $\mathrm{Al}$ basalts due to their lack of significant $\mathrm{Fe}$ enrichment and $\mathrm{Al}$ depletion trends with $\mathrm{MgO}$, and their similarity to other volcanic arc rocks in showing a relative depletion in $\mathrm{Nb}$. Nevertheless, the degree of alteration makes this interpretation questionable, and we note that the fresher samples resemble tholeiites. Some units appear to be quite primitive, with high $\mathrm{Ni}$ and $\mathrm{Cr}$ contents (Table 1). However, Allan (this volume) demonstrated that the very high $\mathrm{Mg} /\left(\mathrm{Mg}+\mathrm{Fe}^{2+}\right)$ of some upper group units is due to $\mathrm{MgO}$ enrichment during alteration. These basalts exhibit flat normalized REE patterns with slight relative depletions in the LREE and slight to moderate positive Eu anomalies (Fig. 19; Table 1). As with the Site 794 tholeiites, these anomalies suggest that the high rock $\mathrm{Al}_{2} \mathrm{O}_{3}$ could be associated with plagioclase accumulation in the melt rather than or as well as suppression of plagioclase crystallization by high magmatic water (Tamaki, Pisciotto, Allan, et al., 1990). The low $\mathrm{Yb}$ and Lu of Sample 127-797C-11R-1, 73-75 cm, is likely due to HREE loss during alteration, as mentioned earlier. As a group, these basalts are lower than typical N-MORBs in all of the incompatible elements except for Sr (Fig. 20). This curious lack of enrichment in the LILE is a feature of some backarc rocks from the actively spreading center of the Lau Basin (Hawkins and Melchior, 1985; Ernewein et al., in press). A principal difference between the upper group rocks of Site 797 and MORBs is their substantial relative depletion in the HFSE Ta and Nb (Fig. 20; Table 1).

Units 10 to 21 are strikingly different in composition from the overlying units, being more evolved and with substantially higher amounts of incompatible elements. Tamaki, Pisciotto, Allan, et al. (1990) classified the analyzed units on ship (Units 10-13) as enriched tholeiites and mildly alkaline basalts, as the samples plot within the
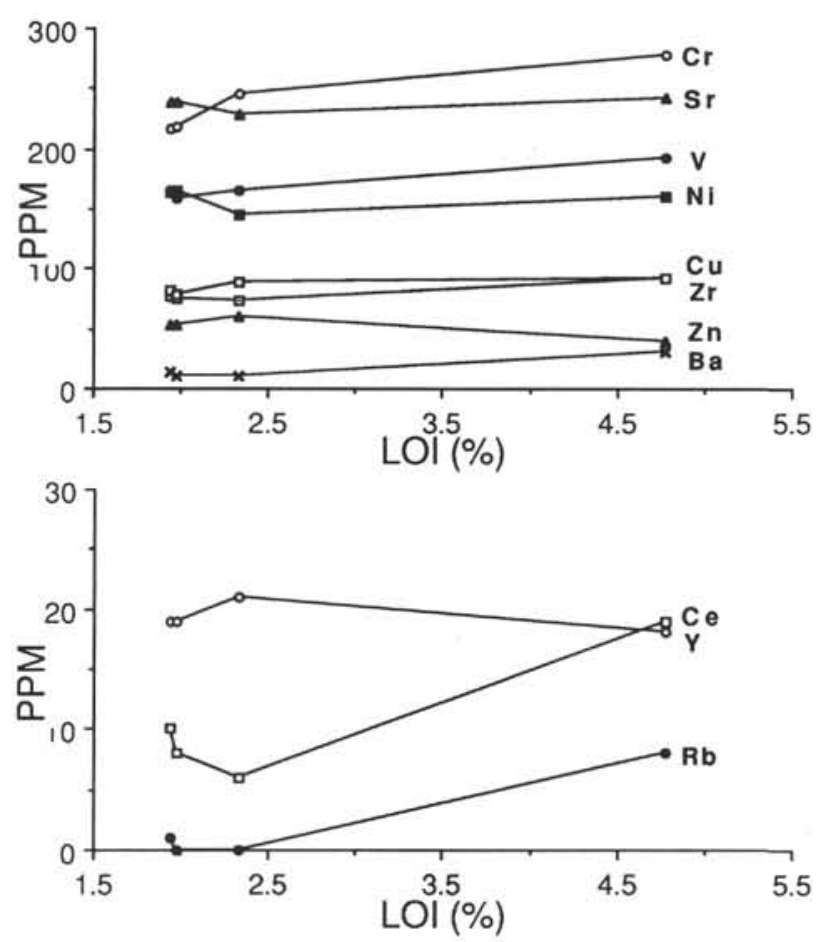

Figure 12. Variation of XRF-derived trace-element contents in Unit 3, Site 797 with LOI. Ce and $\mathrm{Rb}$ show strong increases with LOI, while $\mathrm{Y}$ again shows a decline with LOI. Analytical precision is $<3 \%$ for $\mathrm{V}, \mathrm{Cr}, \mathrm{Ni}, \mathrm{Sr}$, and $\mathrm{Zr}, 5 \%$ to $10 \%$ for $\mathrm{Zn}, \mathrm{Cu}, \mathrm{Y}$, and $\mathrm{Rb}$, and $20 \%$ for $\mathrm{Ce}$ and $\mathrm{Ba}$.

alkaline field of Kuno but lack normative nepheline. The degree of alteration makes classifications such as these difficult. The experiments of Thy (this volume) show that the major-element compositions of these units could be explained by low-pressure fractionation of tholeiitic parental liquids, roughly similar in composition to those of the upper group (but more enriched in the incompatible elements). As a result, we refer to these lower units as enriched tholeiites. Thinsection and XRF analyses of the lowermost units (15 to 21) show them to be highly altered (note the low $\mathrm{CaO}$ and high LOI contents of some in Table 2), with a range in composition lower in incompatible elements than Units 10-13 but higher than Units 1 to 9 . Their degree of alteration makes analysis of them problematic, so we will limit our discussion of these to Units 15 and 21 .

Units 10 to 15 exhibit parallel chondrite-normalized REE patterns, with moderately high LREE enrichments (chondrite-normalized La/Sm averages around 1.6) and with slight negative slopes in the medium to heavy REE (Fig. 19). All have slightly negative Eu/Eu*, consistent with therelatively low $\mathrm{Al}_{2} \mathrm{O}_{3}$ and $\mathrm{CaO}$ and moderate $\mathrm{Sr}$ (in the fresher samples) that imply derivation from liquids that have undergone substantial plagioclase fractionation (Frey et al., 1974). The high concentration of V in these samples is consistent with a tholeiitic liquid line of descent that has undergone little or no magnetite fractionation, due to the strong partitioning of this element into magnetite (Irving, 1978). Unit 21 has less LREE enrichment, producing a pattern that crosses the others (Fig. 19). These REE patterns show that parental lavas that produced the lower suite of units at this site were substantially more enriched in the LREE than those that produced Units 1 to 9. Variation in LREE enrichment was also present in the parental lavas of the lower suite, implying several eruptive episodes.

Figure 20 shows that this stratigraphically lower group of units is substantially enriched in most of the LILE elements with respect to $\mathrm{N}$-MORB, excepting Sr. Units 10 and 12 are the most enriched of the group. Most high-field strength elements are also enriched, yet Ta and $\mathrm{Nb}$ show mild relative depletions when compared to the other incompatible elements. Nevertheless, these concentrations of $\mathrm{Ta}$ and $\mathrm{Nb}$ are 

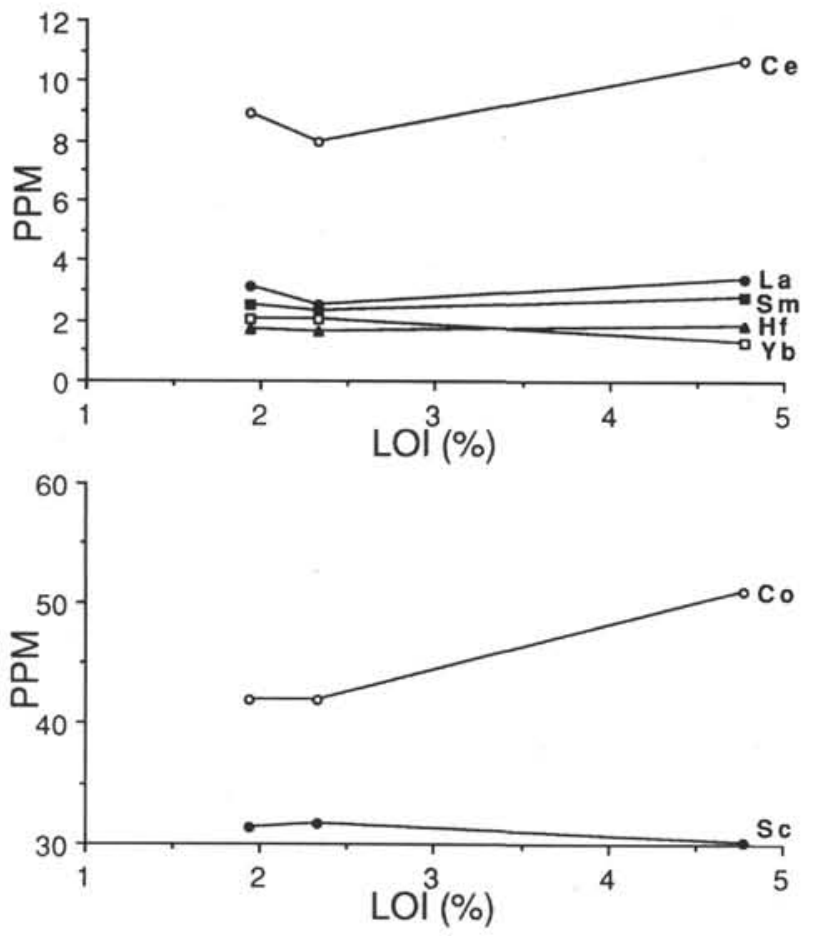

Figure 13. Variation of INAA-derived trace-element contents in Unit 3, Site 797 with LOI. Yb notably declines with LOI (as does Lu; see Table 1), while Ce strongly increases with LOI. Opposite to Unit 5 of Site 794, Co strongly increases in concentration with increasing LOI. Counting uncertainties for these elements are typically on the order of or smaller than the plotting symbols used ( $3 \%$ or less, except for $\mathrm{U})$.

still higher than in N-MORB. These samples contain substantially more $\mathrm{Ta}, \mathrm{Nb}$, and $\mathrm{Ti}$ and substantially less $\mathrm{Sr}$ than typical arc basalts, and are more enriched in the incompatible elements (particularly $\mathrm{Ta}$, $\mathrm{Nb}$, and $\mathrm{Ti}$ ) than most backarc or marginal basin basalts (e.g., Saunders and Tarney, 1984; Pearce et al., 1981; Pearce, 1982, 1983; Wilson, 1989). They fall within the range of the Akita-Yamagata "oil field" basalts (Tsuchiya, 1990), and compare with oceanic island tholeiitic compositions, continental flood tholeiites, and T-MORB compositions (Pearce et al., 1982; Wilson, 1989, Basaltic Volcanism Study Project, 1981; Menzies et al., 1984; Wood et al., 1979; Michael et al., 1989), differing only in their somewhat lower $\mathrm{Ta}$ and $\mathrm{Nb}$ contents, which may indicate a slight subduction zone signature. Overall, they are quite similar in their relative pattern of enrichment to DSDP samples from the Guaymas Basin (Saunders et al., 1982). These N-MORB normalized element patterns, so different in shape from those of the upper group of units, require that the mantle sources of the lower units were more enriched in incompatible elements than those of the upper nine units (Pearce et al., 1981; Pearce, 1983). This conclusion is consistent with the isotopic results of Cousens and Allan (this volume), who show that the lower group of units has more radiogenic $\mathrm{Sr}$ and $\mathrm{Pb}$ and less radiogenic $\mathrm{Nd}$ isotopic compositions than those of the upper group. They argue that isotopic and mass-balance constraints indicate that these differences could not have resulted from continental crustal assimilation, an argument that is supported by the low Sr of Units 10 to 21 (Table 1).

\section{DISCUSSION}

A major goal of Legs 127 and 128 was to understand the nature and composition of the backarc crust (Tamaki, Pisciotto, Allan, et al.,
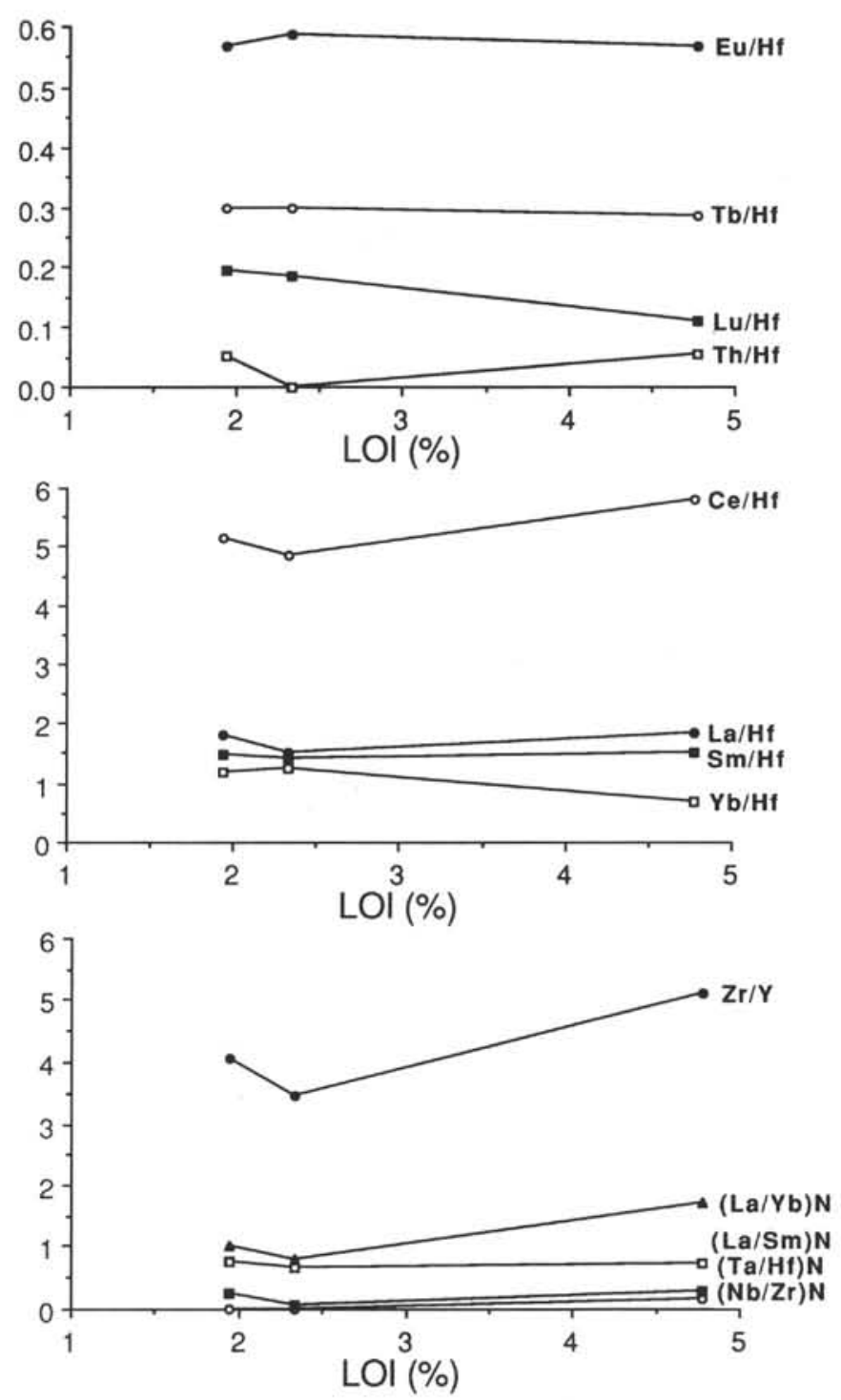

Figure 14. Site 797, Unit 3. The upper two graphs show REE and Th normalized by Hf, assumed to be immobile (see text). This normalization removes the problem associated with rock volume changes during alteration, and allows a more quantitative approach toward elemental mobility. The normalized elemental concentrations $\mathrm{Yb} / \mathrm{Hf}$ and $\mathrm{Lu} / \mathrm{Hf}$ illustrate the relative loss of the HREE during alteration. Errors in ratios from counting uncertainties are typically less than $1 \%$, with the exception of $\mathrm{Th} / \mathrm{Hf}(20 \%$, due to low values of Th). The lower graph again shows commonly used elemental ratios (from Table 1), with the sharp increase in $\mathrm{Zr} / \mathrm{Y}$ and $(\mathrm{La} / \mathrm{Yb})_{N}$ representing the relative loss of the geochemically similar $\mathrm{Y}$ and HREE in the more altered margins of the unit. The other elemental ratios again show little change with LOI. Errors from counting uncertainties in these ratios are typically less than $1 \%$, except for $(\mathrm{Ta} / \mathrm{Hf})_{\mathrm{N}}(15 \%)$ and $(\mathrm{Nb} / \mathrm{Zr})_{\mathrm{N}}(50 \%)$ due to very low $\mathrm{Ta}$ and $\mathrm{Nb}$.

1990; Ingle, Sueyihiro, von Breymann et al., 1990). These data show that the composition of igneous rocks in the uppermost crust of the Japan Sea are broadly similar to those found in other marginal basins, in that they fall between traditional classifications of arc tholeiites, MORBs, intraplate basalts, and calc-alkaline basalts, sharing various signatures of these "end-member" compositions (Volpe et al., 1987). This explains our rationale for using "spidergrams" that illustrate 

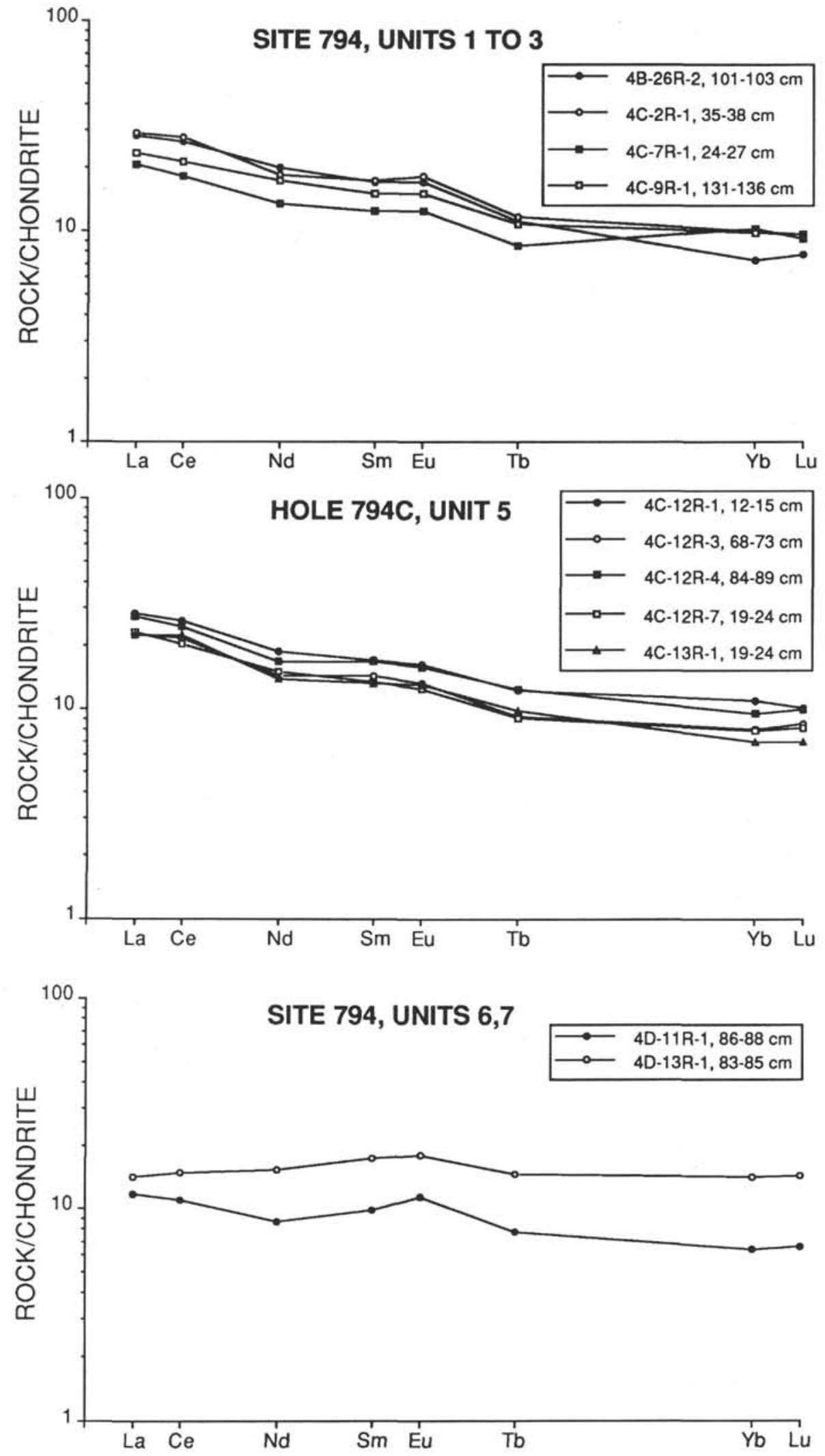

Figure 15. Chondrite-normalized (Masuda et al., 1973) REE contents of Site 794 tholeiites. Note the crossing patterns of the stratigraphically lower samples (Samples $127-794 \mathrm{D}-11 \mathrm{R}-1,86-88 \mathrm{~cm}$, and $-13 \mathrm{R}-1,83-86 \mathrm{~cm}$, from Units 6 and 7) with those from the upper group of units (Units 1 to 5). The pattern in the medium to heavy REE of Sample 127-794C-13R-1, 19-24 cm, slightly crosses those of the other less-altered Unit 5 samples, indicating relative heavy REE loss. 

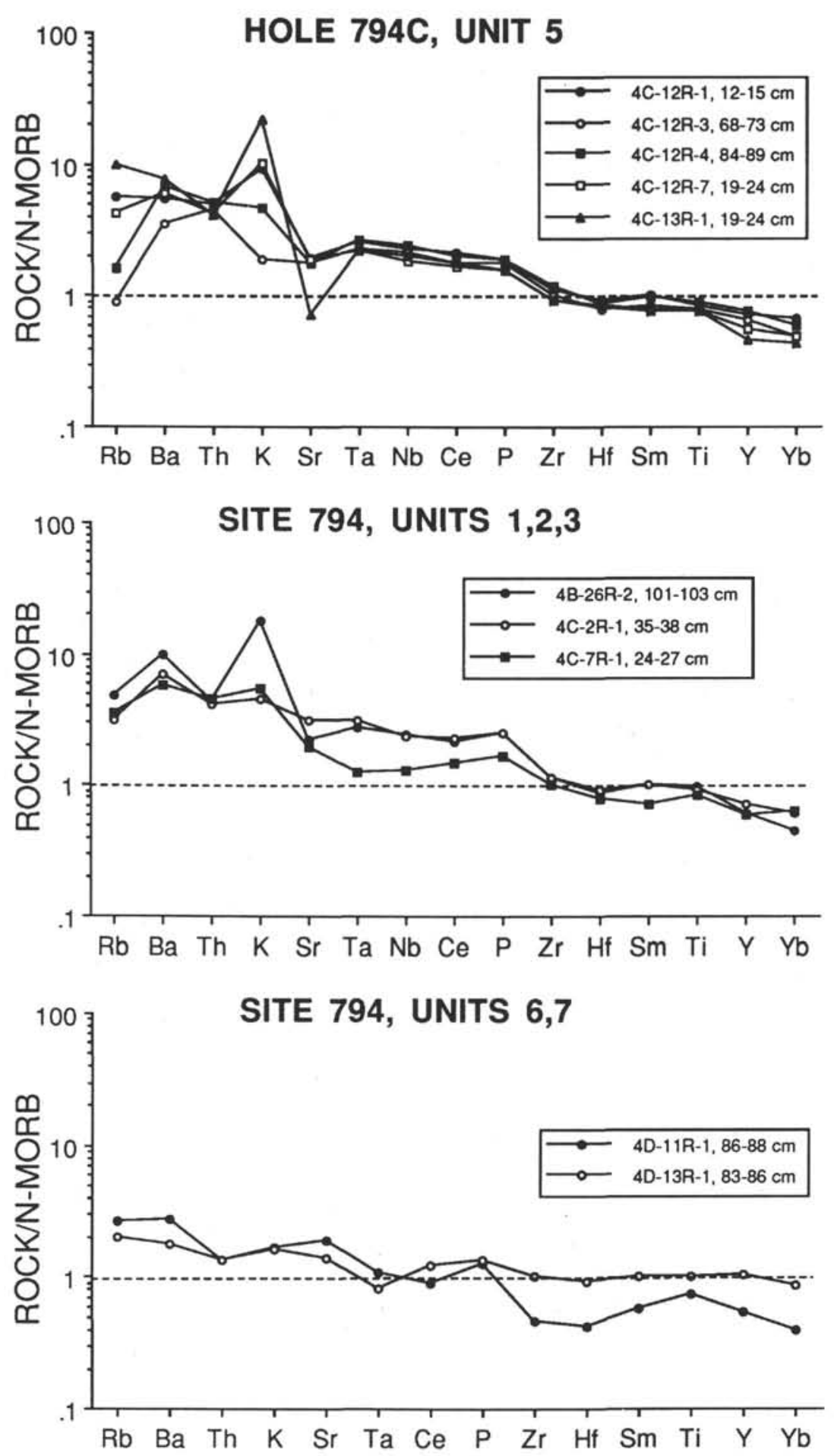

Figure 16. "Spidergrams" of N-MORB normalized element plots of Site 794 samples, using normalizing values from Pearce et al. (1981) with slight modification in the order of the elements. Data plotted in the graphs is from the INAA data when multiple analyses of elements occurred, to minimize problems of mixing data derived from slightly offset portions of core in the XRF and INAA analyses. The effect of alteration on the large-ion lithophile elements (LILE) is graphically shown by the plotted Unit 5 samples. The stratigraphically lower samples (Samples 127-794D-11R-1, 86-88 cm, and-13R-1, 83-86 $\mathrm{cm}$, from Units 6 and 7, respectively) are much less enriched in these incompatible elements. None of the samples contain strong relative $\mathrm{Nb}$ and $\mathrm{Ta}$ depletions and $\mathrm{Sr}$ enrichments, features traditionally associated with a subduction-related signature (see text for discussion). 


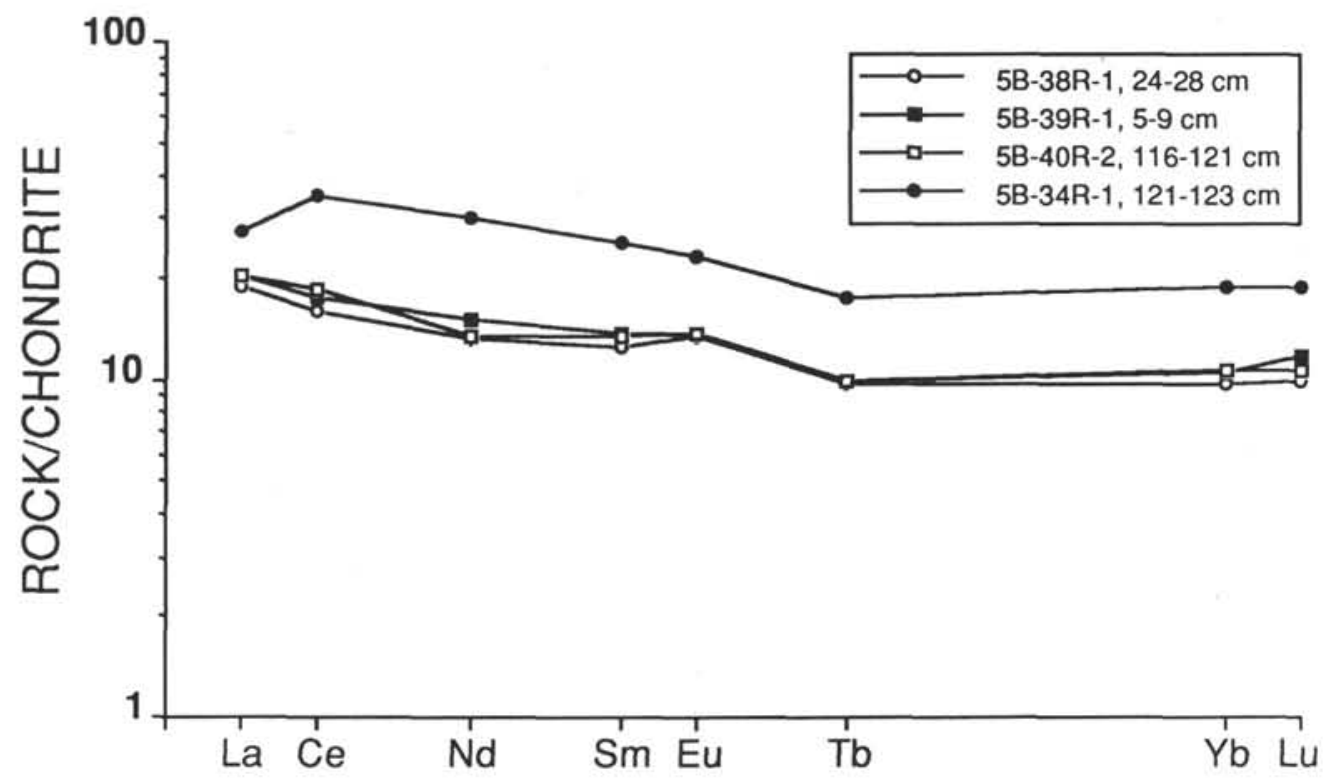

Figure 17. Chondrite-normalized (Masuda et al., 1973) REE contents of Site 795 basaltic andesites and andesite. The samples from Units $3 \mathrm{~A}$ and $3 \mathrm{~B}$ are quite similar, consistent with representing a single eruption. In contrast, the overlying Unit 1 (Sample 127-795B-34R-1, 121-123 cm) has more than twice the Sm, Nd, and Ce and a different pattern of the normalized REE, indicating that it must represent a different eruptive event.

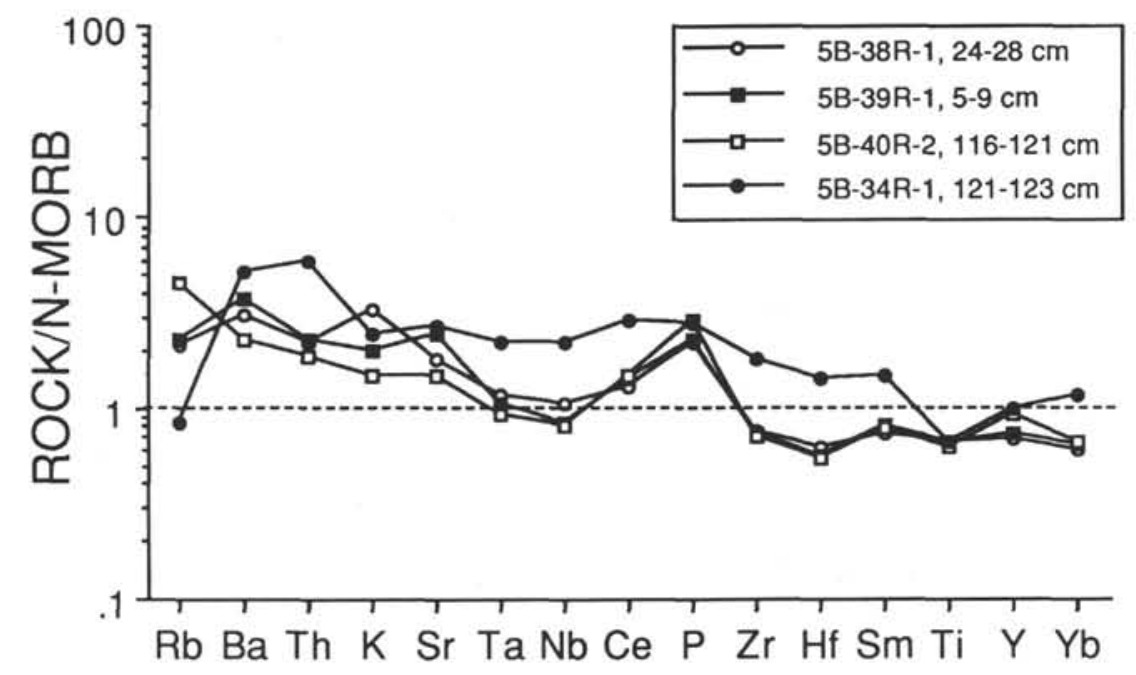

Figure 18. "Spidergrams" of N-MORB normalized element plots of Site 795 samples, using normalizing values from Pearce et al. (1981) with slight modification in the order of the elements. Data plotted in the graphs is from the INAA data when multiple analyses of elements occurred, to minimize problems of mixing data derived from slightly offset portions of core in the XRF and INAA analyses. Sample 127-795B-34R-1, 121-123 cm, of Unit 1 has a substantially different pattern than those of Units $3 \mathrm{~A}$ and $3 \mathrm{~B}$, indicating genesis from a different parent and also from a source relatively more enriched in the high-field strength elements (HFSE) $\mathrm{Ta}, \mathrm{Nb}, \mathrm{Zr}$, and Hf. Overall, the Site 795 samples are depleted in the incompatible elements, and lack strong depletions in the HFSE and LILE, inconsistent with eruption within the framework of a continental volcanic arc (see text).

numerous elements instead of just simple elemental ratios for data analysis. Despite their complexity, these samples yield important constraints on the nature of the crust and underlying mantle of the Japan Sea.

A goal at Site 795 of Leg 127 was to understand the nature and history of the crust of the northern Japan Basin margin. The crustal thickness of this portion of the Japan Basin is slightly greater than
11-12 km, and so thicker than typical oceanic crust (7-10 km; Ludwig et al., 1975; Tamaki, Pisciotto, Allan, et al., 1990). Our data are consistent with the interpretation of the Leg 127 shipboard party that the Site 795 basement rocks could represent subaqueous lavas erupted within the framework of a rifting oceanic arc, perhaps at an early stage of back-arc basin formation. The lack of LILE enrichment and the overall highly depleted nature of the samples argue strongly against 

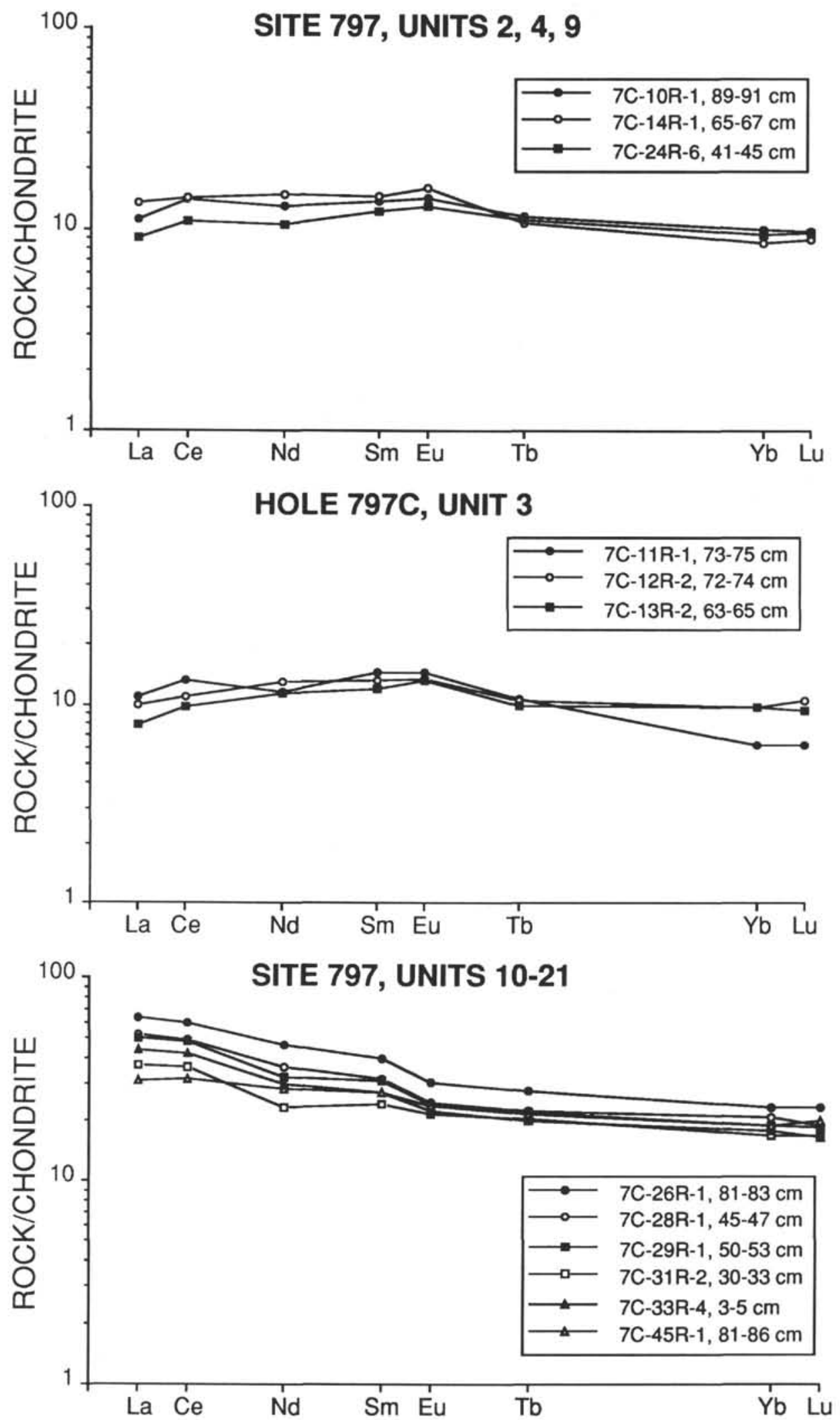

Figure 19. Chondrite-normalized (Masuda et al., 1973) REE contents of Site 797 tholeiites. Note the depleted nature of the upper group of Units 1 to 9 in the LREE, and the enriched nature of the stratigraphically lower Units 10 to 21. Note also the depletion of the heavy REE in Sample 127-797C-13R-2, 63-65 cm (Unit 3), most likely due to loss during alteration. 

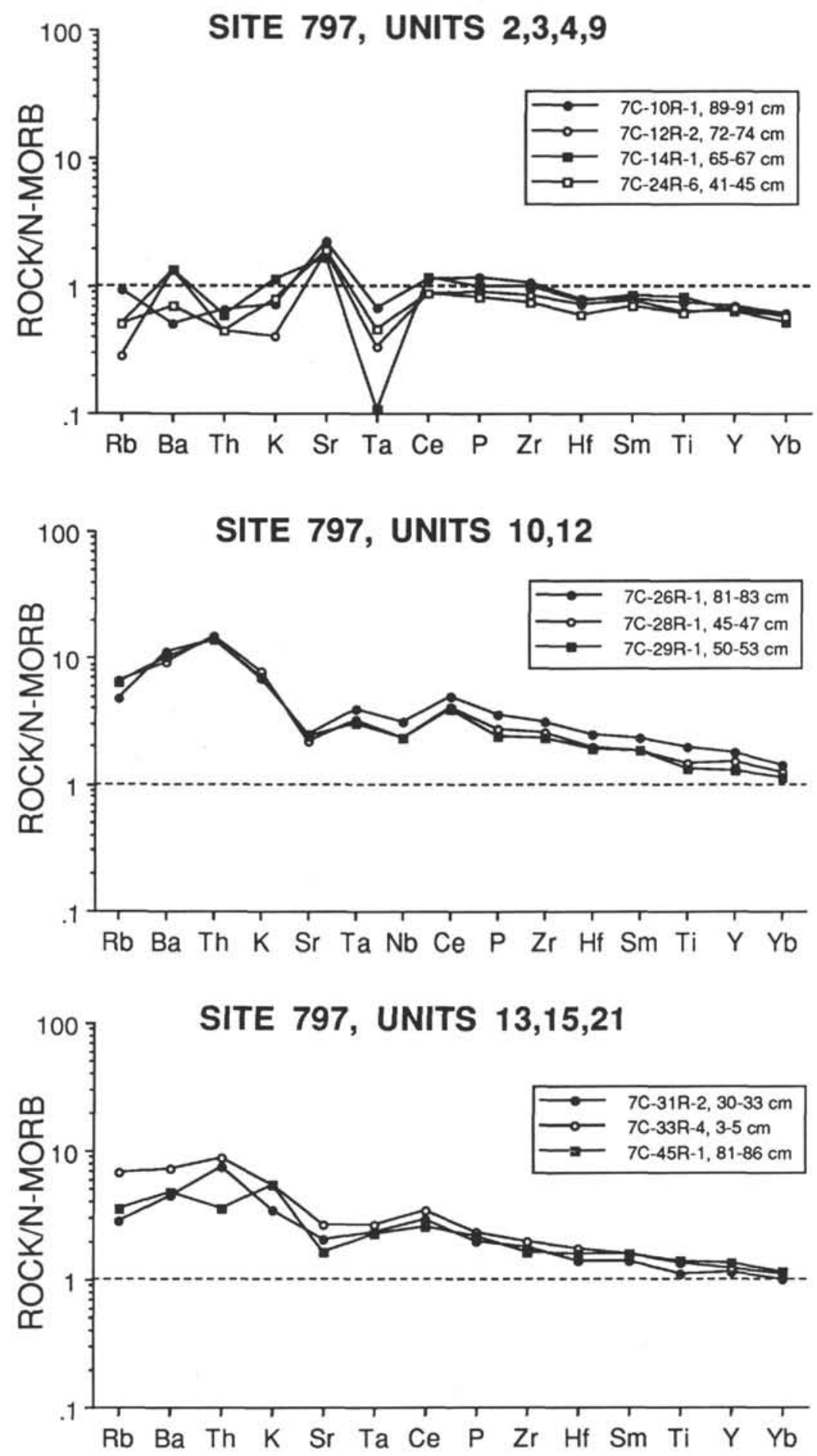

Figure 20. "Spidergrams" of N-MORB normalized element plots of Site 797 samples, using normalizing values from Pearce et al. (1981) with slight modification in the order of the elements. Data plotted in the graphs is from the INAA data when multiple analyses of elements occurred, to minimize problems of mixing data derived from slightly offset portions of core in the XRF and INAA analyses. Note the depleted character of the upper nine units and their strong relative depletion in the HFSE Ta. In contrast, the lowermost 12 units are enriched in all of the incompatible elements, the LILE (except for $\mathrm{Sr}$ ) in particular. 
the involvement of continental crust or subcontinental mantle in their genesis, indicating that the pre-Japan Sea arc composition in the region was oceanic, not continental, in nature.

Sites 794 (on the central northern basin margin) and 797 (on the western basin margin) were designed to investigate the crustal nature and origin of the Yamato Basin, an enigmatic portion of the Japan Sea in terms of its anomalously thick crustal thickness (17-19 km; Ludwig et al., 1975; Hirata et al., 1989; Tamaki, Pisciotto, Allan, et al., 1990). The majority of units sampled represent sills or dikes, intruded into soft sediment during rapid deepening associated with extension (Tamaki, Pisciotto, Allan, et al., 1990). The fact that depth-defined geochemical groups of sills and lavas occur implies that rock chemistry varies with age at both sites; yet curiously, the lower group of sills are the more enriched in incompatible elements at Site 797, while the lower group of sills at Site 794 are the more depleted in these elements. If the sills at both sites intruded soft sediment as envisioned by Einsele (1982) at the Guaymas Basin, then the stratigraphically higher units at each site were younger, intruding earlier sills by fracture-propagation before reaching the less-dense, nonfracturable, soft sediment. Unfortunately, the dates reported by Kaneoka et al. (this volume) and Pouclet and Bellon (this volume) for differing units at Site 794 cannot solve this problem, as the dates for upper and lower units at each site are not resolvable at their levels of reported error. At Site 797, the lower and upper sill groups intruded similar laminated sandstones, siltstones, and claystones that were rapidly deposited (Tamaki, Pisciotto, Allan, et al., 1990), implying that the time difference between the intrusion of the upper and lower group of sills was small. As none of the dated samples were treated to remove alteration, the Yamato Basin ${ }^{40} \mathrm{Ar} /{ }^{39} \mathrm{Ar}$ plateau ages (Kaneoka et al., this volume) yield the data most likely approximating the age of intrusion. It is a crucial point that these ages, ranging from 17.7-21.2 $\mathrm{Ma}$ for both sites, are not resolvable within analytic error to a $95 \%$ confidence level. This indicates that the age range of the sampled units in the Yamato Basin is small and that basin extension was widespread by about $18-21 \mathrm{Ma}$.

Our data show that the igneous rocks collected by coring in the Yamato Basin are a diverse group, with strong compositional variation between and within the sites that indicates the mantle underlying the Yamato Basin is compositionally heterogenous. Considering the Leg 127/128 sample set as a whole, our geochemical data show a good correlation of LREE enrichment with more radiogenic $\mathrm{Sr}$ and $\mathrm{Pb}$ and less radiogenic $\mathrm{Nd}$ isotopic composition (Cousens and Allan, this volume). The isotopic data define what appears to be two- component mixing between a depleted MORB-type (DM) source and a relatively radiogenic (EM) source. The mixing line is best developed in the $\mathrm{Pb}$ systematics, where correlation coefficients are quite high (e.g., 0.997 for ${ }^{207} \mathrm{~Pb} /{ }^{204} \mathrm{~Pb}$ vs. ${ }^{206} \mathrm{~Pb} /{ }^{204} \mathrm{~Pb}$ and 0.999 for ${ }^{208} \mathrm{~Pb} /{ }^{204} \mathrm{~Pb}$ vs. ${ }^{206} \mathrm{~Pb} /{ }^{204} \mathrm{~Pb}$ at Site 797). Simple mixing calculations suggest that $0.5 \%$ to $2.5 \%$ of the $\mathrm{Pb}$ isotopic signature is due to a radiogenic EM2 (Zindler and Hart, 1986) contaminant deduced to be oceanic sediment incorporated in the source by subduction processes (Cousens and Allan, this volume). The contaminant was probably not continental crust nor old sub-continental lithosphere, because (1) there is no correlation between $\mathrm{Mg}$ number (magmatic evolution) and $\mathrm{Pb}$ or $\mathrm{Sr}$; (2) for Japanese crustal rocks, published ${ }^{87} \mathrm{Sr} /{ }^{86} \mathrm{Sr}$ ratios are too high and ${ }^{143} \mathrm{Nd} /{ }^{144} \mathrm{Nd}$ ratios far too low for them to be considered as a contaminant; and (3) inferred lithospheric and sublithospheric compositions derived from eastern Chinese basalts are instead consistent with an EM1 (Zindler and Hart, 1986)-type contaminant. The relatively low amount of $\mathrm{Sr}$ and $\mathrm{K}_{2} \mathrm{O}$ in the fresher Yamato Basin samples are consistent with the hypothesis that these samples have interacted little with continental crust. Thus, the isotopic data strongly suggest a subduction zone signature, with a substantial portion of the mantle heterogeneity derived from elemental fluxes associated with subducted material.

Nevertheless, our data suggest that the mantle heterogeneity is comprised of at least three components, with the subduction-related elemental flux superimposed on pre-existing mantle heterogeneity. Figure 21 shows a plot of elemental ratios that has been put forward as a way of identifying relative crustal and mantle source contributions (Pearce, 1983). The

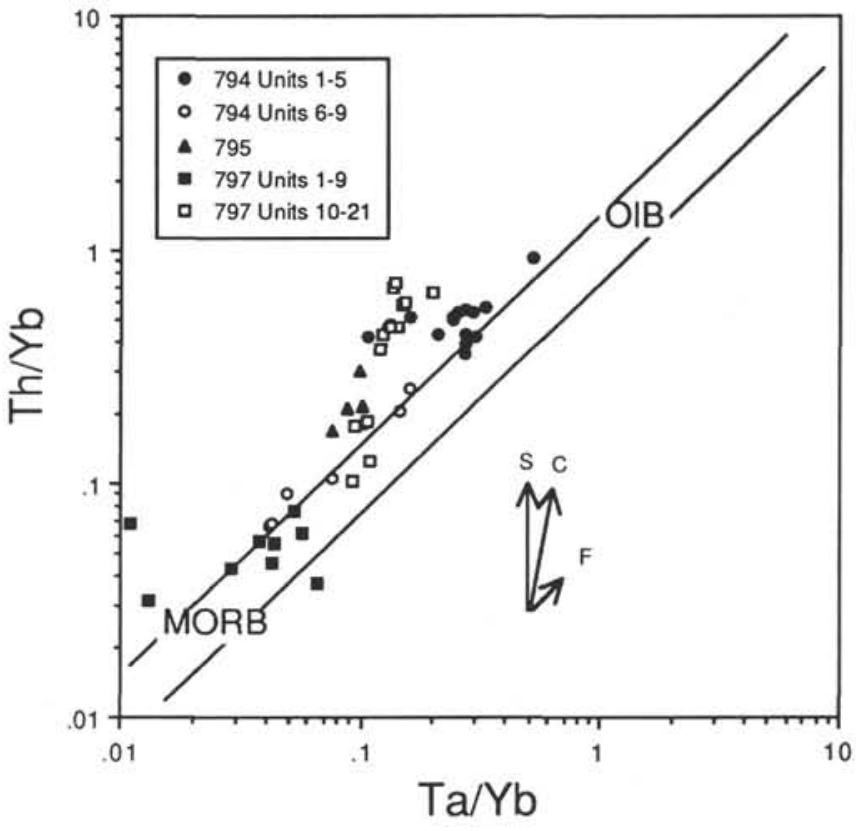

Figure 21. Plot after Pearce (1983) illustrating various possible contributions to magma chemistry in $\mathrm{Th} / \mathrm{Yb}$ vs. $\mathrm{Ta} / \mathrm{Yb}$ space. Mantle mixing array between depleted MORB source depleted mantle and ocean island basalt source enriched mantle (OIB) is shown, as are arrows illustrating how assimilation of subduction components (sediment) into the mantle source ( $\mathrm{S}$ ) and assimilation of continental arc crustal material into the rising magma (C) affects the composition of magma in an arc or back-arc system. Effect of partial melting (F) shown by the small arrow; its effect is small over the range of partial melting of interest (likely several to about $20 \%$ ) and nearly parallel to the mixing line (Th being slightly more incompatible than $\mathrm{Ta}$, when no residual titanates are present in the mantle residues). Crystal fractionation in the parental magmas of the Leg 127 and 128 rocks will change these compositional ratios little. The slope of the array defined by the Leg 127/128 rocks, especially those of Site 797 , is greater than that defined by the MORB-OIB mixing array but less than that defined by assimilation of crust or source enrichment by subduction components. The width of the array is also substantially greater than analytic precision (the size of the plotting symbols when $\mathrm{Ta} / \mathrm{Yb}$ and $\mathrm{Th} / \mathrm{Yb}$ are $>0.1$ ). The slope and breadth of the array reflects the need for a minimum of three components in the mantle source.

relative contributions of different contaminants and source contributions are shown by the arrows (see figure caption for explanation). As with the isotopes, the data broadly define a linear array, with this array offset to a higher slope from the hypothesized oceanic island basalt (OIB)-MORB source mixing array. The defined slope is shallower than that likely to result from crustal assimilation (unlikely on isotopic grounds) or from mixing subduction components into the source mantle, and the data array is much broader than analytical error allows for simple two-component mixing. Therefore, at least three mantle sources are necessary to explain the data (not necessarily an OIB-type source per se). This conclusion is supported by the fact that the isotopic data on Site 794 samples do not differentiate between the upper and lower groups of different units at the site, despite their very different compositional characteristics.

A key feature of all of Leg 127 and 128 samples is their flat medium-to-heavy chondrite-normalized REE patterns; this argues strongly that the primary magmas producing the samples in both the Yamato and Japan Basins last equilibrated with a shallow garnet-free mantle of spinel (and perhaps plagioclase-bearing) lherzolite composition (Hansen, 1989; Frey et al., 1978; Basaltic Volcanism Study Project, 1981; Schilling, 1975), at pressures of 10-25 kbar (Green et al., 1979; Basaltic Volcanism Study Project, 1981; Takahashi and 
Kushiro, 1983). The high-pressure experimental data of Yamashita et al (this volume) is consistent with this inference; they estimated that the primary melts for the upper Site 797 units were generated at about $14 \mathrm{kbar}$ from spinel lherzolite. Therefore, the primary melts for the Leg 127/128 samples were derived from a heterogenous and shallow mantle source, where melt segregation took place at depths of around $30-40 \mathrm{kbar}$ with little subsequent interaction with the overlying and extending lithosphere. The age range of the sampled units indicates that the mantle source must have been heterogenous on both a broad scale (over the range of the Yamato Basin) and on a local, site-specific scale (such as Site 797) over a few Ma range in time. These data require that the source heterogeneity consists of an intimate mixture of relatively enriched and depleted mantle (plum-pudding model). Therefore, the data do not lend support to models proposing straightforward temporal changes in sub-Yamato Basin mantle composition, such as replacement of enriched subcontinental upper mantle by injection of depleted asthenospheric mantle into the sub-arc mantle (Tatsumi et al., 1989; Tatsumi et al., 1990; Nohda et al., 1988; Nohda et al., this volume), or replacement of enriched OIB mantle by depleted mantle diapirs (Pouclet and Bellon, this volume).

A goal of the drill sites in the Yamato Basin was to infer the nature of the deeper crust. A principal question was whether the deep crust was oceanic or partially continental in character, the latter virtually required by models of rapid Yamato Basin formation (Takeshita and Yamaji, 1990). While our data and that of Cousens and Allan (this volume) indicate little interaction of the Yamato Basin basaltic melts with continental crust, it does not rule out its presence, either. We note that the recent drilling results from Leg 135 in the Lau Basin (Leg 135 Shipboard Science Party, 1991; Parsons, Hawkins, Allan, et al., in press; Parsons et al., in press) may provide a model for the formation of the Yamato Basin. Leg 135 results indicate that the Lau Basin began to form at least 5.5-6 m.y. ago, with initial extension occurring by diffuse, brittle crustal failure resulting in a "basin-range" seafloor topography. The seafloor spreading now active in the eastern portion of the basin was not organized until about 1-2 m.y. ago, creating a sharp rough-smooth transition in the seafloor fabric. Leg 135 drilling cored basalts and andesites in the narrow (4-10 km wide) north-south basins created during the initial $4 \mathrm{Ma}$ or so of brittle extension, and found that these lavas were transitional in character between MORBs and oceanic arc volcanics, with no relationship of rock composition to either location or age. We speculate that the Yamato Basin with its heterogenous igneous activity may have followed this model, but that seafloor spreading never coherently developed as in the most recent stage of Lau Basin formation; K. Tamaki (pers. comm., 1991) has noted that the Japan Basin, with most of its crust and lithosphere of oceanic character, contains a rough-smooth transition in its seafloor fabric perhaps similar to that newly discovered in the Lau Basin.

\section{ACKNOWLEDGMENTS}

We are indebted to the captains and crew of the SEDCO BP471, and the Operation Superintendents, Lab Officers, and Technical Support staff of the Ocean Drilling Program, Texas A\&M University, for their professionalism and competence in the obtaining of rock core on Legs 127 and 128. We are also grateful to the co-chief and shipboard scientists of Legs 127 and 128 for assistance in the collection and description of the samples. We specifically thank A. Pouclet and S. Scott of Leg 128 for their diligence in trying to match the results of the two legs and for taking such care in the collection of samples for us. Conversations with B. Cousens, S. Yamashita, P. Thy, K. Stewart, K. Tamaki, K. Pisciotto, J. Ingle, K. Suyehiro, A. Pouclet, S. Scott, Y. Tatsumi, T. Fujii, I. Kaneoka, A. Sturz, J. Charvet, S. Sorensen, P. Michael, and the Leg 135 igneous petrologists contributed to the ideas expressed here, although we take sole responsibility for any errors. We thank and acknowledge D. Sims for the production of excellent shipboard XRF data, and thank M. Rhodes and S. Nelson for kindly analyzing samples from units not analyzed on ship. We thank S. Bloomer, J. Pearce, A. Hajash, and an anonymous reviewer for many helpful and constructive comments in their manuscript reviews. J. Mahoney also reviewed the manuscript and helped with the processing of samples, data reduction, and the design of figures. This research was supported by a grant from USSAC, funded by the National Science Foundation.

\section{REFERENCES}

Allan, J. F., Gorton, M. P., Cousens, B. L., and Leg 127 Shipboard Party, 1990. Geochemistry and character of rocks collected by ODP Leg 127 from the Yamato Basin, eastern Japan Sea: implications for back-arc spreading processes. GAC-MAC Annu. Meeting Programs Abstr., Geol. Assoc. Can., 15:A2.

Anders, E., and Ebihara, M., 1982. Solar-system abundances of the elements. Geochim. Cosmochim. Acta, 46:2363-2380.

Arculus, R. J., and Powell, R., 1986. Source component mixing in the regions of arc magma generation. J. Geophys. Res., 91:5913-5926.

Barnes, S.-J., and Gorton, M. P., 1984. Trace element analysis by neutron activation with a low-flux reactor (Slowpoke II): results for international reference rocks. Geostand. Newsl., 8:17-23.

Basaltic Volcanism Study Project, 1981. Basaltic Volcanism on the Terrestrial Planets: New York (Pergamon).

Bienvenu, P., Bougault, H., Joron, J. L., Treuil, M., and Dmitriev, L., 1990. MORB alteration: rare-earth element/non-rare-earth hygromagmaphile element fractionation. Chem. Geol., 82:1-14.

Briqueu, L., Bougault, H., and Joron, L., 1984. Quantification of Nb, Ta, $\mathrm{Ti}$, and $\mathrm{V}$ anomalies in magmas associated with subduction zones: petrographic implications. Earth Planet. Sci. Lett., 68:297-308.

Celaya, M., and McCabe, R., 1987. Kinematic opening of the Sea of Japan and the bending of the Japanese Islands. Geology, 1553-1557.

Dupuy, C., Dostal, J., Marcelot, G., Bougault, H., Joron, J. L., and Treuil, M., 1982. Geochemistry of basalts from central and southern New Hebrides Arc: implication for their source rock composition. Earth Planet. Sci. Lett., 60207-60225.

Einsele, G., 1982. Mechanism of sill intrusion into soft sediment and expulsion of pore water. In Curray, J. R., Moore, D. G., et al., Init. Repts. DSDP, 64 (Pt. 2): Washington (U.S. Govt. Printing Office), 1169-1176.

Erlank, A. J., and Kable, E.J.D., 1976. The significance of incompatible elements in Mid-Atlantic ridge basalts from $45^{\circ} \mathrm{N}$ with particular reference to $\mathrm{Zr} / \mathrm{Nb}$. Contrib. Mineral. Petrol., 54:281-291.

Emewein, M., Pearce, J. A., Bloomer, S. H., Parson, L. M., Murton, B. J., and Johnson, L. E., in press. Geochemistry of Lau Basin volcanic rocks: influence of ridge sedimentation and arc proximity. Contrib. Mineral. Petrol.

Fowler, A. D., 1990. Rock alteration: a review of Pearce diagrams and related techniques. In Russell, J. K., and Stanley, C. R. (Eds.), Theory and Application of Pearce Element Ratios to Geochemical Data Analysis: Vancouver (Clarke Printing), 235-241.

Frey, F. A., Bryan, W. B., and Thompson, G., 1974. Atlantic ocean floor: geochemistry and petrology of basalts from Legs 2 and 3 of the Deep Sea Drilling Project. J. Geophys. Res., 79:5507-5527.

Frey, F. A., Green, D. H., and Roy, S. D., 1978. Integrated models of basalt petrogenesis: a study of quartz tholeiites to olivine melilitites from South Eastern Australia utilizing geochemical and experimental data. J. Petrol., 19:463-513.

Gill, J. B., 1981. Orogenic Andesites and Plate Tectonics: New York (Springer-Verlag).

Green, D. H., Hibberson, W. O., and Jaques, A. L., 1979. Petrogenesis of mid-ocean ridge basalts. In McElhinney, M. W. (Ed.), The Earth: Its Origin, Structure, and Evolution: London (Academic Press), 265-299.

Hajash, A., 1984. Rare earth abundances and distribution patterns in hydrothermally altered basalts: experimental results. Contrib. Mineral. Petrol., $85409-85412$.

Hanson, G. N., 1989, An approach to trace element modeling using a simple igneous system as an example. In Lipin, B. R., and McKay, G. A. (Eds), Reviews of Mineralogy (Vol. 21): Geochemistry and Mineralogy of Rare Earth Elements. Mineral. Soc. Am., 79-97.

Hart, S. R., Erlack, A. J., and Kable, E.J.D., 1974. Sea-floor basalt alteration: some chemical and Sr isotopic effects. Contrib. Mineral. Petrol, 44:219-230.

Hawkins, J. W., Lonsdale, P. F., Macdougall, J. D., Volpe, A. M., 1990. Petrology of the axial ridge of the Mariana Trough backarc spreading center. Earth Planet. Sci. Lett., 100:226-250. 
Hawkins, J. W., and Melchior, J. T., 1985. Petrology of Mariana Trough and Lau Basin basalts. J. Geophys. Res., 90:11431-11468.

Hickey, R. L., Frey, F. A., and Gerlach, D. C., 1986. Multiple sources for basaltic arc rocks from the southern volcanic zone of the Andes $\left(34^{\circ}-41^{\circ} \mathrm{S}\right)$ : trace element and isotopic evidence for contributions from subducted oceanic crust, mantle, and continental crust. J. Geophys. Res., 91:5963-5983.

Hilde, T. W., and Wageman, J. M., 1973. Structure and origin of the Japan Sea. In Coleman, P. J. (Ed.), The Western Pacific: Island Arcs, Marginal Seas, Geochemistry: Perth (Univ. of Western Australia Press), 415-434.

Hirata, N., Tokuyama, H., and Chung, T. W., 1989. An anomolously thick layering of the crust of the Yamato Basin, southeastern Japan Sea: the final stage of back-arc spreading. Tectonophysics, 165:303-314.

Humphris, S. E., and Thompson, G., 1978. Trace element mobility during alteration of oceanic basalts. Geochim. Cosmochim. Acta, 42:127-136.

Hynes, A., 1980. Carbonatization and mobility of TI, Y, and Zr in Ascot Formation metabasalts, SE Quebec. Contrib. Mineral. Petrol., 75:79-87.

Ingle, J. C., Jr., Suyehiro, K., von Breymann, M. T., et al., 1990. Proc. ODP, Init. Repts., 128: College Station, TX (Ocean Drilling Program).

Irving, A. J., 1978. A review of experimental studies of crystal/liquid trace element partitioning. Geochim. Cosmochim. Acta, 42:743-770.

Juteau, T., Noack, Y., Whitechurch, H., and Courtois, C., 1979. Mineralogy and geochemistry of alteration products in Holes 417 A and 417D basement samples (Deep Sea Drilling Project Leg 51). In Donnelly, T., Franchetau, J., Bryan, W., Robinson, P., Flower, M., Salisbury, M., et al., Init. Repts. DSDP, 51, 52, 53: Washington (U.S. Govt. Printing Office), 1273-1279.

Karig, D. E., 1971. Origin and development of marginal basins in the western Pacific. J. Geophys. Res., 76:2542-2561.

1974. Evolution of arc systems in the western Pacific. Annu. Rev. Earth Planet. Sci., 2:51-75.

Lallemand, S., and Jolivet, L., 1986. Japan Sea: a pull-apart basin? Earth Planet. Sci. Lett., 76:375-389.

Laul, J. C., 1978. Neutron activation analysis of geological materials. Atomic Energy Rev., 17:603-695.

Leg 135 Shipboard Scientific Party, 1991. A new view of arc/backarc systems. Geotimes, 36:19-20.

Ludden, J. N., and Thompson, G., 1979. An evaluation of the behaviour of rare earths during the weathering of seafloor basalts. Earth Planet. Sci. Lett., 79:1131-1141.

Ludwig, W. J., Murauchi, S., and Houtz, R. E., 1975. Sediments and structure of the Japan Sea. Geol. Soc. Am. Bull., 86:651-664.

Masuda, A., Nakamura, N., and Tanaka, T., 1973. Fine structures of mutuallynormalized rare-earth patterns of chondrites. Geochim. Cosmochim. Acta, 37:239-248.

Menzies, M. A., Leeman, W. P., Hawkesworth, C. J., 1984. Geochemical and isotopic evidence of continental flood basalts with particular reference to the Snake River Plain Idaho, U.S.A. Philos. Trans. R. Soc. London A, 310:643-660.

Michael, P. J., Chase, R. L., and Allan, J. F., 1989. Petrologic and geologic variations along the Southern Explorer Ridge, northeast Pacific Ocean. J. Geophys. Res., 94:13895-13918.

Nicholls, J., and Russell, J. K., 1990. Pearce element ratios-an overview, example, and bibliography. In Russell, J. K., and Stanley, C. R. (Eds.), Theory and Application of Pearce Element Ratios to Geochemical Data Analysis: Vancouver (Clarke Printing), 11-21.

Nohda, S., Tatsumi, Y., Otofuji, Y., Matsuda, T., and Ishizaka, K., 1988. Asthenopheric injection and back-arc opening: isotopic evidence from NE Japan. Chem. Geol., 68:317-327.

Parsons, L. M., Hawkins, J. W., and Hunter, P. M., in press. Morphotectonics of the Lau Basin seafloor: implications for the opening history of back-arc basins. In Parsons, L., Hawkins, J., Allan, J., et al., Proc. ODP, Init. Repts., 135: College Station TX (Ocean Drilling Program).

Pearce, J. A., 1982. Trace element characteristics of lavas from destructive plate boundaries. In Thorpe, R. S. (Ed.), Andesites: Orogenic Andesites and Related Rocks: Chichester (Wiley), 525-548.

1983. The role of sub-continental lithosphere in magma genesis at destructive plate margins. In Hawkesworth, C. J., and Norry, M. J. (Eds.), Continental Basalts and Mantle Xenoliths: Nantwich (Shiva), 230-249.

Pearce, J. A., Alabaster, T., Shelton, A. W., and Searle, M. P., 1981. The Oman ophiolite as a Cretaceous arc-basin complex: evidence and implications. Philos. Trans. R. Soc. London A, A300:299-317.

Pearce, T. H., 1968. A contribution to the theory of variation diagrams. Contrib. Mineral. Petrol., 19:142-157.
Perfit, M. R., Gust, D. A., Bence, A. E., Arculus, R. J., and Taylor, S. R., 1980. Chemical characteristics of island-arc basalts: implications for mantle sources. Chem. Geol., 30:227-256.

Rhodes, J. M., 1988. Geochemistry of the 1984 Mauna Loa eruption: implications for magma storage and supply. J. Geophys. Res., 93:4453-4466.

Russell, J. K., and Nicholls, J., 1988. Analysis of petrographic hypotheses with Pearce element ratios. Contrib. Mineral. Petrol., 99:25-35.

Saunders, A. D., Fornari, D. J., Joron, J.-L., Tarney, J., and Treuil, M., 1982. Geochemistry of basic igneous rocks, Gulf of California, Deep Sea Drilling Project Leg 64. In Curray, J. R., Moore, D. G., et al., Init. Repts. DSDP. 64: Washington (U.S. Govt. Printing Office), 595-642.

Saunders, A. D., and Tarney, J., 1984. Geochemical characteristics of basaltic volcanism within back-arc basins. In Kokelaar, B. P., and Howells, M. F. (Eds.), Marginal Basin Geology: London (Blackwell Sci.), 59-76.

Schilling, J.-G., 1975. Rare-earth variations across "normal segments" of the Reykjanes Ridge, $60^{\circ}-53^{\circ} \mathrm{N}$, Mid-Atlantic Ridge, $29^{\circ} \mathrm{S}$, and East Pacific Rise, $2^{\circ}-19^{\circ} \mathrm{S}$, and evidence on the composition of the underlying low-velocity layer. J. Geophys. Res., 80:1459-1473.

Seyfried, W. E., Jr., 1987. Experimental and theoretical constraints on hydrothermal alteration processes at mid-ocean ridges. Annu. Rev. Earth Planet. Sci., 15:317-335.

Shannon, R. D., 1976. Revised effective ionic radii and systematic studies of interatomic distances in halides and chalcogenides. Acta Cryst., A32:751-767.

Shimazu, M., Yoon, M., and Tateishi, M., 1990. Tectonics and volcanism in the Sado-Pohang Belt from 20 to $14 \mathrm{Ma}$ and opening of the Yamato Basin of the Japan Sea. Tectonophysics, 181:321-330.

Stix, J., and Gorton, M. P., 1990. Changes in silicic melt structure between the two Bandelier caldera-forming eruptions, New Mexico, USA: evidence from zirconium and light rare earth elements. J. Petrol., 31:1261-1283.

Sun, S. S., Nesbitt, R. W., and Sharaskin, A. Y., 1979. Geochemical characteristics of mid-ocean ridge basalts. Earth Planet. Sci. Lett., 44:119-138.

Takahashi, E., and Kushiro, I., 1983. Melting of a dry peridotite at high pressures and basalt magma genesis. Am. Mineral., 68:859-879.

Takeshita, T., and Yamaji, A., 1990. Acceleration of continental rifting due to a thermomechanical instability. Tectonophysics, 181:307-320.

Tamaki, K., 1985. Two modes of back-arc spreading. Geology, 13475-13478.

Tamaki, K., Pisciotto, K., Allan, J., et al., 1990. Proc. ODP, Init. Repts., 127: College Station, TX (Ocean Drilling Program).

Tatsumi, Y., Hamilton, D. L., and Nesbitt, R. W., 1986. Chemical characteristics of fluid phase released from a subducted lithosphere and origin of arc magmas: evidence from high-pressure experiments and natural rocks. J. Volcanol. Geotherm. Res., 29:293-309.

Tatsumi, Y., Maruyama, S., and Nohda, S., 1990. Mechanism of back-arc opening in the Japan Sea: role of asthenospheric inection. Tectonophysics, 181:299-306.

Tatsumi, Y., Otofuji, Y.-I., Matsuda, T., and Nohda, S., 1989. Opening of the Japan back-arc basin by asthenopheric projection. Tectonophysics, 166:317-329.

Tsuchiya, N., 1988. Distribution and chemical composition of the middle Miocene basaltic rocks in Akita-Yamagata oil fields of Northeastern Japan. Chishitsugaku YZasshi, 94:591-608.

, 1989. Submarine basalt volcanism of Miocene Aosawa Formation in the Akita-Yamagata oil field basin, back-arc region of Northeast Japan. Chishitsugaku Ronshu, 32:399-408.

1990. Middle Miocene back-arc rift magmatism of basalt in the NE Japan arc. Bull. Geol. Soc. Jpn., 41:473-505.

Volpe, A. M., Macdougall, J. D., and Hawkins, J. W., 1987. Mariana Trough Basalts (MTB): trace element and Sr-Nd isotopic evidence for between MORB-like and arc-like melts. Earth Planet. Sci. Lett., 82:241-254.

Wilson, M., 1989. Igneous Petrogenesis: London (Unwin Hyman),

Wood, D. A., Joron, J.-L., and Treuil, M., 1979. A re-appraisal of the use of trace elements to classify and discriminate between magma series erupted in different tectonic settings. Earth Planet. Sci. Lett., 45:326-336.

Zindler, A., and Hart, S. R., 1986. Chemical geodynamics. Annu. Rev. Earth Planet. Sci., 14:493-571.

Date of initial receipt: 7 May 1991

Date of acceptance: 10 February 1992

Ms 127/128B-208 